\title{
Automating Software Evolution: Towards Using Constraints with Action for Model Evolution
}

By

Shahid Alam, B. Eng., M. Sc.

\author{
A Thesis submitted to the \\ Faculty of Graduate Studies and Research \\ in partial fulfillment of the \\ requirements for the degree of
}

\author{
Master of Applied Science \\ in \\ Electrical Engineering
}
Ottawa-Carleton Institute for Electrical and Computer Engineering Department of Systems and Computer Engineering Carleton University, Ottawa, Ontario
(C) Copyright Shahid Alam 2007




$\begin{array}{ll}\begin{array}{l}\text { Library and } \\ \text { Archives Canada }\end{array} & \begin{array}{l}\text { Bibliothèque et } \\ \text { Archives Canada }\end{array} \\ \begin{array}{l}\text { Published Heritage } \\ \text { Branch }\end{array} & \begin{array}{l}\text { Direction du } \\ \text { Patrimoine de l'édition }\end{array} \\ \begin{array}{l}\text { 395 Wellington Street } \\ \text { Ottawa ON K1A ON4 }\end{array} & \begin{array}{l}\text { 395, rue Wellington } \\ \text { Ottawa ON K1A ON4 } \\ \text { Canada }\end{array}\end{array}$

Your file Votre référence ISBN: 978-0-494-33635-9 Our file Notre référence ISBN: 978-0-494-33635-9

NOTICE:

The author has granted a nonexclusive license allowing Library and Archives Canada to reproduce, publish, archive, preserve, conserve, communicate to the public by telecommunication or on the Internet, loan, distribute and sell theses worldwide, for commercial or noncommercial purposes, in microform, paper, electronic and/or any other formats.

The author retains copyright ownership and moral rights in this thesis. Neither the thesis nor substantial extracts from it may be printed or otherwise reproduced without the author's permission.
AVIS:

L'auteur a accordé une licence non exclusive permettant à la Bibliothèque et Archives Canada de reproduire, publier, archiver, sauvegarder, conserver, transmettre au public par télécommunication ou par l'Internet, prêter, distribuer et vendre des thèses partout dans le monde, à des fins commerciales ou autres, sur support microforme, papier, électronique et/ou autres formats.

L'auteur conserve la propriété du droit d'auteur et des droits moraux qui protège cette thèse. $\mathrm{Ni}$ la thèse ni des extraits substantiels de celle-ci ne doivent être imprimés ou autrement reproduits sans son autorisation.
In compliance with the Canadian

Privacy Act some supporting forms may have been removed from this thesis.

While these forms may be included in the document page count, their removal does not represent any loss of content from the thesis.
Conformément à la loi canadienne sur la protection de la vie privée, quelques formulaires secondaires ont été enlevés de cette thèse.

Bien que ces formulaires aient inclus dans la pagination, il n'y aura aucun contenu manquant.

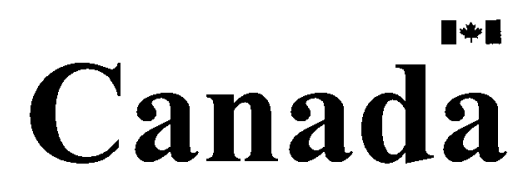




\section{Abstract}

In this thesis we examine two issues and propose solutions to resolve them. The first is the automation of model evolution and the second is the support of software evolution in modeling languages. We extend Object Constraint Language with actions and define a new language CAL (Constraints with Action Language), which gives a user the ability to use constraints with actions on models. We have added a data type, directed acyclic graph (DAG) to CAL to automate model evolution and optimize impact analysis, and to make CAL more accessible to non-programmers. The DAG has been annotated (ADAG) with dependency weights and a labeling scheme has been improved in size and used to optimize basic ADAG operations. CAL contains a small set of constructs, but is powerful enough to be used efficiently for typical software evolution management operations. A prototype tool VCAL, for dependency analysis of UML Class Diagrams is presented. 


\section{Acknowledgements}

I would like to acknowledge the contributions of Dr. Samuel A. Ajila to the writing of this thesis in the form of content, resources, commitment and support. I would specially like to mention his encouragement and guidance, for writing research papers and becoming a part of the software engineering research community.

Numerous other people deserve to be mentioned for their parts in my Master's thesis. I would like to thank Professor Samuel A. Ajila and Professor Halim Yanikomeroglu and other staff members of the department for their advice and support during my master's program. The Graduate Communication Workshop by Professor Logie, and Kate Parsons of the Academic Writing Centre provided guidance and feedback in writing this thesis.

Special thanks go to my family: my parents, wife and four kids for their understanding, support and help, which enabled me to accomplish this. 


\section{Acronyms}

$\mathrm{ADAG}=$ Annotated Directed Acyclic Graph

$\mathrm{ADP}=$ Acyclic Dependency Principle

$\mathrm{AOP}=$ Aspect Oriented Programming

AS $=$ Action Semantics

$\mathrm{ASL}=$ Action Specification Language

AST $=$ Abstract Syntax Tree

$\mathrm{AWT}=$ Abstract Window Toolkit

BFS $=$ Breath First Search

$\mathrm{CAL}=$ Constraints with Action Language

CASE $=$ Computer Aided Software Engineering

$\mathrm{CFG}=$ Context Free Grammar

DFS $=$ Depth First Search

$\mathrm{EBNF}=$ Extended Backus-Naur Form

ECL $=$ Embedded Constraint Language

GUI $=$ Graphical User Interface

MDA $=$ Model Driven Architecture

MDSE = Model Driven Software Engineering

OAL $=$ Object Action Language

$\mathrm{OCL}=$ Object Constraint Language

OMG = Object Management Group 
$\mathrm{PAL}=$ Platform Independent Action Language

PIM = Platform Independent Model

PSM = Platform Specific Model

RFP $=$ Request for Proposal

SWT $=$ Software Widget Toolkit

TLA $=$ Temporal Logic of Action

$\mathrm{UML}=$ Unified Modeling Language

$\mathrm{VCAL}=$ Visual $\mathrm{CAL}$ 


\section{Contents}

ABSTRACT III

ACKNOWLEDGEMENTS IV

$\begin{array}{lll}\text { ACRONYMS } & \text { V }\end{array}$

INTRODUCTION

1.1 Motivation for CAL 2

$\begin{array}{ll}\text { 1.2 Problem statement } & 3\end{array}$

$\begin{array}{ll}1.3 \text { Goals } & 5\end{array}$

1.4 Approach used

$\begin{array}{ll}1.5 \text { Research contributions } & 9\end{array}$

$\begin{array}{ll}\text { 1.6 Organization of this thesis } & 11\end{array}$

BACKGROUND AND MODEL EVOLUTION 12

2.1 Software evolution $\quad 12$

2.1.1 Issues in software evolution $\quad 13$

$\begin{array}{ll}\text { 2.1.1.1 Raising the level of abstraction } & 13\end{array}$

$\begin{array}{ll}\text { 2.1.1.2 What, how and why? } & 13\end{array}$

2.2 Dependency relationships between UML model elements 14

2.3 Formal languages $\quad 14$

$\begin{array}{ll}\text { 2.3.1 Specification of formal languages } & 17\end{array}$

2.3.2 Left recursion removal in LL(k) grammar $\quad 18$

$\begin{array}{ll}\text { 2.3.3 Left factoring } & 18\end{array}$

$\begin{array}{ll}2.4 \text { Executable UML } & 19\end{array}$

2.5 OCL (Object Constraint Language) $\quad 20$

$\begin{array}{ll}2.5 .1 \text { OCL collections } & 22\end{array}$

$\begin{array}{ll}2.5 .2 \text { OCL navigation } & 22\end{array}$

2.5.3 OCL standard library $\quad 22$ 
2.6 TLA (Temporal Logic of Actions)

2.6.1 An algorithm language (pluscal) based on TLA 25

2.7 Related work in model evolution

$\begin{array}{ll}2.7 .1 \text { Evolution contracts } & 29\end{array}$

2.7.2 Refactoring $\quad 30$

2.7.3 Model evolution and impact analysis 31

2.7.4 Recent languages for model transformation 33

$\begin{array}{ll}2.7 .5 \text { Comparison } & 35\end{array}$

2.7.6 Impact analysis support in commercial UML tools 38

$\begin{array}{ll}\text { CAL } & 41\end{array}$

3.1 CAL grammar $\quad \mathbf{4 2}$

3.1.1 Production rules added for CAL $\quad 43$

3.2 CAL library

3.2.1 DagType 45

$\begin{array}{ll}3.2 .2 \mathrm{Dag} & 46\end{array}$

$\begin{array}{ll}3.2 .3 \text { isdag } & 47\end{array}$

$\begin{array}{ll}3.2 .4 \text { size } & 47\end{array}$

$\begin{array}{ll}3.2 .5 \text { isempty } & 47\end{array}$

$\begin{array}{ll}3.2 .6 \text { flatten } & 47\end{array}$

3.2 .7 includes $\quad 48$

3.2 .8 excludes $\quad 48$

3.2.9 depend $\quad 48$

3.2.10 descendants $\quad 49$

3.2.11 view 50

3.3 CAL actions $\quad 50$

3.3.1 create 51

3.3.2 delete 51

3.3.3 link 52

3.3.4 unlink $\quad 52$

3.3.5 addattr 53

3.4 Usage $\quad 53$

3.5 CAL and other action languages $\quad 55$

DAG FOR DEPENDENCY ANALYSIS

4.1 DAG Labeling

4.1.1 Labeling scheme 66

4.1.2 Size of the label $\quad 68$

4.2 Labeling and dependency analysis algorithms 69

viii 
$\begin{array}{ll}4.3 \text { Updates } & 73\end{array}$

$\begin{array}{ll}\text { 4.4 Related work } & 74\end{array}$

$\begin{array}{ll}\text { CAL IMPLEMENTATION } & 79\end{array}$

5.1 Rules for translating a UML model to the CAL data structure 79

$\begin{array}{lr}\text { 5.2 CAL model } & 81\end{array}$

$\begin{array}{ll}\text { 5.2.1 ADAG operations } & 83\end{array}$

5.2.1.1 Includes $\quad 84$

$\begin{array}{ll}\text { 5.2.1.2 Excludes } & 84\end{array}$

$\begin{array}{ll}\text { 5.2.1.3 Depend } & 84\end{array}$

$\begin{array}{ll}\text { 5.2.1.4 Descendants } & 87\end{array}$

$\begin{array}{ll}\text { 5.2.2 CAL actions } & 88\end{array}$

$\begin{array}{ll}\text { 5.2.2.1 Create } & 88\end{array}$

$\begin{array}{ll}5.2 .2 .2 \text { Relabel } & 89\end{array}$

$\begin{array}{lr}\text { 5.2.2.3 Setweight } & 90\end{array}$

5.3 Formal verification using TLC $\quad 90$

$\begin{array}{ll}\text { CAL PROTOTYPES } & 95\end{array}$

$\begin{array}{ll}\text { 6.1 CAL parser } & 95\end{array}$

6.2 Implementation details $\quad 96$

6.2.1 Class Parser $\quad 97$

6.2.2 Class OclStmt $\quad 98$

6.2.3 Class Lexer $\quad 98$

6.2.4 Class AST $\quad 98$

$\begin{array}{lr}\text { 6.2.4.1 Data structure for storing the AST } & 98\end{array}$

$\begin{array}{lr}\text { 6.2.5 Class Symtable } & 101\end{array}$

$\begin{array}{ll}\text { 6.2.5.1 Collision resolution } & 101\end{array}$

$\begin{array}{ll}\text { 6.2.5.1.1 Chaining } & 101\end{array}$

$\begin{array}{ll}\text { 6.2.5.1.2 Open addressing } & 102\end{array}$

$\begin{array}{ll}\text { 6.2.6 Class LG } & 104\end{array}$

$\begin{array}{ll}\text { 6.2.7 Class List } & 104\end{array}$

6.3 VCAL (visual CAL) $\quad 105$

6.3.1 CAL parser and CAL file $\quad 106$

$\begin{array}{ll}\text { 6.3.2 ProcessXMI } & 106\end{array}$

6.3.3 ADAG (annotated DAG) 107

$\begin{array}{ll}\text { 6.3.4 CAL Interpreter } & 107\end{array}$

$\begin{array}{ll}\text { 6.3.5 GUI (graphical user interface) } & 107\end{array}$

$\begin{array}{ll}\text { 6.3.6 Running VCAL } & 109\end{array}$ 
7.1 Limitations

7.2 Future research

APPENDIX 'B' CAL PARSER'S FINITE STATE MACHINE FOR LEXICAL ANALYSIS

APPENDIX 'C' CAL PARSER'S SYNTAX AND PARSING TREE

APPENDIX 'E' CAL SPECIFICATIONS (ASCII VERSION USING PLUSCAL, TRANSLATION NOT SHOWN) IN TLA+ WITH CONFIGURATION FILE AND TLC OUTPUT 


\section{List of Tables}

2.1 Stereotypes for dependency in UML 2.0

2.2 Dependency relationships between UML model elements 16

2.3 Current OCL tools $\quad 40$

3.1 Comparison of CAL with other action languages 59

5.1 Characteristics and coupling of different relationships in UML 81

6.1 Comparison of LL and LR parsing 97

$\begin{array}{lll}\text { 6.2 Chaining versus open addressing } & 103\end{array}$

$\begin{array}{lll}\text { 6.3 Test matrix for the case study } & 112\end{array}$ 


\section{List of Figures}

1.1 Overview of the solution proposed in this thesis 10

$\begin{array}{lll}1.2 & \text { Overview of the CAL model verification } & 10\end{array}$

2.1 Simple Clock Example from the TLA book [50] 26

$\begin{array}{lll}2.2 & \text { Euclid Algorithm in pluscal } & 28\end{array}$

$3.1 \quad$ Production rules for CAL $\quad 44$

3.2 Sample CAL file $\quad 54$

$\begin{array}{lll}4.1 & \text { Sample DAG } & 65\end{array}$

4.2 Equations for computing the label of a vertex in the DAG 67

$\begin{array}{lll}4.3 & \text { Labeled DAG } & 68\end{array}$

$\begin{array}{lll}4.4 & \text { Algorithms to label a node in the DAG } & 70\end{array}$

4.5 Algorithm to find descendants of a node in the DAG 71

4.6 Algorithm to find reachability of two nodes in the DAG 72

4.7 Algorithms to remove the link of a node from the DAG, and check if a node is $\begin{array}{ll}\text { present or not in the DAG } & 73\end{array}$

4.8 Algorithm to set the parent of a node in the DAG $\quad 74$

4.9 Algorithms to add and delete a node in the DAG $\quad 75$

$\begin{array}{lll}4.10 & \text { Labeled DAG with node R Added } & 76\end{array}$

4.11 Labeled DAG with node K Removed 77

5.1 Global variables (data) and define block $\quad 83$

$\begin{array}{lll}\text { 5.2 } & \text { ADAG operation includes } & 84\end{array}$

$\begin{array}{lll}\text { 5.3 ADAG operation excludes } & 84\end{array}$ 
5.4 ADAG operation CheckDependency $\quad 85$

$\begin{array}{lll}\text { 5.5 ADAG operation depend } & 86\end{array}$

$\begin{array}{lll}\text { 5.6 } & \text { ADAG operation descendants } & 87\end{array}$

$\begin{array}{lll}\text { 5.7 } & \text { CAL action create } & 89\end{array}$

$\begin{array}{lll}\text { 5.8 } & \text { CAL sub-action relabel } & 90\end{array}$

$\begin{array}{lll}\text { 5.9 CAL sub-action setweight } & 91\end{array}$

5.10 Part of the main block of CAL specifications, and the CAL data structure (ADAG) before and after annotations $\quad 92$

5.11 Part of the TLC output 93

$\begin{array}{lll}\text { 6.1 Data structure for storing AST } & 99\end{array}$

6.2 XML output generated by the Class AST 100

$\begin{array}{lll}6.3 & \text { Collision resolution by chaining } & 102\end{array}$

$\begin{array}{lll}6.4 & \text { Collision resolution by open addressing } 103\end{array}$

6.5 Linked List with pointer to the data 105

$\begin{array}{lll}6.2 & \text { Overview of the tool (VCAL) } & 105\end{array}$

$\begin{array}{lll}6.7 & \text { VCAL GUI } & 108\end{array}$

6.8 UML Class Diagram for the CAL parser in XMI 110

6.9 CAL files, with different CAL actions and operations 113

6.10 VCAL GUI, showing DAG after executing different CAL actions and operations shown in Figure 6.9 114 


\section{Chapter 1}

\section{Introduction}

Software evolution has become a consistent and major part of the software life cycle. More time is spent on changing than developing the software. Of all the software processes evolution is the most expensive. There is a need to evolve the system from the beginning. To achieve this we want to integrate software evolution in the design or the model of the software system. Since the advent of model driven software engineering (MDSE) it has become more necessary to develop techniques and tools for model evolution. Due to the lack of support of software evolution management in modeling languages and tools, it is highly desirable to integrate such support in these modeling languages and tools. None of the previous efforts, either commercial $[24,25,26,33,34$, $35]$ or academic $[12,14,15,19,20,22,28]$, support automation of software evolution in a modeling language. This thesis proposes a novel approach for the automated management of software evolution in models. The basic purpose of this approach is to show that a modeling language can be used to automate software evolution in models.

In this thesis, we extend OCL (Object Constraint Language) and define a new language CAL (Constraints with Action Language). We have added 5 actions, 10 operations and 1 data type to CAL. OCL is a query modeling language [2] used for the models specified in a Unified Modeling Language (UML) [5] and can also be used for other models. OCL can be used to define constraints (rules) for impact analysis [22], but 
the use of constraints is not the optimal way to perform impact analysis, and moreover OCL cannot be used to make changes to a model. CAL gives the user the ability to use constraints with actions on models. Because of the characteristics (transitive and nonreflexive) of each dependency relationship between UML Class Model elements, a new set of Collection directed acyclic graph (DAG) has been added to the CAL library for automatically generating these dependency relationships. The DAG has been annotated for dependency analysis, with dependency weights and an improved labeling scheme has been proposed and used to optimize basic DAG operations (also called CAL operations). We call this DAG an annotated DAG (ADAG).

CAL contains a small set of constructs, but is powerful enough to be used efficiently for typical software evolution management operations like impact analysis, correction, improvement and enhancement of models. CAL can be extended and used, as an action language, or in model compilers for incremental recompilation and retransformation of models, in model driven architecture (MDA) $[11,47]$. Since CAL has its own well defined data structure and type system, it is independent of other modeling tools API's, data structure and type system. This makes CAL highly interoperable, portable and flexible.

\subsection{Motivation for CAL}

There are two important concepts in software engineering, use of models and automation in software engineering, on which this thesis is based. Separating the model of the software system from its implementation allows us to integrate software evolution in the design of the software system. Software models are easier to maintain, evolve and understand. Empirical investigation shows that even experienced software professionals 
predict incomplete sets of software changes [45]. One of the challenges [44] facing software evolution is to raise the level of abstraction and is called model evolution.

Introduction of MDA by object management group (OMG) has opened up new opportunities in model evolution. MDA introduces the concept of executable models, to raise the level of abstraction. Executable models are computationally complete models and therefore can be executed $[9,16]$. The implementation follows the model and not vice versa and hence we only need to change the model. MDA is an important software design approach towards MDSE, creating new opportunities for model evolution. There are two issues that our work examines and resolves. One of them is the support of software evolution in modeling languages. There are many computer aided software engineering (CASE) tools including commercial, open source and academic that are being used in MDSE. Automation of the software development life cycle is at the heart of these CASE tools. The other opportunity is the automation of model evolution.

\subsection{Problem statement}

Impact analysis is defined as identifying the potential consequences of a change, or estimating what needs to be modified to accomplish a change [7] and is one of the methods used in managing software evolution. Although it is easier to collect the necessary information for impact analysis at detailed design level than at the source code level, still impact analysis of models faces problems. Some of these problems, which this thesis examines, are listed below:

\section{- Lack of support of management of software evolution in modeling languages}

UML is a well-established industrial standard for modeling software systems and is becoming more mature as time passes. It has notations, symbols and stereotypes to 
define dependency relationships, but there is no modeling technique for automated software evolution management. OCL or other languages $[24,25,26]$ do not directly provide support for model evolution.

\section{- Automation of the management of software evolution in models}

There are several reasons for change and it is not easy to identify the impact of change in a software system [7]. Dependency relationships, whether direct or indirect between UML model elements are numerous. To find them by hand is not practical as it involves lot of labor and hence is prone to errors. It is highly desirable to develop techniques to automate this process to reduce errors, time and money especially for large and complex systems. After identifying these dependency relationships, how can we automatically perform typical software maintenance operations like correction, improvement and enhancement to the model? There are various efforts at detecting, tracing and managing evolution but very few $[14,18,22]$ deal with automating this process and none cover all the aspects mentioned above.

\section{- Lack of support of automated management of software evolution in tools}

Impact analysis of the model should be part of a good CASE tool. We not only need support of tools but we need them to automate this process with as little input from the user as possible. Currently there is lack of support of impact analysis in CASE tools. Very few CASE tools provide this facility either by themselves through a set of procedural application programming interfaces (API's) $[32,33,34]$ or by third party plug-ins such as Gateway [35] in Rhapsody. If it is provided then it is highly dependent on the CASE tool and requires great amount of effort from the user, so this indirect support is not portable, flexible and automatic. 


\section{- Incremental recompilation and retransformation of models}

MDA uses model transformation language to transform model from platform independent model (PIM) to platform specific model (PSM). Executable UML [9, 16] takes the concept of MDA further. It removes the PSM from MDA and directly generates code that can execute. How can change be propagated automatically to the transformed model or to the generated code, so that when the software modeler makes a change to the model, the model compiler is able to detect which elements of the model have been modified and then use an impact algorithm to determine what minimal elements, need to be recompiled or retransformed, and in what order?

\subsection{Goals}

We desire a practical solution that gives us the ability to automate the process of generating the dependency relationships among the model elements for impact analysis. Besides being automatic it should be visual, portable, efficient, flexible and provide ease of use. Following is a description of these characteristics in the context of this thesis:

\section{- Portability}

The solution should be portable to other CASE tools, which means it should be easily integrated into other CASE tools and makes it more reusable. It should be independent of any domain (like hardware, embedded systems etc).

\section{- Flexibility}

It is highly desirable for a software evolution system to do updates. It should be able to update the changes made in the model. Update here, means updating the changes in the model without going back to the original model. In this way, there is no need to compare two models or to keep an extra model in the working 
memory before making a change. Besides updates the solution should be scalable to accommodate future extensions.

\section{- Efficiency}

The queries for finding dependencies among model elements should take less time. In an ideal case the time should be constant i.e. $O(I)$ time to complete. Since flexibility impacts on efficiency, it needs to compensate for that while updating.

\section{- Ease of use}

A CASE tool or any tool is judged by its ease of use. It should require least amount of input from the user for generating impact analysis results. The user should be able to interact with the results.

\section{- Visualization}

Visual or graphical presentation of impact analysis of software change is highly desirable in a CASE tool. Graphical abstraction helps us focus on the relationships among elements of the model that are being maintained.

Besides these characteristics we would like to see if the solution or part of the solution has a mathematical model and uses well defined standard notations to describe its rules.

\subsection{Approach used}

In this section we define an approach that is used in solving the problems and achieving the goals mentioned above. OCL is basically used as a query language for UML. Expressions such as constraints and rules in OCL can be used to identify changes [22], but cannot be used to change the state of the system [2]. Therefore, OCL alone cannot be used for making changes to the model. Action semantics [27] is another OMG 
standard and is now part of UML 2.0 that is specifically defined for this purpose. We have extended OCL with actions and defined a new language CAL. OMG does not define any syntax or semantics for the actions, so we have formally defined the syntax and semantics of CAL actions. A new collection library DAG has been added to automatically generate dependency relationships, which can be queried and elements can be added or deleted using different library functions. Dependency weights have been added to the DAG for dependency analysis. These weights are based on the coupling of different dependency relationships between UML Class Model elements. This gives us a powerful way of querying for dependencies and changing and manipulating UML Class Model elements to achieve automated software evolution management of UML Class Models. CAL is well defined with a grammar in EBNF [1] and an efficient LL(1) parser implemented in $\mathrm{C}++$ for this thesis. CAL is based on OCL and action semantics, which make this solution highly portable and flexible as defined above.

An understanding of dependency relationships between model elements is necessary for efficient impact analysis of models. First we distinguish all the dependency relationships between UML Class Model elements. Then we store these dependencies in a dependency graph. Each vertex represents a UML Class Model element and each edge represents a dependency relationship between the elements. Each vertex is assigned a weight as described above for dependency analysis. The characteristics of each dependency relationship are transitive and non-reflexive. We also assume a good object oriented design based on the Acyclic Dependencies Principle (ADP) [46] i.e. no cycles between model elements. Therefore, the dependency graph by definition is a DAG. The rules of translation from a UML Class Model to the DAG have been formally specified 
for better comprehension. As mentioned above one of the CAL libraries gives user the ability to query, add or delete elements from the DAG. In this way we can perform all the tasks of impact analysis [7] using the DAG. As executable UML comprises the Class Model, the State Machines for the classes and the state procedures, so we restrict our dependency relationships to Class Diagrams for the prototype tool VCAL (Visual CAL), which is implemented for this thesis, but the approach can be used for other UML elements, which can be developed independently of other elements and satisfies ADP.

An improved labeling scheme is proposed for labeling the DAG to achieve efficient query operations for finding reachability between two elements and descendants of an element and add and delete operations. Reachability means that the two elements are dependent and descendants of an element are a set of elements that depend on this element. Both of these can be used to analyze the impact of changing the element. Each element's dependency weight can be calculated by adding all its descendant's weight, which further helps in analyzing the dependencies and the impact of change. The prototype tool VCAL implemented for this thesis checks for reachability between two or more than two elements and descendants of an element.

After adding weights and labeling the DAG we call it an annotated DAG (ADAG). For visualization of the $\mathrm{ADAG}$ we use Java graphics visualization and layout library JGraph [48], which was easy to implement for a research based prototype solution. But for commercial purposes we recommend $\mathrm{C}++$ with OpenGL [49], which is being used in commercial CASE tools for simulations like Simulink and Matlab. Standard widget toolkit (SWT) [54] has been used for design and development of the GUI. It is a Java class library that allows the user to create native user interfaces. It is 
designed to provide efficient, portable access to the underlying facilities of the operating system on which it is implemented. SWT implementation uses Java native interface (JNI) [55] to accomplish this. Figure 1.1 shows an overview diagram in UML of the components of the prototype tool VCAL implemented as part of the solution. We have tested and verified this tool using the CAL parser UML class diagram as an example case study.

\subsection{Research contributions}

Following are the research contributions of this thesis:

$\checkmark$ There are two issues that our work examines and resolves. One of them is the support of software evolution in modeling languages. The other issue is the automation of model evolution.

$\checkmark$ A solution is provided in the form of a modeling language CAL (Constraints with Action Language). An overview of the solution is shown in Figure 1.1. CAL gives a user (modeler) the ability to use constraints with actions on models, and provides the user with tools to automate model evolution.

$\checkmark$ A new set of Collection DAG has been added to the CAL library. The DAG has been annotated (ADAG) with dependency weights for dependency analysis, and a labeling scheme has been improved and used to optimize basic ADAG operations (also called CAL operations).

$\checkmark$ We have also formally defined rules to translate a UML Class Model to the CAL data structure (ADAG).

$\checkmark$ The CAL model, including all the CAL operations and their libraries and one of the CAL actions, create, and its sub-actions, relabel and setweight, have been 
formally specified using pluscal [53], which is based on Temporal Logic of Actions+ (TLA+) [50], and is verified using TLC [50], a TLA+ model checker (see Figure 1.2).

$\checkmark$ A prototype tool, VCAL, has been developed to show how CAL can be used to automatically generate dependency relationships among model elements of UML Class Diagrams to perform typical software evolution management operations (like impact analysis, correction, improvement and enhancement) with as little input from the user as possible. VCAL also visualizes and applies basic filters to the ADAG.

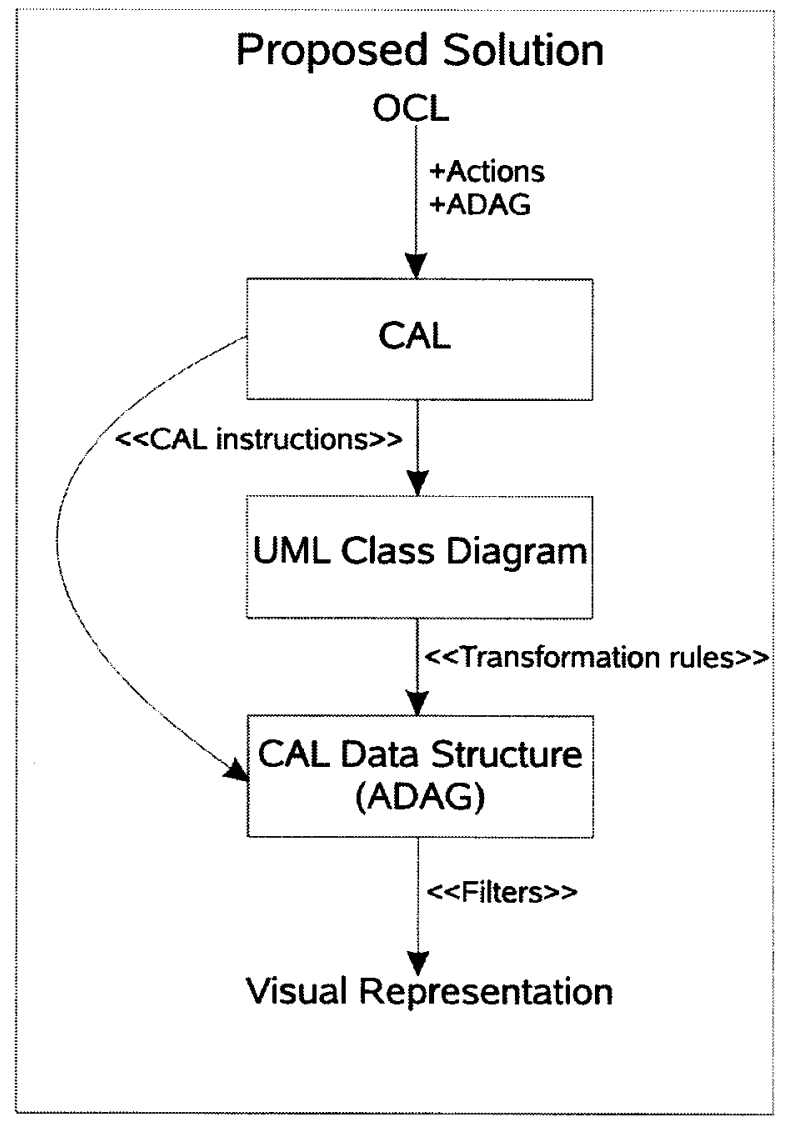

Figure 1.1 Overview of the solution proposed in this thesis
Specification and Verification of CAL

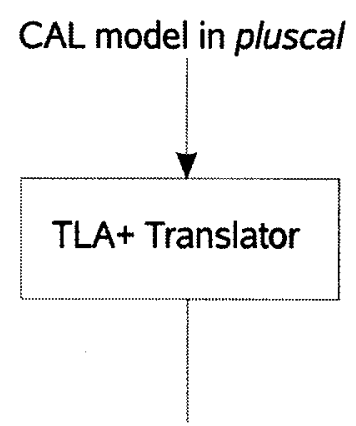

CAL model in TLA+

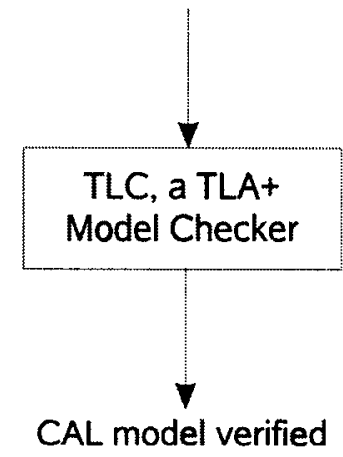

Figure 1.2 Overview of the CAL model verification 


\subsection{Organization of this thesis}

This chapter gives an introduction, discusses motivation and briefly describes specific issues that this thesis examines. It sets up some goals and then provides an approach that is used to solve the issues and achieve these goals.

Chapter 2 provides a background study, which aids in understanding CAL, its formal specifications, parser and the techniques used in implementing CAL, and a literature review on model evolution. At the end of chapter 2 we also compare two of the recent approaches with CAL.

Chapter 3 describes CAL in detail, including its actions, operations and data structure, and also compares it with other action languages for software models.

Chapter 4 discusses in detail the CAL data structure (ADAG) and proposes an improved labeling scheme for the ADAG for dependency analysis.

In chapter 5 we formally specify rules for translating a UML Class Model to the CAL data structure, and all the CAL operations, and one of the CAL actions, create, and its sub-actions, relabel and setweight using TLAt. Chapter 5 also describes the testing and verification of these specifications using TLC.

Chapter 6 describes the CAL parser and its implementation in detail. It also briefly explains and tests the prototype tool VCAL using a case study, which is the UML model of the CAL parser itself.

Chapter 7 concludes the thesis and highlights research contributions of this thesis and future research possible with CAL. 


\section{Chapter 2}

\section{Background and Model Evolution}

This Chapter is divided in two parts. The first part provides background on software evolution, dependency relationships between Unified Modeling Language (UML) model elements, formal languages $[3,6]$, executable UML $[9,16]$, object constraint language (OCL) [2] and temporal logic of actions (TLA) [50], which aids in understanding constraints with action language (CAL), its formal specifications, parser and the techniques used. The second part describes the state of the art in model evolution, starting from evolution contracts [28] by Tom Mens and Theo D'Hondt in the year 2000, to the most recent efforts on model evolution and impact analysis. At the end a comparison is made between two of these efforts with CAL.

\subsection{Software evolution}

Biological species evolve for various reasons and one of them is to better cope with their environments. Similarly there are various reasons why software evolves and needs maintenance. We can say that software evolution is part of software maintenance and in this thesis we do not differentiate between software evolution and software maintenance. Of all the software development processes evolution is the most expensive. Hence, it needs more research and practice. The Research Institue in Software Evolution (RISE) [61] formally defines software evolution as the following:

"The set of activities, both technical and managerial, that ensures that software 
continues to meet organisational and business objectives in a cost effective way".

\subsubsection{Issues in software evolution}

The European Research Consortium for Informatics and Mathematics (ERCIM) and European Science Foundation (ESF) jointly organized a workshop, Challenges in Software Evolution (ChaSE 2005) [44], in April 2005. The aim of the workshop was to identify substantial obstacles to software evolution research and practice and to propose and discuss challenges in software evolution. The top 5 challenges in the list composed by this workshop are: (1) theory of software evolution, (2) raising the level of abstraction, (3) tool complexity, (4) language support and (5) process support. We discuss challenge number (2) below:

\subsubsection{Raising the level of abstraction}

Software is more than the source code. Models and metamodels play a key role in software evolution [52]. Software itself does not evolve but our understanding about it changes. Hence, the models that define the structure of the software change. This means that the models are easier to maintain, evolve and understand.

\subsubsection{What, how and why?}

Since the advent of model driven software engineering it has become more necessary to develop techniques and tools to overcome this challenge of raising the level of abstraction in software evolution. Model driven architecture (MDA) allows us to separate platform independent model (PIM) from platform specific model (PSM). To take full advantage of MDA, we need more work and research on model evolution. Executable UML takes the concept of MDA further. It removes the PSM from MDA and 
directly generates code that can execute. To support executable UML, which is gaining popularity, we need to fully introduce evolution at the model level. Furthermore we must be able to answer the following questions. How can evolution affect and help code generation in executable UML? How can we implement a framework that can detect, trace and manage evolution in models and how can we support tools for managing evolution at this level? How can we automate model evolution? It is possible by introducing a model manipulation language. But what else do we need in that language for model evolution? Do we need to extend UML or use the existing UML specifications? It is easier to collect the necessary information, such as, the number of classes used, their operations and attributes etc, at the design level than at the source code level. How do we address change, including correction, improvement and enhancement at this level of abstraction?

\subsection{Dependency relationships between UML Class Model elements}

In UML, dependency relationships can be divided into four different relationships. These dependency relationships are described in Table 2.2. Many standard stereotypes for UML 1.x are now obsolete in UML 2.0. Table 2.1 lists all the stereotypes defined in [5] for dependency relationships in UML 2.0.

\subsection{Formal languages}

A language contains a collection of words or collection of sentences that can be formally defined by mathematical formulas and can be processed by a machine. If $F$ is a finite length of sequences of elements drawn from a finite set $S$ of symbols, then we can define a formal language $L$ mathematically as follows:

$$
L=\{S, F\}
$$


if $S=\{a, b\}$

then $F$ can be abbabbabb or abbabb

Table 2.1 Stereotypes for dependency in UML 2.0

\begin{tabular}{|c|c|c|}
\hline Name & Metaclass & Description \\
\hline$<<$ call $>>$ & Usage & $\begin{array}{l}\text { A call dependency specifies that an operation in the } \\
\text { source class invokes an operation in the target class. }\end{array}$ \\
\hline$<<$ create $>>$ & Usage & $\begin{array}{l}\text { A usage dependency denoting that the client classifier } \\
\text { creates instances of the supplier classifier. }\end{array}$ \\
\hline 《<rive $>>$ & Abstraction & $\begin{array}{l}\text { A derived dependency specifies that the client may be } \\
\text { computed from the supplier. }\end{array}$ \\
\hline $\begin{array}{l}<<\text { import>> } \\
<<\text { access }>>\end{array}$ & Permission & $\begin{array}{l}\text { A package import is a relationship that allows the use of } \\
\text { unqualified names to refer to package members from } \\
\text { other namespaces. <<import>> is public and <<access }>> \\
\text { is private. }\end{array}$ \\
\hline <<instantiate >> & Usage & $\begin{array}{l}\text { A usage dependency among classifiers indicating that } \\
\text { operations on the client create instances of the supplier. }\end{array}$ \\
\hline$<<$ merge $>>$ & Relationship & $\begin{array}{l}\text { A package merge defines how the contents of one } \\
\text { package are extended by the contents of another package. } \\
\text { It also defines package merge rules if certain elements in } \\
\text { both packages represent the same entity. }\end{array}$ \\
\hline <<refine >> & Abstraction & $\begin{array}{l}\text { Specifies a refinement relationship between model } \\
\text { elements at different semantic levels, such as analysis and } \\
\text { design. }\end{array}$ \\
\hline$<<$ send $>>$ & Usage & $\begin{array}{l}\text { A usage dependency whose source is an operation and } \\
\text { whose target is a signal, specifying that the source sends } \\
\text { the target signal. }\end{array}$ \\
\hline <<substitute〉> & Substitution & $\begin{array}{l}\text { A substitution is a relationship between two classifiers } \\
\text { which signifies that one Classifier complies with the } \\
\text { contracts specified by the other classifier. }\end{array}$ \\
\hline$<<$ trace $>>$ & Abstraction & $\begin{array}{l}\text { Specifies a trace relationship between model elements. } \\
\text { Traces are mainly used for tracking requirements and } \\
\text { changes across models. }\end{array}$ \\
\hline$<<$ use $>>$ & Usage & $\begin{array}{l}\text { A usage dependency in which one element requires } \\
\text { another element (or set of elements) for its full } \\
\text { implementation or operation. }\end{array}$ \\
\hline
\end{tabular}


Table 2.2 Dependency relationships between UML Class Model elements [5]

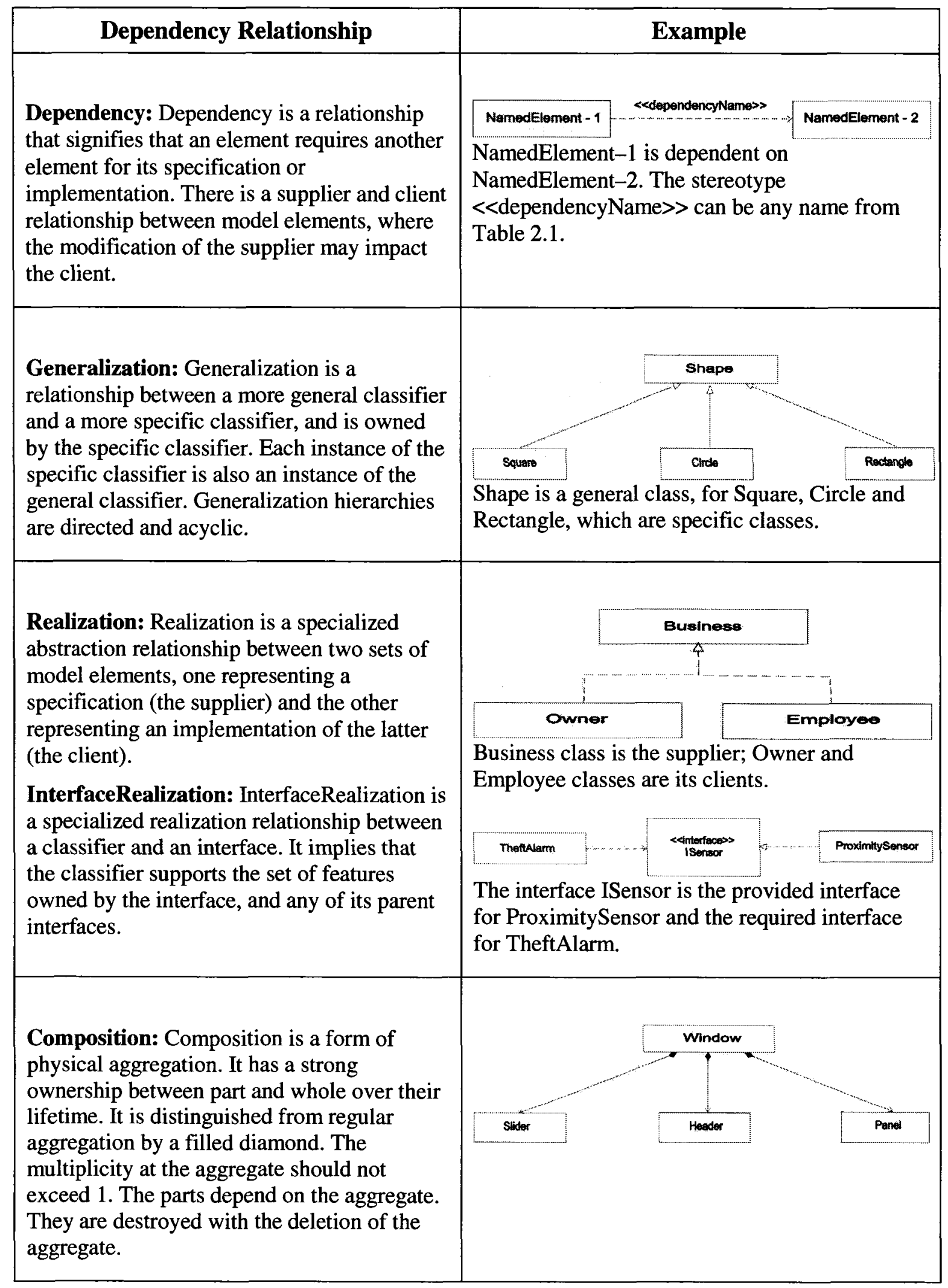




\subsubsection{Specification of formal languages}

As defined above $L$ is a set of strings over a set of symbols. It is desirable to consider languages that contain an arbitrary number of strings. We do not want to put an upper bound on the number of strings, so these languages cannot be specified by enumerating the set of strings in the language. Hence, we want our specification of the language to be finite although the language being specified is not finite. There are several techniques for language specification that fulfill this requirement. One of the techniques is to use a generative system known as grammar. A Grammar is a mathematical system for defining a language and a method for giving sentences in the language a useful structure. A grammar consists of a set of terminals, a set of non-terminals, a set of production rules and a starting symbol. Production rules are the heart of a grammar and are used to generate the sentences of the language. There are many grammar forms but most of the formal languages can be defined by a context free grammar (CFG) [6], and we have used CFG to define CAL. Another technique is to use a procedure that when given an arbitrary input string will halt and answer yes if the given input string is in the language and vice versa. This procedure will take a finite amount of time to compute the answer and is called a recognizer for the language. We will now look into the first technique and briefly describe the two most popular grammars used for specifying formal languages.

LL(k) grammar. This is the grammar for which left parsers (also called top-down parsers) [3] can be built deterministically if the parser is allowed to look $k$ input symbols (tokens) to the right of its current input position. Poorly designed languages usually have a very high $k$ value. If $k$ is one, then that grammar is called 
$L L(1)$ grammar. These grammars are very restrictive but very popular because the corresponding LL parsers only need to look at the next token to make their parsing decisions.

LR(k) grammar. This is the grammar for which right parsers (also called bottom-up parsers) [3] can be built deterministically if the parser is allowed to look $k$ input symbol (tokens) beyond its current input position.

Now we briefly describe how an $L L(k)$ grammar can be transformed into an $L L(1)$ grammar for efficient top-down parsing. A detailed discussion of this grammar transformation can be found in $[3,6]$.

\subsubsection{Left recursion removal in $L L(k)$ grammar}

A top-down parser for a grammar can loop forever if there is a production rule that has $A$ as a non-terminal of the form $A::=A \alpha$ for some string $\alpha$. This is known as left recursion. There are two types of left recursion as defined below:

(a) Immediate left recursion

$$
A::=A x
$$

(b) Indirect left recursion

$$
\begin{aligned}
& A::=B x \\
& B::=A y
\end{aligned}
$$

We can remove these left recursions from the production rule by using substitution, as explained in the dragon book [3].

\subsubsection{Left factoring}

Left factoring is a grammar transformation technique that is suitable for 
producing $L L(1)$ grammar. For example, in a production rule sometimes it becomes difficult to decide which alternative production to use to expand a non-terminal. To remove this ambiguity from the grammar, we rewrite the production rule such that we delay the decision until we have enough information. We explain this ambiguity with the following example:

$$
A::=z X \mid z Y
$$

In the above production rule we do not know whether to expand the non-terminal $A$ to $z X$ or $z Y$. The above production rule can be replaced by the following production rules to remove this ambiguity:

$$
\begin{aligned}
& A: \because=z A^{\prime} \\
& A^{\prime}: \because=X \mid Y
\end{aligned}
$$

\subsection{Executable UML}

Executable UML is a profile of UML that allows a developer to define the behavior of a single subject matter (a system) in sufficient detail, so that it can be executed [16]. These models are then woven together by an executable UML model compiler. An RFP (request for proposal) [9] is "Work in Progress" in OMG for defining executable UML. The objective of this RFP is to enable a chain of tools that supports construction, verification, translation and execution of computationally complete models. This proposal is for the definition of a computationally complete and compact subset of UML 2.0, to be known as "Executable UML Foundation," along with a full specification of the execution semantics of this subset. This compact subset is the selected metamodel of UML, which is as small as possible, that practically achieve computational 
completeness.

Executable UML comprises the class model, the state machines for the classes and the state procedures. These three models are expressed as UML class diagram, UML state diagram and action language. In late 2001, semantics for actions [27] were added to the UML, which provides a complete set of actions as a high level of abstraction. These defined semantics makes these three models computationally complete. Therefore a UML model specified using the above three models can be executed, verified and translated into implementation. This executable UML model is separate from any implementation and can be called platform independent model (PIM).

Model compilers are used to translate executable models into implementation using a set of decisions about the target hardware and software environment. A system can be divided into domains or subsystems. A domain can be added and can be modelled using executable UML. Each domain is independent of other domains in the system and hence can be replaced by another domain. Models of each domain are woven together by specifying a set of join points between the models. The two domains are joined together through the bridges. A bridge is defined as a layering dependency between domains. A domain makes assumptions and other domains take those assumptions as requirements. The domain keeps its autonomy using the bridges. Each domain is an aspect and each bridge is a set of join points, and these domains need to be woven together and translated to form the implementation by the model compiler. This is the same concept used by aspect-oriented programming (AOP) [51], at a different level of abstraction.

\subsection{OCL (Object Constraint Language)}

One of the shortcomings of UML is that it cannot be used for providing all the 
relevant information about the specification of a system. This relevant information is usually provided in a natural language, which is not very precise and hence is ambiguous. To remove this ambiguity we use formal languages. Because of the mathematical complexities of formal languages, it is very difficult for a non-programmer to use them for system specification. To make UML models more precise and comprehensive, OCL has been defined by the object management group (OMG).

OCL is not a programming language but a modeling language. OCL is a pure specification language: an expression in OCL simply returns a value and cannot change anything in the model. OCL is a typed language, and its expressions are written in the context of an instance of a specific type. OCL can be used as a query language. It can also be used to specify invariants and types, to describe pre and post conditions and to specify constraints on operations. The general form of an OCL expression is shown below:

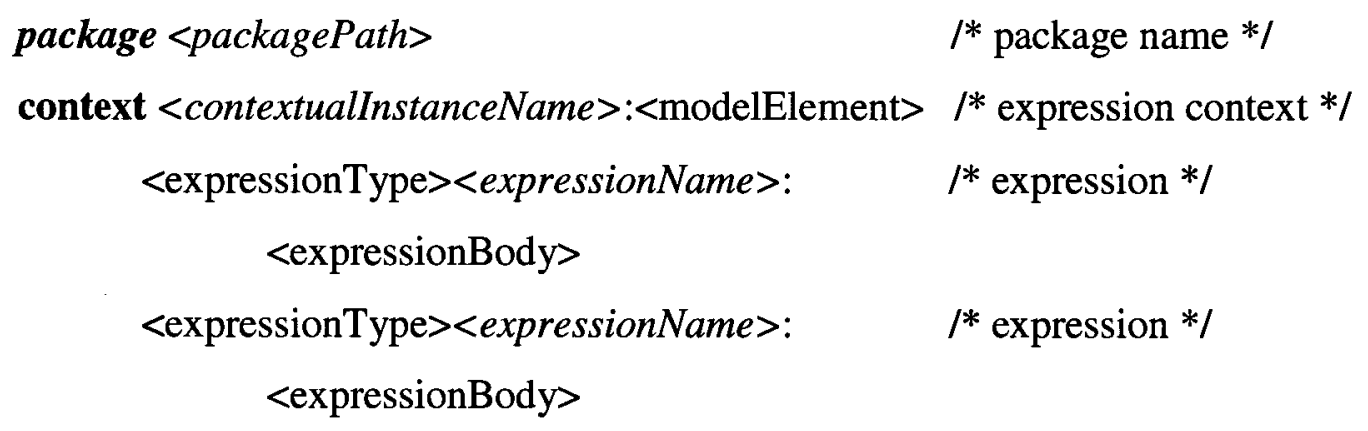

We use bold to show an OCL keyword and italic to show an optional element. 


\subsubsection{OCL collections}

Collection is a predefined type in OCL. There are four different Collection types in OCL, Set, OrderedSet, Sequences and Bag. A Set is a mathematical set. It does not contain duplicate elements and the elements are not ordered. An OrderedSet is a Set whose elements are ordered. A Bag is like a Set that may contain duplicate unordered elements. A Sequence is like a Bag in which the elements are ordered. These collections have an extensive set of operations for conversion, comparison, query, access, selection and iteration.

\subsubsection{OCL navigation}

Navigation is a process of following a link from a source object to one or more target objects. Starting from a specified object, we can navigate an association to refer to other objects and their properties. The properties defined in the OCL specifications [2] are an attribute, classifiers, association ends and a side-effect-free (query) method or operation. By default, navigation always results in a Set when multiplicity is greater than 1. Navigation across multiple associations always results in a Bag when multiplicity is greater than 1.

\subsubsection{OCL standard library}

The OCL standard library consists of built-in types, predefined types and operations. The built-in types are OclAny, OclVoid, OclInvalid and OclMessage. The predefined types include primitive types, Integer, Real, String, Boolean, and Collection types (also called OCL collections). OclAny is a super type for all the types except the OCL predefined Collection types. All these types have an extensive set of operations specified in [2]. This standard library is a mandatory part of OCL and any 
implementation of OCL must include this library package.

\subsection{TLA (Temporal Logic of Actions)}

TLA is a high-level specification language based on set theory and first order logic. This section gives an overview of TLA with the help of an example. More details on TLA can be found in [50]. A system is formally specified using a mathematical model. TLA is a tool for specifying the mathematical model of a system, which may have some behavioral properties. These properties can be functional and logical, and represent a correct execution of the system. A system can be described as a sequence of states. A state assigns values to the variables. While specifying a system's behavior we need to define an initial value, the variable's values as a relation of time and some constants.

TLA specification helps the design process and problems can be corrected in the design phase rather than after implementation. These specifications can be communicated in a clear and concise way and provide a valuable guide to engineers who implement and test the system. Since TLA specification is a formal description of the system, tools can be applied to find errors and test the system. TLC, a TLA model checker [50], is a tool that is written for this purpose and is used in this thesis to verify the CAL model specifications (see Chapter 5).

To see how TLA is used, we first start with a description of an example of a trivial system using TLA. This example is taken from the book [50] Specifying systems: the TLA+ language and tools for hardware and software engineers by Leslie Lamport. The example is a simple hour clock whose display cycles through the values 1 through 12. We can say there are 12 states in this system. If we represent the clock hour with $h r$, then the behavior of the clock can be defined as follows: 
$[h r=11] \rightarrow[h r=12] \rightarrow[h r=1] \rightarrow[h r=2] \rightarrow \ldots$

As explained above we need to specify initial values and a relation for next state formula. We can define predicates $H C$ ini and $H C n x t$, where the symbol $\triangleq$ means is defined equal to:

$H c i n i \triangleq h r \in\{1, \ldots, 12\}$

$H C n x t \triangleq h r^{\prime}=I F h r \neq 12 T H E N h r+1$ ELSE 1

While defining the formula for HCnxt we have used primed $\left(h r^{\prime}=\right.$ value in next state) and unprimed ( $h r=$ value in current state) variables. Such a formula is called an action. Action can be TRUE or FALSE after a state. When an action occurs, we say that action is executed. In this case, when HCnxt occurs we say that HCnxt is executed. Now we specify a single formula that asserts that the initial state satisfies $H C i n i$, and every other state satisfies HCnxt. For this purpose, TLA uses a temporal logic operator $\square$ (pronounced box). The temporal formula $\square F$ asserts that formula $F$ is always TRUE. So we can write a single temporal formula as

$H C \triangleq H C i n i \wedge \square H C n x t$

which is only true if the initial state satisfies $H C i n i$, and every other state satisfies $H C n x t$. This formula satisfies all the behaviors defined in Equation 2.1. There may be a state where $h r$ value does not change i.e. $h r^{\prime}=h r$. These are called stuttering steps of the clock and satisfy $H C n x t \vee\left(h r^{\prime}=h r\right)$. In TLA, this can be written as $\square[H C n x t]_{h r}$, and we can write the temporal formula in compact form as

$H C \triangleq H C i n i \wedge \square[H C n x t]_{h r}$ 
This formula also allows the following behavior with stuttering states:

$[h r=10] \rightarrow[h r=11] \rightarrow[h r=11] \rightarrow[h r=11] \rightarrow[h r=12] \rightarrow \ldots$

For the clock to behave properly, it should only display an integer from 1 to 12 in every state of any behavior satisfying the clock specification $H C$. HCini asserts that $h r$ is an integer from 1 to 12 , and $\square H C i n i$ asserts that $H C i n i$ is always TRUE and should be true for any behavior satisfying $H C$. Therefore, we can say the temporal formula $\square H C i n i$ implies $H C$ should be satisfied by every behavior. A temporal formula satisfied by every behavior is called a theorem, so $\square \mathrm{HCini} \Rightarrow H C$ is a theorem.

A typical TLA program consists of a module name, an extends statement, a declaration statement, operator definitions and a theorem. Figure 2.1 shows a listing of the example both in typesets and an ASCII version with the configuration file (HourClock.cfg). The version with typesets is line numbered from 1 through 6 . At the top, MODULE HourClock defines a module name, HourClock. The line 1 extends the Naturals module (library of natural numbers), which is one of the built-in modules of TLA. Any built-in or user-defined module can be extended and reused. Line 2 declares a variable. Lines 3, 4 and 5 are operator definitions. The last line defines a theorem. The ASCII version of the specifications can be tested and verified by TLC. In the next Section, we describe how and why an algorithm language based on TLA is used to describe a system that can be model checked by TLC.

\subsubsection{An algorithm language (pluscal) based on TLA}

The small set of CAL constructs hides the complexity of its internal model, but the tool that implements CAL needs to know this complex model. Therefore, we need to 
formally describe this model for the tools and also for the purpose of verification. The CAL data structure (ADAG), operations and actions can be directly specified in TLA but may need significant amount of space and time. To speed up this process and save space, in our research we found an algorithm language that is based on TLA and surprisingly named C-type Algorithmic Language (+CAL). Because of the similarity of its name with the language defined in this thesis, we call this algorithm language as pluscal. In this section we briefly give an overview of pluscal.
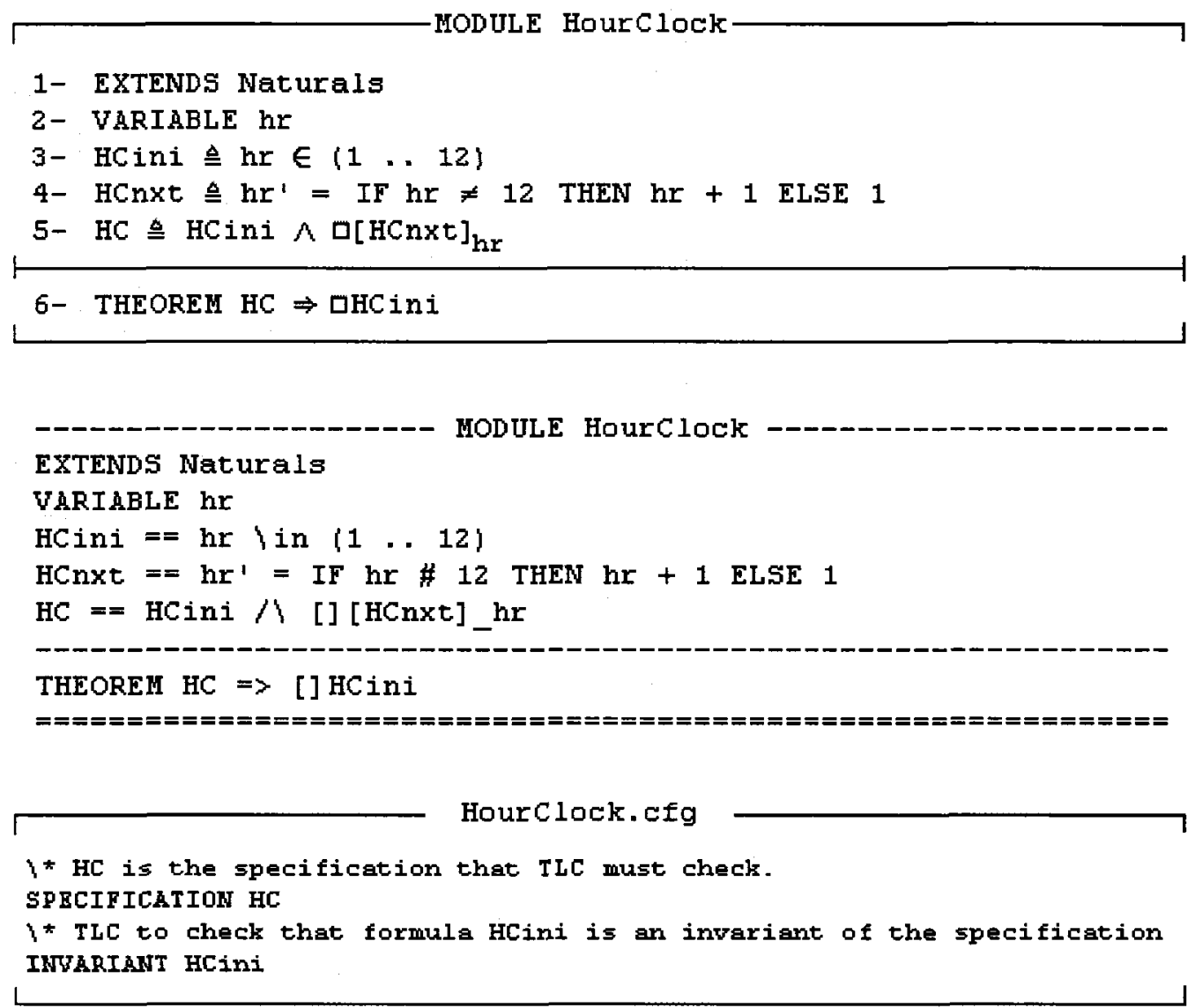

Figure 2.1 Simple clock example from [50]

As described above pluscal is based on TLA. There are two alternate syntaxes for 
pluscal. One is p-syntax and the other is c-syntax. Since $c$-syntax is new, more compact, and closely resembles C\# and Java, we have used c-syntax in this thesis to describe CAL specifications. TLA expressions can be combined with pluscal expressions.

Figure 2.2 (a) shows a Euclid algorithm specified in pluscal using c-syntax. It computes the greatest common divisor (GCD) of two numbers. A procedure has been used to implement the algorithm, which is called in the main block. The main block is enclosed in brackets. If a procedure is used then we need to extend Sequences, to be used during translation for maintaining stack and other variables, as Sequences for the procedure. $T L C$ is a built-in module for basic operations like print etc. The procedure has two labels, RunEuclid1 and RunEuclid2. The TLC translates these labels into operator definitions in TLA. Each procedure should have a return statement at the end. In the define block, the user can define TLA expressions like TypeInvariant and other userdefined operators.

The configuration file is shown in Figure 2.2 (b). It sets the value of $\mathrm{N}$ to 9 and declares the INVARIANT, as TypeInvariant, and the SPECIFICATION, as Spec, to be checked by the TLC. Although Spec defined in the Euclid.tla file cannot be seen, it is defined automatically by the TLC translator. Every state (operator) and call to a procedure in the main block is a Spec. For example, in Figure $2.1 \mathrm{HC}$ at line number 6 is a Spec. Figure 2.2 (c) is the output when Euclid.tla is compiled and executed by the TLC. The TLC generates 9 distinct states, and the value of $v$ in file Euclid.tla changes from 1 to 9. That is to say, the $G C D$ procedure is executed 9 times, and we see 9 different print outputs. 
MODULE Eudid

EXTENDS Naturals, Sequences, TLC

CONSTANT $N$

(*)

-algorithm Euclidalgorithm

variables $\mathrm{u}=24 ; \mathrm{v}$ in $1 . . \mathrm{N}$

define $1 *$ Define block \}

Typelnvariant $==\wedge u$ in $\operatorname{Nat} \wedge * \operatorname{lin} 1 \ldots N$

(*efinition of procedure

procedure GCD $(n 1, n 2)$

variables $v$ ini $=n 2$ i

t

RunEudid: while (n1 0 )

t

if $(n 1<n 2)$

t j. $n 1:=n 2|| n 2:=n 1$ t* swap $n 1$ and $n 2$ RunEuclid2; $n 1: m n 1=n 2$

print 4 "GCD of ", 24, *_ini, "is", n2>>; return;

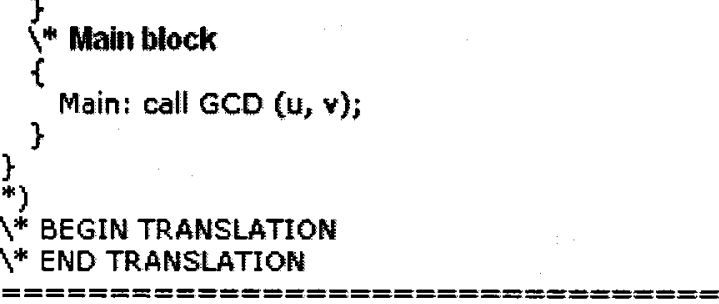

a. Fuclid.tla
CONSTANT $\mathbf{N}=9$

INVARIANT TypeInyariant

SPECIFICATION SpEC

b. Enchidefg
Finished computing initial states: 9 distinct states generated.

\& "GCD of ", 24, $8, " i s^{*}, 10>>$

<e "GCD of ", 24, 6, "is", 6>>

$<4$ "GCD of " $24,9, "$ is $", 3>>$

<4 "GCD of " $24,4, "$ "is", $4>>$

ex "GCD of ", 24, 3, "is*, $3>3$

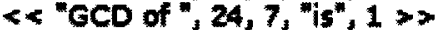

ee "GCD of ", 24, 5, "is", $1>>$

$<4$ "GCD of ", 24, $2, "$ is", $2>>$

$\ll{ }^{*} 6 \mathrm{CD}$ of ", $24,1, \mathrm{~m}^{*} \mathrm{~s}^{*}, 1$, $>$

Model checking completed, No error has been found

c. TLC Output

Figure 2.2 Euclid algorithm in pluscal

TLC is implemented in Java and requires a Java Runtime Environment (JRE) to run. The version of JRE depends on the current implementation of the TLC. The following commands were used to run the TLC translator and model checker for the file Euclid.tla with JRE version 1.5:

java pcal.trans euclid.tla

java tlc.TLC -cleanup -difftrace euclid.tla 


\subsection{Related work in model evolution}

Most software evolution techniques target the source code level. There is much less support at a higher level (models) of abstraction. The major reason for this is the lack of support tools for model evolution. In this Section we discuss current efforts at automating model evolution, starting from evolution contracts by Tom Men and Theo D'Hondt in the year 2000, to the most recent efforts. A comparative analysis is done with the approach and techniques used in this thesis. The two most recent efforts, C-SAW from Vanderbilt University and the iACMTool by L.C. Briand and Y. Labiche, are compared in Section 2.7.3. Since CAL is an extension of OCL 2.0, other OCL tools have been listed and compared on the basis of the platform they support.

\subsubsection{Evolution contracts}

One of the earlier efforts to automate software evolution in UML was done in 2000 [28]. Tom Mens and Theo D'Hondt proposed to extend the metamodel of UML 1.3 and based on this extension defined evolution contracts using stereotypes such as $<<$ add $>>,<<$ remove $>>,<<$ connect $>>$ and $<<$ disconnect $>>$ etc. The purpose of these contracts is to automatically detect conflicts. The main idea behind this is to define a formal contract between the provider and the modifier for the evolution. They mentioned about creating a conflict table, which describes what kind of conflicts occur in different cases of conflicts, to automate the conflict detection. There is no specific detail about the table or the kinds of conflicts. Neither the data structure for storing such a table nor how the data structure was created is mentioned in the paper. The authors claim that they implemented an evolution contract framework in PROLOG for checking evolution conflicts, but no case study or example, or reference to a case study or an example, is 
given in the paper.

\subsubsection{Refactoring}

Recently there have been many efforts at implementing refactoring [13] at the model level, which are described below. Only the efforts relevant to the thesis are included here.

The applicability of Action Semantics (AS) [27] to UML 1.4 metamodel is reviewed in [12], and an approach is presented for using AS for meta-programming to perform model transformation. The authors used this approach to propose the implementation of refactoring in UML. Their approach combined AS with OCL to verify whether a transformation may be applied to a given context. It also illustrated the use of AS for the implementation of refactoring. They show how this approach can be used to implement refactoring, which emphasizes the importance of the use of OCL with AS. The method used in this approach divided the model transformation into two types, model manipulation and code generation. Model transformation has two parts, the selection of model elements concerned and the actions performing the transformation itself. Since there is no formal specification given in the paper for the actions, we cannot analyze this approach further. Also the approach is based on meta-programming, and is not easily used by a non-programmer.

The authors in [14] applied the concept of refactoring at a higher level of abstraction. They developed a model refactoring tool where users can specify the transformation rules. This tool is front-end of a transformation engine, constraint specification aspect weaver (C-SAW), which is a plugin within Generic Modeling Environment (GME). The tool, C-SAW and GME [20, 21] are discussed below and 
compared in Section 2.7.5.

Refactoring in executable UML is discussed in [15]. The computer-aided software engineering (CASE) tool used is TAU from Telelogic. A specification template for refactoring is proposed. OCL is used to formally define the specification for refactoring. The changes are carried out based on pre and post conditions. If pre conditions are met, then the transformation is carried out. The transformation is verified using the post conditions. To make these changes a TAU model is manipulated using component object model (COM) application programming interfaces (API's) implemented in Borland Delphi's Object Pascal. Although the approach used is very practical, the methodology used and the implementation are based on Telelogic TAU, and so it is not interoperable. For a non-programmer the use of COM API's is not easy.

Because the previous efforts we have discussed were concerned with refactoring, no impact analysis was carried out before making changes in these studies. In what follows, we describe two of the recent research efforts in model evolution for impact analysis. Then we discuss two other recent research efforts on modeling languages for model transformation and see if they provide any support for model evolution in the language.

\subsubsection{Model evolution and impact analysis}

L.C. Briand, Y. Labiche and L. O'Sullivan $[22,23,31]$ proposed an approach to perform impact analysis of UML model elements and present promising empirical results. This is the first empirical effort at automating impact analysis of UML models, but it does not address making changes to the model. The changes in two versions of the model are automatically identified and reported. The impacted elements, whether direct 
or indirect, are determined using impact analysis rules defined in OCL. The distance is measured between the changed element and the impacted element, to prioritize the impact analysis strategy. If the distance is large, then the chance of impact is less likely. The distance measured between the changed element and the impacted element is defined as the number of impact rules applied to identify the impacted element. These rules are used to trace the "impact of a change" as defined in definitions 2 and 3 in [22], so we can say this approach uses a traceability analysis technique for impact analysis. A prototype tool, iACMTool, has been implemented to automate the process. Impacted model elements are detected in the form of OCL collections (as Bags) by using 97 rules [31] defined in OCL. Since an element may be impacted by several others, these OCL collections may contain several occurrences of the impacted element, which greatly affects the efficiency of the tool implemented.

In the research paper [17], impact analysis is performed using traceability analysis, which unlike dependency analysis gives a coarse evaluation of relationships between program entities. However, the authors claim that their traceability analysis technique gives detailed guidance as to what traces should be established to support impact analysis. Their trace model is based on three relationships: representation, refinement and dependency. They use the Rhapsody CASE tool, from Telelogic, and a requirement management tool, RequisitePro (also a commercial tool). Both these tools are linked using the COM API's of each tool. They call this tool environment QuaTrace, which supports requirements engineers, project planners and software maintainers. The tool is good for tracing changes in requirements and documents, neither of which needs a detailed analysis of dependencies. QuaTrace is highly dependent on other tools and is not 
interoperable, and the use of API's makes this approach difficult to implement for a nonprogrammer.

\subsubsection{Recent languages for model transformation}

Naoyasu Ubayashi and others developed the aspect-oriented modeling language AspectM (aspect for modeling) [18]. AspectM is used [19] to demonstrate the effectiveness of aspect-orientation in terms of model evolution. That is, the model can be evolved by adding aspects as concerns, like security etc. AOP $[30,51]$ is based on Join Point Model (JPM), which includes join points, pointcuts and advice. The authors used the same concept at the modeling level. Advices can be, add class, delete class, add operation, delete operation, add attribute and delete attribute etc. Since these aspects or concerns cross-cut many places in the model, it is very important to perform impact analysis, before adding or removing these aspects. AspectM is a model compiler that supports modeling level aspects and is defined as an Extendible Markup Language (XML) based AOP language. JPM can be described in XML or AspectM notations (diagrams). AspectM supports the MDA process and weaves UML and aspect diagrams together into PIM using aspect libraries to generate PSM. However, AspectM only supports adding aspects that are not dependent on others and performs no impact analysis. It does not support pre and post conditions or invariants, and hence there is no support for defining contracts. The authors are working on extending AspectM to add support for defining contracts. They proposed a language, contract writing language (COW) [29] that can be used for defining contracts for weaving aspects. It is implemented for AspectJ [30]. Using these contracts, a programmer can specify how a program should behave before and after weaving. The work is in progress for applying 
these contracts for impact analysis. AspectM is more of a model transformation language using aspects. Since AspectM does not support model evolution analysis, and the work is in progress, we do not discuss it here any further.

C-SAW [20] from the Institute for Software Integrated Systems (ISIS), Vanderbilt University, is a transformation engine for manipulating models that shows how tools can be used to automate model evolution. A tool (an engine) is developed [14] to specify the transformation rules to act as a front end to C-SAW. This engine is called an aspect weaver. C-SAW is a plug-in for Vanderbilt University's GME, a configurable toolset that supports domain specific modeling environments. The aspects and strategies for model weaving are based on the language ECL (embedded constraint language) [21]. It is used for describing the location and behavior of the change to be performed on the source models. Like CAL, it provides operators to change the model, addModel, addConnection, removeModel and removeConnection etc. ECL is more of an aspect oriented language like AspectM [18], for model transformation. There are two types of constructs in ECL, a strategy for defining a procedure for transformation and an aspect for defining an entry point of model transformation. Transformation rules (behavior of the change) can be specified in the form of a strategy, but it is not clear if any impact analysis is performed before transforming or making changes to the model, which is very important for maintaining software. This approach is very useful in applying multiple changes to the model at one time. ECL is implemented using GME and uses the same type system (a graph model), so unlike $\mathrm{CAL}$, it is a domain specific language. A parser is implemented to generate an abstract syntax tree (AST), and an interpreter is implemented to traverse the AST and to perform transformation using modeling API's provided by 
GME. ECL borrows some concepts from OCL but is not a complete extension of OCL. There are no OCL-like syntaxes for pre and post conditions for defining contracts in ECL. Only pre conditions can be applied inside strategies using a different syntax than OCL.

\subsubsection{Comparison}

In this Section, we compare the research that is most recent, as described in the above sections, with the approach and techniques used and the tool implemented in this thesis. Following is a comparison with the approach proposed by L.C. Briand and Y. Labiche [22]:

- The major difference is that iACMTool uses a traceability analysis technique, and CAL uses a dependency analysis technique for impact analysis.

- The approach described needs to compare two complete models to detect changes. That is, the original model and the changed model are required to complete the detection process. We use agile methods [10]. That is, a change or few changes are made and then analyzed, to keep up with the ongoing changes to the requirements. Our approach works on just one model. As the model is changing the tool is analyzing the change. It makes the analysis process practical, dynamic and compact.

- Both the approaches are formally defined and can be implemented independently or integrated into any UML tool, as is shown by their prototype tool implementations.

- The use of constraints, as rules, defined in OCL by itself is not optimal for 
impact analysis. Invoking constraints each time for tracing the change of a UML model element is not efficient. For example, in [22] select and equals have been used to query the model and to match the pattern respectively. These rules generate a list of Bags and a counter whose number prioritizes impact analysis. The tool also generates an impact analysis report. Since CAL is a complete extension of OCL, the same rules can be used and written in CAL. In addition CAL defines an efficient data structure, ADAG that can be viewed as a dependency analysis tree. This view is helpful for software engineers and maintainers to get a full picture of the dependency tree and make early software design and maintenance decisions. It can also be interacted with to make changes.

- Distance has been used in [22] to prioritize impact analysis. The CAL data structure, ADAG, is also a transitive closure of relations, so the distance between any two model elements in the ADAG gives the same distance. In addition, we are assigning weights to each element in the ADAG so that each dependency relation can be counted according to its weight in the dependency path.

Following is a comparison of CAL with C-SAW and ECL $[20,21]$ from the ISIS, Vanderbilt University:

- Our research indicates that C-SAW and ECL perform model transformation without doing any impact analysis of the changes being made. The approach 
used is more like software automation for changes in a GME model than software evolution.

- $\quad$ C-SAW is a plug-in for GME, which is a domain-specific modeling toolset. It is based on the GME graph model and uses GME API's to manipulate models. That is, C-SAW and ECL are dependent on another toolset, GME, and cannot be integrated into other tools or used independently.

- $\quad \mathrm{ECL}$ is more of an aspect-oriented language like AspectM [18]. Using aspects and strategies, it can be used for making changes to the GME model. Although it is not mentioned in the paper, strategies can be used to search for dependent elements. The same can be achieved by using constraints in OCL. However, ECL has never been used for this purpose, and it is not possible to obtain all the direct and indirect dependencies using this approach.

- ECL automates the process of transformation using aspects and strategies as described above and needs an aspect weaver, such as C-SAW. If a change is made in one of the aspects then, how does that change affect the weaving with other aspects? How does that change affect the weaved model? Aspects are added assuming that they are independent of other aspects in the model, but can we add and delete aspects that are dependent on others? In reality software evolution is about changes made to the software that can either be dependent or not on other objects. There is no mention of such support in ECL or C-SAW while defining aspects or strategies. These questions may open up another research area for software evolution in aspect-oriented modeling (AOM) or in domain-specific languages (DSL). 
- ECL is not a full extension of OCL. The syntax of ECL is different than OCL. Only pre conditions can be applied inside strategies on GME models. There is no verification of the changes made using ECL. Verification is only done through GME. CAL is a complete extension of OCL, and changes can also be verified using OCL constraints.

\subsubsection{Impact analysis support in commercial UML tools}

This Section gives a brief description of commercial tools that can be used for impact analysis of UML models. Rhapsody [32] provides COM API's that developers can use for impact analysis. Rational in some of its products like Rational Rose [33] and XDE [34] provides API's that can be used in Java, Visual Basic or C\# to manipulate a UML model. These API's can also be used to develop a separate application or a tool, or can be integrated within the project to perform impact analysis. Other tools (see Section 3.5) have their own action languages, which can be used for model execution and transformation but not for impact analysis. Rhapsody Gateway [35] can be used for impact analysis. Gateway is only a requirements traceability tool that is a Rhapsody addon. It uses traceability techniques to perform impact analysis. There are significant disadvantages and practical concerns connected with these tools and the options they provide which are summarized below:

- It takes much effort and skill from an average software engineer who is not a programmer to develop an impact analysis application or a tool using the API's provided by the tool vendors. If not developed properly the application 
or the tool may need to be developed or changed for each project.

- Because of the limited capabilities of these API's the developer may not be able to define impact analysis rules at a higher level of abstraction.

- The tools and the options these tools provide are not automatic or portable and are highly dependent on the specific tool.

- There is a need to automate these tasks and make them flexible, interoperable and independent of any CASE tool.

Table 2.3 lists the tools that support OCL, including commercial, academic and open source, with a brief description of each tool. Only those tools are included that support OCL 2.0. The reason for listing them here is not to compare their technical features, but to compare the platform they support and where they are used (i.e. the license). Unlike the CAL parser, all the academic and open source tools are implemented in Java. Commercial tools are implemented using C++. This shows that the OCL tools that are being used in industry are still implemented in $\mathrm{C}++$. Almost all the commercial CASE tools that support UML are implemented in $\mathrm{C}++$ because of the graphical nature of the UML diagrams and notations. With the increasing speed of a central processing unit (CPU) and a graphics processing unit (GPU), this may change in the future. 
Table 2.3 Current OCL tools

\begin{tabular}{|c|c|c|c|c|}
\hline Tool Name & Description & Company & Platform & License \\
\hline Octopus & $\begin{array}{l}\text { An OCL plugin for } \\
\text { eclipse }\end{array}$ & $\begin{array}{l}\text { Klasse Objecten } \\
\text { octopus.sourceforge } \\
\text { net }\end{array}$ & Java & Open Source \\
\hline Oclarity & $\begin{array}{l}\text { Add-in for Rational } \\
\text { Rose to support OCL }\end{array}$ & $\begin{array}{l}\text { EmPowerTec } \\
\text { www.empowertec.d } \\
\text { e/products/rational- } \\
\text { rose-ocl.htm }\end{array}$ & $\begin{array}{l}\text { Rational } \\
\text { Rose } \\
(\mathrm{C}++)\end{array}$ & Commercial \\
\hline $\begin{array}{l}\text { Dresden OCL } \\
\text { Toolkit }\end{array}$ & OCL parser & $\begin{array}{l}\text { Dresden University } \\
\text { of Technology } \\
\text { dresden- } \\
\text { ocl.sourceforge.net }\end{array}$ & Java & Open Source \\
\hline $\begin{array}{l}\text { OSLO (Open } \\
\text { Source Library } \\
\text { for OCL) }\end{array}$ & $\begin{array}{l}\text { Based on the OCL } \\
\text { implementation of the } \\
\text { University of Kent }\end{array}$ & $\begin{array}{l}\text { University of Kent } \\
\text { oslo- } \\
\text { project.berlios.de }\end{array}$ & Java & Open Source \\
\hline Bold for Delphi & $\begin{array}{l}\text { Add-in to Borland } \\
\text { Delphi IDE to support } \\
\text { OCL }\end{array}$ & $\begin{array}{l}\text { Borland Software } \\
\text { Corporation } \\
\text { info.borland.com/te } \\
\text { chpubs/delphi/boldf } \\
\text { ordelphi }\end{array}$ & $\begin{array}{l}\text { Delphi } \\
(\mathrm{C}++)\end{array}$ & Commercial \\
\hline The KeY Project & $\begin{array}{l}\text { It is a formal software } \\
\text { specification and } \\
\text { verification tool. OCL } \\
\text { is used as a basis for } \\
\text { reasoning about the } \\
\text { specification. It requires } \\
\text { Borland Together for } \\
\text { UML / OCL support. }\end{array}$ & $\begin{array}{l}\text { University of } \\
\text { Karlsruhe } \\
\text { i12www.ira.uka.de/ } \\
\text { key }\end{array}$ & Java & Academic \\
\hline $\begin{array}{l}\text { OCLE (Object } \\
\text { Constraint } \\
\text { Language } \\
\text { Environment) }\end{array}$ & OCL tool & $\begin{array}{l}\text { University of City- } \\
\text { Napoca } \\
\text { lci.cs.ubbcluj.ro/ocl } \\
\text { e/index.htm }\end{array}$ & Java & Academic \\
\hline $\begin{array}{l}\text { OCL Compiler } \\
\text { Version } 1.5\end{array}$ & $\begin{array}{l}\text { This is a demo version } \\
\text { and an industrial } \\
\text { version is under } \\
\text { development }\end{array}$ & $\begin{array}{l}\text { Cybernetic } \\
\text { Intelligence } \mathrm{GmbH} \\
\text { www.cybernetic.org }\end{array}$ & Windows & Commercial \\
\hline
\end{tabular}




\section{Chapter 3}

\section{CAL}

Constraints with action language (CAL) is a text based, scripting language defined for the purpose of model evolution, and can be extended and used as an action language or in model compilers for incremental recompilation and retransformation of models, in model driven architecture (MDA) $[11,47]$. CAL contains a small set of constructs, but is powerful enough to be used efficiently for typical software evolution management operations like impact analysis, correction, improvement and enhancement of models. CAL is an extension of object constraint language (OCL) and is not specifically defined for UML. It supports generating the Annotated Directed Acyclic Graph (ADAG) of the model for dependency analysis.

The current implementation of the prototype tool VCAL (visual CAL), which will be discussed in Chapter 6, interprets CAL statements and then uses an Extensible Markup Language Metadata Interchange (XMI) representation of Unified Modeling Language (UML) to manipulate UML Class Models. The CAL parser also to be discussed in chapter 6 is implemented separately from the tool VCAL that uses it. In CAL we have extended OCL and added 5 actions, 10 operations and 1 data type (the data structure ADAG). Since CAL is a complete extension of OCL, we do not describe OCL types, operations, library or usage. Interested readers are referred to Chapter 2 and OMG document [2] for readings on OCL. 
In the present chapter, we discuss the extended CAL grammar, library and usage, and compare it with other action languages for software models. We also describe using OCL-like syntax CAL actions, operations and its data type. Within this description, the word result is used to refer to the value that is obtained from evaluating the operation and iterate is any searching technique (e.g. depth first search or breadth first search etc.) suitable for a directed acyclic graph (DAG). Post conditions are used to describe the properties of the result. At the end of this chapter, we compare CAL with other similar languages (action languages). Before describing CAL in detail, we first, list its basic characteristics:

- Complete extension of OCL, so constraints can be used with actions. Hence, CAL combines both declarative and imperative programming paradigms.

- Automation for dependency analysis.

- Easy to learn. Abstract the details so even a non-programmer in a business environment can use it effectively.

- Well defined grammar in a standard EBNF [1] form.

- Formal specifications in TLA [50] for better comprehension, implementation and verification.

- CAL parser implemented in $\mathrm{C}++$, which can easily be integrated into other modeling tools.

\subsection{CAL grammar}

There are commercial and open source tools that support OCL that are listed in Table 2.3. None of the open source parsers is in C++, and the OCL commercial tools are 
highly dependent on the tool vendor IDE. There is no open source OCL grammar for LL(1) parsing, so we decided to start from scratch and write our own grammar for OCL and extend it for CAL. We have used formal grammar to define the language for its obvious advantages of making parsing and translation of CAL simpler. The grammar for OCL 2.0 has been extracted from the OMG document [2]. The extracted grammar has been extended and production rules have been added for different actions. The CAL grammar can be defined by a 4-tuple $G=(T, N, P, S)$, where

$$
\begin{aligned}
& \mathrm{T}=\text { set of terminals; } \\
& \mathrm{N}=\text { set of non-terminals; } \\
& \mathrm{P}=\text { set of production rules; } \\
& \mathrm{S}=\text { an element of } \mathrm{N}, \text { a distinguished starting non-terminal. }
\end{aligned}
$$

The CAL grammar has been optimized for LL(1) parsing by removing left recursion and using left factoring in the parser, as explained in Section 2.3 and 6.2. In the process, a new set of production rules, such as LogicalStmt, RelationalStmt, AdditiveStmt, UnaryStmt and MultiplicativeStmt, which are not defined in the concrete syntax of OCL [2], has been added in the grammar for different operations.

The complete listing of the CAL grammar in EBNF [1] is given in Appendix A. There are three basic statements in the grammar, OclStmt, ActionStmt and DefStmt. A CAL file starts with package and ends with endpackage. In between comes the statements, which are of three types, attrOrAssocContext, classifierContext and operationContext. They all end with either DefStmt, OclStmt or ActionStmt.

\subsubsection{Production rules added for CAL}

There are five kinds of action statements that have been added for CAL: 
createStmt, deleteStmt, addAttrStmt, createLinkStmt and deleteLinkStmt. As each name suggests, they can be used to create or delete methods or attributes, and create or delete links or associations between elements. These production rules have been added to perform model evolution (change) tasks, and they are informally defined and explained below as CAL actions. The deleteStmt deletes both operation and attr (id can be the name of an operation or an attr). Figure 3.1 shows a complete list of the rules for these statements. Action statements can be used only in an operation context. We also list in

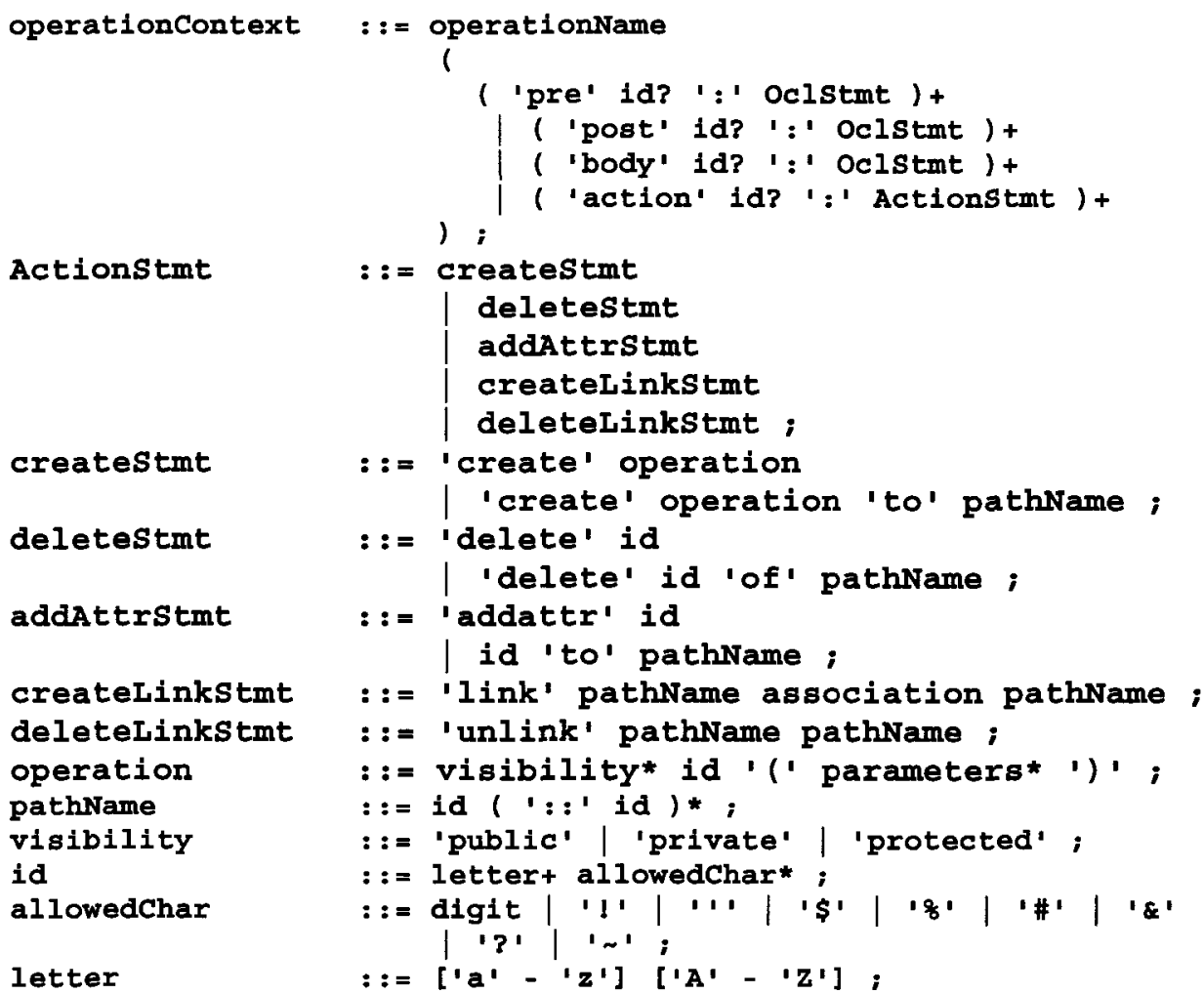

Figure 3.1 Production rules for CAL

Figure 3.1 the operationContext where ActionStmt has been defined. Pre conditions should come before and post conditions should come after the action statements. In one operation context there can be more than one action statement. An action statement is 
only valid for the operation context in which it is used and cannot be used outside that operation context.

\subsection{CAL library}

Like OCL, CAL is a typed language, so we have defined another collection type DagType for the CAL data structure ADAG. In addition to the OCL library defined in [2], CAL contains an additional library function Dag. This library function can be used to create the ADAG of a UML Class Model as defined below in Section 3.3.1. The methods depend and descendants, defined below, check for dependency between two elements and all the descendants of an element respectively. The production rules deleteStmt and deleteLinkStmt added for CAL check the dependency using the library function depend, which is defined in section 3.2 .9 , and accordingly the dependent objects are deleted or unlinked. The collection type DagType in CAL is used to define the ADAG. The complete process of using the ADAG can also be displayed visually to let the software design or maintenance engineer decide before making any changes to the model. In the next sections, we present the definitions of all the types and operations in the CAL library. These definitions use one of the ADAG operations contains, which is defined in Section 4.3.

\subsubsection{DagType}

DagType is a collection type, a place holder for the ADAG and a subclass of CollectionType. It can be used to create the ADAG using the following syntax. The name of DagType is Dag followed by the element's name:

inv: self.name $=$ 'Dag $f^{\prime}+$ self.element.name +$\}^{\prime}$ 


\subsubsection{Dag}

Dag is a DAG of an instance of the context (see Section 3.3.1 for the syntax and Section 3.4 for an example). The instance becomes the root vertex, and all its dependent vertices become the child vertices in the form of a DAG. DagType $D$ is represented by a tuple of four: $V$ the set of all the vertices, $E$ the set of all the edges, $L$ the set of all the labels and $W$ the set of all the weights. We call $D$ the annotated DAG (ADAG), which is the CAL data structure, and is formally defined as follows:

$D=(V, E, L, W)$

Where $E=(x, y)=\{x y: x \in V, y \in V, x y \in E\}$

such that

$E$ is a Partial order (transitive and non-reflexive) on set $V$

$L=\{(l(x) \cup l(y)) \in L: x y \in E\}$

$l(x)$ is the label of $x$ and $l(y)$ is the label of $y$. Read chapter 4 for an example and how to compute the label

$W=\{w(x, y) \in W: x y \in E\}$

$w(x, y)$ is the weight of $x$ that is based on the relation $(x-->y)$ between $x$ and $y$. The rule for computing $w(x)$ is formally defined in section 5.1 .

Total weight of $v \in V$ is defined as

$w(v)=\sum_{(a, b) \in D(v)} w(a, b)$

where $D(v) \in E$ and is a set of descendants of $v$ 


\subsection{3 isdag}

This operation returns TRUE if self is DagType; else it returns FALSE.

Context Dag::isdag() : Boolean

post: result $=$ self $->$ type $-->$ elementType $->$ oclIsKindOf(DagType $)=0$

\subsection{4 size}

This operation computes the number of vertices in the ADAG.

Context Dag::size() : Integer

post: result $=$ self-->iterate $($ elem; count $:$ Integer $=0 \mid$ count +1$)$

\subsection{5 isempty}

This operation returns TRUE if the ADAG is empty; else it returns FALSE.

Context Dag::isempty() : Boolean

post: result $=$ Dag $-->$ size ()$=0$

\subsection{6 flatten}

This operation returns the same element if the element type is not the DagType. If element type is a DagType then the result is an OrderedSet containing all the elements of self.

Context Dag::flatten() : OrderedSet(T) 
post: result $=$ if $($ self- $->$ isdag ()$)$

then

self-->iterate(elem; $s: s-->$ union(elem-->asOrderedSet()))

else

self

endif

\subsection{7 includes}

This operation checks if object $T$ is present in the ADAG.

Context Dag::includes (object : T) : Boolean

pre: self-->isdag() and (not self-->isempty())

post: result $=\operatorname{self}->\operatorname{contain}(T)=1$

\subsection{8 excludes}

This operation checks if object $T$ is not present in the ADAG.

Context Dag::excludes(object : T) : Boolean

pre: self-->isdag() and (not self-->isempty())

post: result $=$ self $->\operatorname{contains}(T)=0$

\subsection{9 depend}

This operation returns TRUE if an object parent is dependent on an object child. 
These two objects must be elements of the ADAG. It computes the mod of two elements to check for dependency. If mod is zero, then the two elements are reachable, i.e. dependent on each other (parent --> child). The post condition computes the mod of labels of the object parent and the object child. The ADAG is annotated (labeled) in such a way that if any two labels are divisible then the two objects are dependent and vice versa. Hence, if the mod is equal to zero, then the two objects are dependent. This improved labeling scheme for the ADAG is described in Chapter 4.

Context Dag::depend(object parent, object : child) : Boolean

pre: self-->isDagType() and self-->includes (parent)

$$
\text { and self-->includes (child) }
$$

post: result $=($ if label $($ parent $)>\operatorname{label}($ child $)$

then

label(parent) mod label(child)

else

label(child) mod label(parent)

$$
\text { endif ) }=0
$$

\subsubsection{0 descendants}

This operation returns all the descendants of the object $\mathrm{E}$ as an ordered set.

Context Dag::descendants(object E) : Boolean

pre: self-->isDagType() and self-->isempty() 
post: result = self-->iterate (elem; s:self-->depend $(E, s))-->$ flatten ()

\subsubsection{1 view}

This operation creates a view of the element $E$ as the root element and returns TRUE if a view is created. The view displays, either as text or graphics depending on the tool that implements the compiler, the ADAG with all its vertices and edges. A user can use this view to perform all $5 \mathrm{CAL}$ actions on this interactive ADAG.

Context Dag::view (object : E) : Boolean

pre: self-- >isdag() and $\sim$ self-->isempty()

post: result $=$ Dag $\{$ E $\}-->$ view( $)$

\subsection{CAL actions}

CAL actions, create, delete, link, unlink and addattr, are responsible for incrementing the global variable CHANGE_RATE (global variable defined in the CAL library). CHANGE_RATE is the number of objects changed and can be used to calculate the percentage of objects that changed from one version to the next, which is called change rate [4]. Similarly create and delete increment or decrement the global variables OBJECTS_ADDED and OBJECTS_REMOVED respectively to calculate the growth rate [4], which is the difference between added and removed objects. The increment and decrement of the global variables CHANGE_RATE, OBJECTS_ADDED and OBJECTS _REMOVED are not part of the definitions. They are part of the 5 DAG operations used in the definitions. One of the actions, create, and its sub-actions, setweight and relabel, 
are formally specified in TLA+ for verification in Chapter 5, so we do not define setweight and relabel in this section. In this section, we informally define all the CAL actions in OCL-like syntax. These definitions use CAL operations (isdag, size, includes, excludes, depend and descendants), which are defined above, and DAG operations (add, delete, setParent and removeLink), which are defined in Section 4.3.

\subsection{1 create}

This action adds an object to the CAL data structure. The ADAG operation add labels the object as 1 (default label) and sets its default weight as 0 .

Context Dag::create (object : T) : Boolean

pre: self-->isdag()

body: self-->add(T)

post: result $=$ self $-->$ includes $(T)$

\subsection{2 delete}

This action deletes an object from the CAL data structure, relabels the ADAG (only the ancestors of the object) and resets the weight (only the ancestors of the object).

Context Dag::delete (object : T) : Boolean

pre: self-->isdag() and self-->includes(T)

body: if (self.descendants $(T)-->$ size ()$=0$ )

then 
self-->setweight(null, T)

and self-->relabel(T)

and self-- $\operatorname{delete}(T)$

else

self

endif

post: result $=$ self - - $>$ excludes $(T)$

\subsection{3 link}

This action links (creates a relationship) two elements $(P=$ parent and $C=$ child $)$ in the CAL data structure, labels $P$ object and sets $P$ 's weight according to the relationship $A$ (association).

Context Dag::link (object : A, object : P, object : C) : Boolean

pre: self-- $>$ isdag() and self.includes $(P)$ and self.includes $(C)$

body: self-->setParent $(P, C)$ and self-->setweight $(A, P)$

$$
\text { and self- }-\operatorname{relabel}(P)
$$

post: result $=$ self $->$ depend $(P, C)$

\subsection{4 unlink}

This action unlinks (deletes the relationship) two elements $(P=$ parent and $C=$ child) in the CAL data structure, labels the $P$ object and sets $P$ 's weight. 
Context Dag::unlink (object : P, object : C) : Boolean

pre: self-->isdag() and self.includes $(P)$ and self.includes $(C)$

body: if self-->depend $(P, C)$

then

self--sremoveLink $(P, C)$

and self- ->setweight(null, $P$ )

and self- $->\operatorname{relabel}(P)$

else

self

endif

post: result $=\sim$ self $-->\operatorname{depend}(P, C)$

\subsection{5 addattr}

This action adds an attribute $A$ to object $C$ in the CAL data structure.

Context Dag::addattr(object : A, object : C) : Boolean

pre: self-->isdag() and self.includes(C)

post: result $=$ self $->>$ setAttribute $(A, C)$

\subsection{Usage}

This section describes the usage of all the CAL actions. Figure 3.2 shows a sample CAL file. The model used is of the CAL parser designed and implemented as part of this thesis. The CAL parser's UML Class Diagram is shown in Appendix D and its 
XMI file, with details of classes and their relationships, is shown in Appendix F. The same model is used as an example in the case study described in Section 6.4.

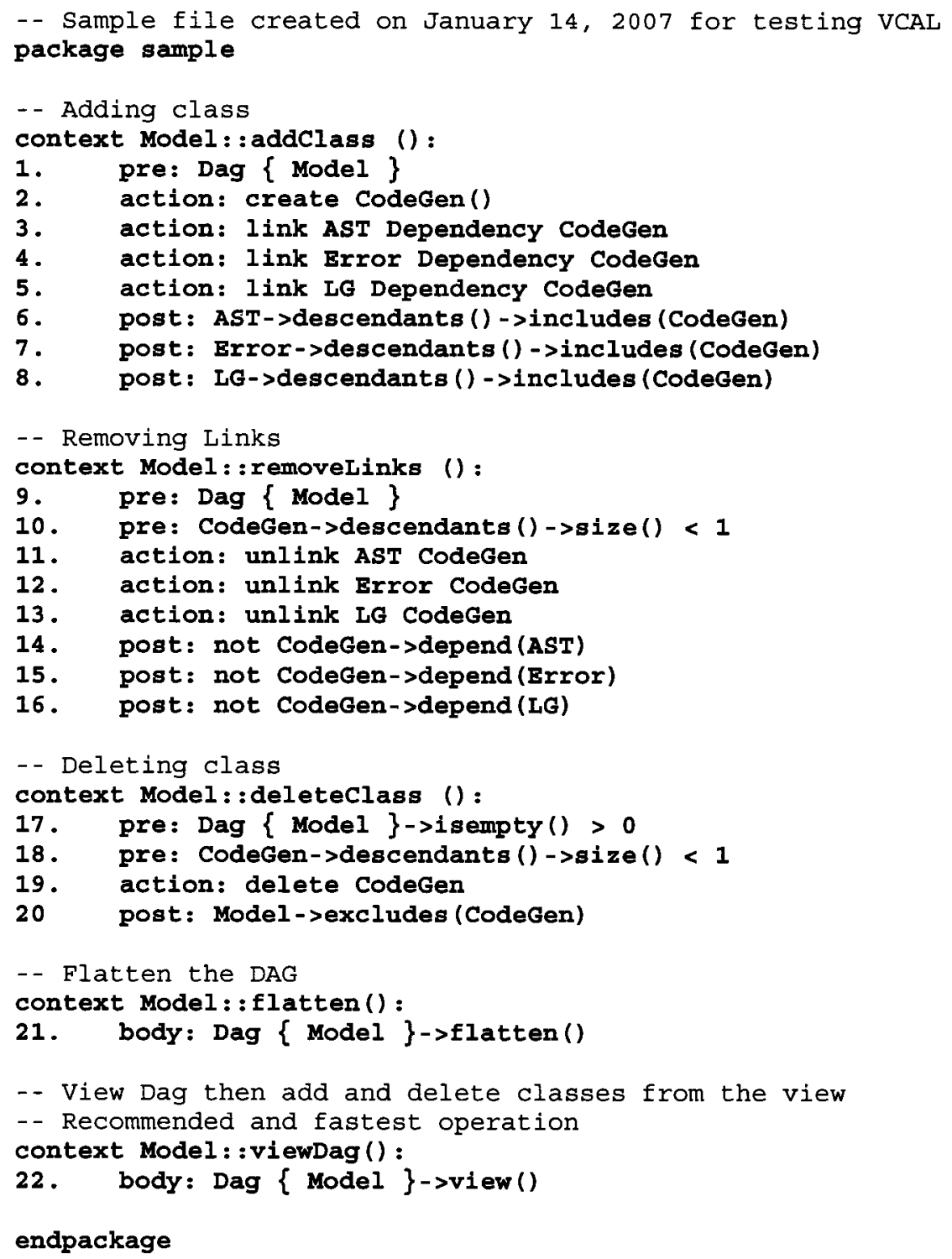

Figure 3.2 Sample CAL file

This sample CAL file shows how to use all the $5 \mathrm{CAL}$ actions defined in Section 3.3. There are 5 operation contexts listed in this CAL file. The first operation context adds a class to the model. First we create the ADAG of the model in line 1 (using a pre 
condition, i.e. the type is a DagType). In line 2, we create (add) a class CodeGen in the model. Links are created from classes $A S T$, Error, $L G$ to CodeGen class in lines 3, 4 and 5 respectively. All these links are type Dependency. Weights of these classes are set by the CAL interpreter according to this link type. In lines 6, 7 and 8 , using post conditions we check if class CodeGen is included in the descendants of $A S T$, Error and $L G$ classes respectively.

In the next operation context, removeLinks, we want to remove the links

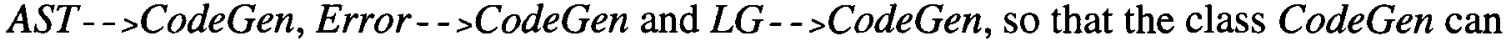
be removed later in the following operation context. Lines 11,12 and 13 remove these links. In lines 14, 15 and 16, we make sure that there is no link between class CodeGen, and $A S T$, Error and $L G$ classes.

The operation context deleteClass deletes the class CodeGen. In the pre conditions in lines 17 and 18, we check if the ADAG is not empty and there is no descendant of class CodeGen. The class is deleted in line 19. In post condition at line 20, we check if class CodeGen is not in the model.

The next operation context is simply to show how to use library operation flatten of the ADAG. It returns a tuple of 3 containing the set of all the vertices, two sets of weight and label of each vertex in the ADAG.

The last operation context is the most efficient way of using CAL actions. It creates an interactive view of the ADAG with all its vertices and edges. All 5 CAL actions can then be performed from this interactive view.

\subsection{CAL and other action languages}

This section discusses and compares action languages and their support for impact 
analysis. It is outside the scope of this thesis to cover all such action languages. Only those that are related to software models at a higher level of abstraction and specifically used for model driven software engineering (MDSE) are discussed. We have not included the action languages discussed in [16], as they have more or less the same characteristics as the action languages discussed in this thesis, and as they are also not currently used in any industrial or academic settings. Table 3.1 gives a comparison of CAL with other action languages that are mentioned in this thesis and are described below.

OAL (object action language) [24] is an action language that is fully supported by the Nucleus BridgePoint UML Development Suite from Mentor Graphics (www.mentor.com), a company specializing in electronic design automation (EDA) tools. OAL is based on action semantics (AS) [27] specifications of UML 1.5, which is a separate specification document and was not part of UML 1.5. Starting from UML 2.0, the AS specifications are part of the UML 2.0 superstructure [2]. OAL was originally designed for the models used with the BridgePoint UML Development Suite. OAL is being used for the execution and transformation of UML models and is implemented in Mentor Graphic's BridgePoint UML Suite.

ASL (action specification language) [25] is an action language also based on AS, which is now part of the UML 2.0 superstructure as described above. It is designed and implemented to work with iUML the executable UML modeling CASE tool from Kennedy Carter Limited (www.kc.com). In terms of functionalities it is more powerful than OAL. It supports complex data structures and creation of new objects (see Table 
PAL (platform independent action language) [26] is an action language that also supports the exectuable UML and is part of the PathFinder's (www.pathfindermda.com) PathMate transformation engine which is part of their PathMate Model Automation and Transformation Environment. PAL is also based on AS and supports complex data structures. The syntax of PAL is more like $\mathrm{C}++$.

Now we make a comparative analysis of these three action languages with CAL in Table 3.1. Table 3.1 does not give an extensive comparison of these languages, which is not the purpose of this thesis. The rationale for the comparison of these languages with CAL is that they are based on actions and they are capable of manipulating (changing) a UML model. We would like to see if they provide any support for impact analysis or for using constraints. We would also like to explore the potential and advantages of CAL for use as an action language. The main purpose of these action languages is to make UML models executable. Unlike OCL they can be used to make changes, but these changes do not affect the model, because, except for ASL, they can only instantiate an object but cannot create a new object in the model. They can also be used to navigate the model, just like OCL.

These action languages are designed with the design part of the software life cycle in mind, and hence they lack software maintenance capabilities (impact analysis, propagation of changes, refactoring etc.). Code can be generated in different languages like Java or C++ from UML models, or the models can be directly executed on a specific 
platform. They all are similar to OCL, but their syntax is quite different from OCL. CAL completely supports OCL concrete syntax (an OMG standard). All of these action languages support AS and AS does not have any concrete syntax, so each language has its own syntax.

These action languages are more powerful than CAL in manipulating the models; however, CAL is an academic effort and its purpose is to prove a concept, which is very new to the models and which none of them supports. Moreover, CAL can be extended to accommodate all that is offered by these languages. The main question that comes to mind here is why we need another language with a different syntax than OCL to manipulate models. Why not extend OCL, add actions like CAL and you obtain the same powerful language at a higher level of abstraction to manipulate models? They use AS but are not based on any industrial standard, so they are not interoperable between different tools. It is better and more advantageous to learn a language that is an extension of OCL, which is an industrial standard, that can support the same functionalities as these action languages.

From Table 3.1 it is clear that these languages are quite different from OCL. One of the purposes of defining a high level modeling language (UML, OCL) is that it can be used by programmers and non-programmers because it provides the same understanding of the design. The three action languages mentioned above are meant for skilled programmers. For example, the syntax for defining a structure in these languages is like a high level language (PAL's syntax is more like C++). In CAL it is more like OCL, and even simpler than OCL for a complex task of impact analysis, therefore using CAL, both programmers and non-programmers can understand and discuss the model with each 
Table 3.1 Comparison of CAL with other action languages

\begin{tabular}{|c|c|c|c|c|}
\hline Feature & CAL & OAL & ASL & PAL \\
\hline Standard Supported & $\begin{array}{l}\text { OCL } 2.0 \text { and based } \\
\text { on Action } \\
\text { Semantics of UML }\end{array}$ & $\begin{array}{l}\text { Based on Action } \\
\text { Semantics of UML }\end{array}$ & $\begin{array}{l}\text { Based on Action } \\
\text { Semantics of UML }\end{array}$ & $\begin{array}{l}\text { Based on Action } \\
\text { Semantics of UML }\end{array}$ \\
\hline Executable UML & $\begin{array}{l}1 \text { Partially } \\
\text { supported }\end{array}$ & Supported & Supported & Supported \\
\hline Impact Analysis & Supported & Not supported & Not supported & Not supported \\
\hline Extendable & Yes & Yes & Yes & Yes \\
\hline Control Statements & $\begin{array}{l}\text { If-then-else-endif } \\
\text { (Same as OCL) }\end{array}$ & if-elif-else-end if & $\begin{array}{l}\text { If-then-else-endif } \\
\text { and } \\
\text { switch-case- } \\
\text { endswitch }\end{array}$ & if-else if-else \\
\hline Loops & iterate & $\begin{array}{l}\text { for-each-end for } \\
\text { and } \\
\text { while-end while }\end{array}$ & $\begin{array}{l}\text { for-do-endfor } \\
\text { and } \\
\text { loop-endloop }\end{array}$ & $\begin{array}{l}\text { for-each-where } \\
\text { and } \\
\text { while }\end{array}$ \\
\hline $\begin{array}{l}\text { Data Structure } \\
\text { Supported }\end{array}$ & $\begin{array}{l}\text { All OCL } 2 \\
\text { Collections and the } \\
3 \text { ADAG (for impact } \\
\text { analysis) }\end{array}$ & Not supported & $\begin{array}{l}6 \text { Set with } \\
\text { hierarchical data } \\
\text { support }\end{array}$ & $\begin{array}{l}\text { Group (ordered set } \\
\text { of items) and } \\
\text { GroupIter (iterator } \\
\text { over Group) }\end{array}$ \\
\hline Navigation & Yes & Yes & Yes & Yes \\
\hline Communication & Messages (OCL) & Events & Signals & Events \\
\hline Operators & Same as OCL & $\begin{array}{l}\text { Almost same as } \\
\text { OCL }\end{array}$ & $\begin{array}{l}\text { Almost same as } \\
\text { OCL }\end{array}$ & $\begin{array}{l}\text { Almost same as } \\
\text { OCL }\end{array}$ \\
\hline Actions & $\begin{array}{l}\text { Objects can be } \\
\text { created and added } \\
\text { but cannot be } \\
\text { instantiated }\end{array}$ & $\begin{array}{l}\text { Objects cannot be } \\
\text { created or added } \\
\text { but can be } \\
\text { instantiated }\end{array}$ & $\begin{array}{l}\text { Objects can be } \\
\text { created, added and } \\
\text { can also be } \\
\text { instantiated }\end{array}$ & $\begin{array}{l}\text { Objects cannot be } \\
\text { created or added but } \\
\text { can be instantiated }\end{array}$ \\
\hline Timers & Not supported & $\begin{array}{l}4 \text { External timer } \\
\text { and } 5 \text { internal timer } \\
\text { supported }\end{array}$ & $\begin{array}{l}\text { Relative and } \\
\text { absolute timer }\end{array}$ & Not supported \\
\hline Tool support & $\begin{array}{l}\text { An interoperable } \\
\text { prototype tool has } \\
\text { been implemented } \\
\text { as part of the } \\
\text { research }\end{array}$ & $\begin{array}{l}\text { BridgePoint UML } \\
\text { Suite ® by Mentor } \\
\text { Graphics }\end{array}$ & $\begin{array}{l}\text { CASE tool iUML } \\
\text { ® by Kennedy } \\
\text { Carter Limited }\end{array}$ & $\begin{array}{l}\text { PathMate } \\
\text { Transformation } \\
\text { Engine ® by } \\
\text { PathFinder Solutions }\end{array}$ \\
\hline $\begin{array}{l}\text { Executable } \\
\text { executable n } \\
\text { Example of } \\
\text { Read chapte } \\
\text { Time extern } \\
\text { The internal } \\
\text { Syntax for d } \\
\text { defi }\end{array}$ & $\begin{array}{l}\text { UML as a model is su } \\
\text { model / platform is no } \\
\text { defining one (the Set) } \\
\text { r } 4 \text { for more details } \\
\text { al to the application. } \\
\text { 1 system clock that me } \\
\text { defining the set in ASI } \\
\text { fine structure <struct } \\
\quad<\text { member name } \\
<\text { member name } \\
\text { ddefine }\end{array}$ & $\begin{array}{l}\text { ipported for manipulat } \\
\text { of the collections in } \\
\text { For example October } \\
\text { asures the time in "tic } \\
\text { ire type name }> \\
><\text { member type }> \\
><\text { <member type }>\end{array}$ & $\begin{array}{l}\text { tion, but the generatio } \\
\text { OCL: Set }(2,4,1,5 \text {, } \\
14,2006 \text {. } \\
\text { ks" for supporting re }\end{array}$ & $\begin{array}{l}\text { nof code or } \\
7,13,11,17\} \\
\text { l-time systems. }\end{array}$ \\
\hline
\end{tabular}


other, which greatly reduces the time and cost of completing any software system or software project. 


\section{Chapter 4}

\section{DAG for Dependency Analysis}

There are various applications for a directed acyclic graph (DAG) in computer science and software engineering. They are used in compilers to generate a parse tree with parent and child relations and to schedule instructions. They are used to represent networks and hierarchical systems of folders in an operating system, and relationships between classes and subsumption hierarchies of data available on the semantic web, such as Netscape Open Directory (ODP). In object oriented graphics the scene graphs are stored as a DAG. Whenever an operation is applied (in the scene graphs) to a parent node or nodes, it automatically propagates its effect to all the children. To date, ODP has indexed over 4 million sites (source: http://directory.mozilla.org) and is being used by large search engines like Netscape, AOL and Google.

Because of the number of important applications of a DAG, a great amount of effort $[36,38,39,40,42,43]$ has been put into labeling these graphs to improve typical operations on a DAG. In this chapter we show how a labeling scheme, DAG-Lite [40], has been used and improved in size (i.e., the number of digits in a label and the size of all the labels), and a dependency relationship weight (DRW) has been added to the DAG. We use this weight (DRW) for computing the dependency or relationship weight of each vertex of the DAG with its descendants. We call this an annotated DAG (ADAG), and it is used as the CAL (constraints with action language) data structure. Dependency 
analysis involves examining detailed dependency relationships among program entities [7] and is one of the methods used in impact analysis. Each vertex in the ADAG is an entity and an edge represents the dependency relationship between two entities (vertices). The improved labeling scheme and the weight are used to effectively display, visually, this dependency analysis. The assignment and computation of the weight of each node are described in Section 5.2.2.3 and are formally defined as Rule 1.5 in Chapter 5 and Equation 3.1 in Chapter 3 respectively. In this chapter, we describe the improved labeling scheme using mathematical equations and present efficient algorithms for labeling and performing typical operations for dependency analysis on the ADAG.

A prototype tool VCAL (visual CAL) has been implemented to display, visually, the ADAG. VCAL and its implementation will be described in Chapter 6. All the ADAG operations are defined in OCL syntax in Chapter 3. The ADAG operations that are defined and described as algorithms in this chapter are relabel(node), label(node), descendants(node), contains(node), depend(node1, node2), setParent(parent, child), and removeLink(parent, child). The important characteristics that are required in a labeling scheme, and operations and queries on the ADAG that are required for graphical representation of dependency analysis are summarized as follows:

- Size of the label. It should be as compact as possible.

- Time it takes for the queries to complete for effective graphical representation of dependency analysis.

- Updates should require minimal relabeling.

- Reachability: Checks if one of the vertices of the ADAG is reachable from other vertices, i.e. if a path exists between two vertices. For any two vertices $x$ 
and $y$ in the graph we define it as a directed path from $x$ to $y$ and represent it as follows:

$x-->$ y. For example in Figure 4.1 we can write $P-->F$ is true.

- Descendants: Find all the descendants of a vertex, i.e. which of other vertices depend on the vertex. If $V$ is a set containing all the vertices of the ADAG, then $D(x)$ is a subset of $V$ containing all the dependent vertices (descendants) of any vertex $x$ in the ADAG. For example in Figure 4.1 we can write $D(L)=$ $\{H, D, E\}$.

Vertices $A, B, C, D, E$ and $M$ in Figure 4.1 are leaves and have no descendants.

For the purpose of better comprehension and description of the algorithms in this chapter we assume that the ADAG is stored in a hash table (that is how the ADAG has been implemented in VCAL). We call this hash table $D A G_{-} H T$. We also assume that $D A G \_H T$ uses a hash(node) function to compute the hash of the node (node and vertex are same) for storing the node in the hash table. The global variable size is used to keep and set the size of the ADAG. The actual data structure for a node (a Java Class) in VCAL is defined as follows, and we use the same data structure in describing the algorithms in this chapter:

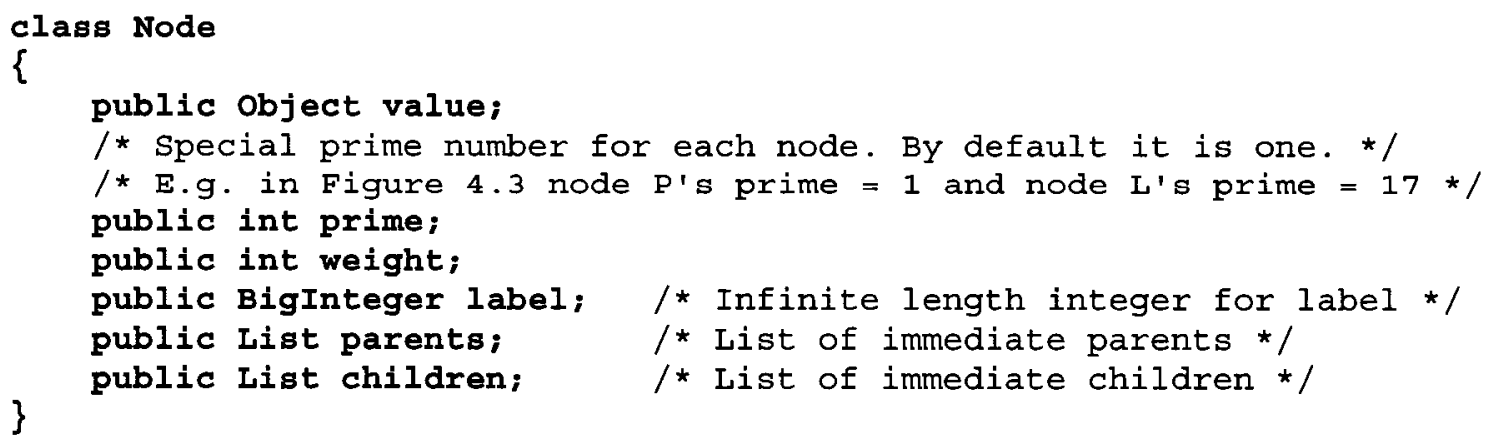




\subsection{DAG Labeling}

A directed graph or digraph consists of a set of vertices and a collection of ordered pairs of distinct edges. If the ordered pair $(x, y)$ is an edge $e$ then we say that the edge $e$ is directed from $x$ to $y$. If there are no directed cycles (i.e. for any vertex $x$ there is no directed path starting and ending on $x$ ) in a digraph, then it is called a DAG. Figure 4.1 shows a sample ADAG that we use to explain the scheme for labeling the graph in this chapter. We formally define an ADAG $G$ (the same definition is given for $D$ in Section 3.2.2) as follows, where $V$ is the set of all the vertices, $E$ the set of all the edges, $L$ the set of all the labels and $W$ the set of all the weights:

$$
G=(V, E, L, W)
$$

where

$$
E=(x, y)=\{x y: x \in V, y \in V, x y \in E\}
$$

such that $E$ is a Partial order (transitive and non-reflexive) on set $V$

$L=\{(l(x) \cup l(y)) \in L: x y \in E\}$

$l(x)$ is the label of $x$ and $l(y)$ is the label of $y$

$W=\{w(x, y) \in W: x y \in E\}$

$w(x, y)$ is the weight of $x$ that is based on the relation $x \rightarrow y$

The indegree of a vertex is defined as the number of edges adjacent to that vertex, and outdegree is defined as the number of edges adjacent from a vertex.

Ideally, for any two vertices we should be able to determine reachability between them by comparing only their labels. For the graph in Figure 4.1 with 17 vertices, how can we determine

$$
\text { if } N-->D \text { or } N-\longrightarrow>A \text { ? }
$$




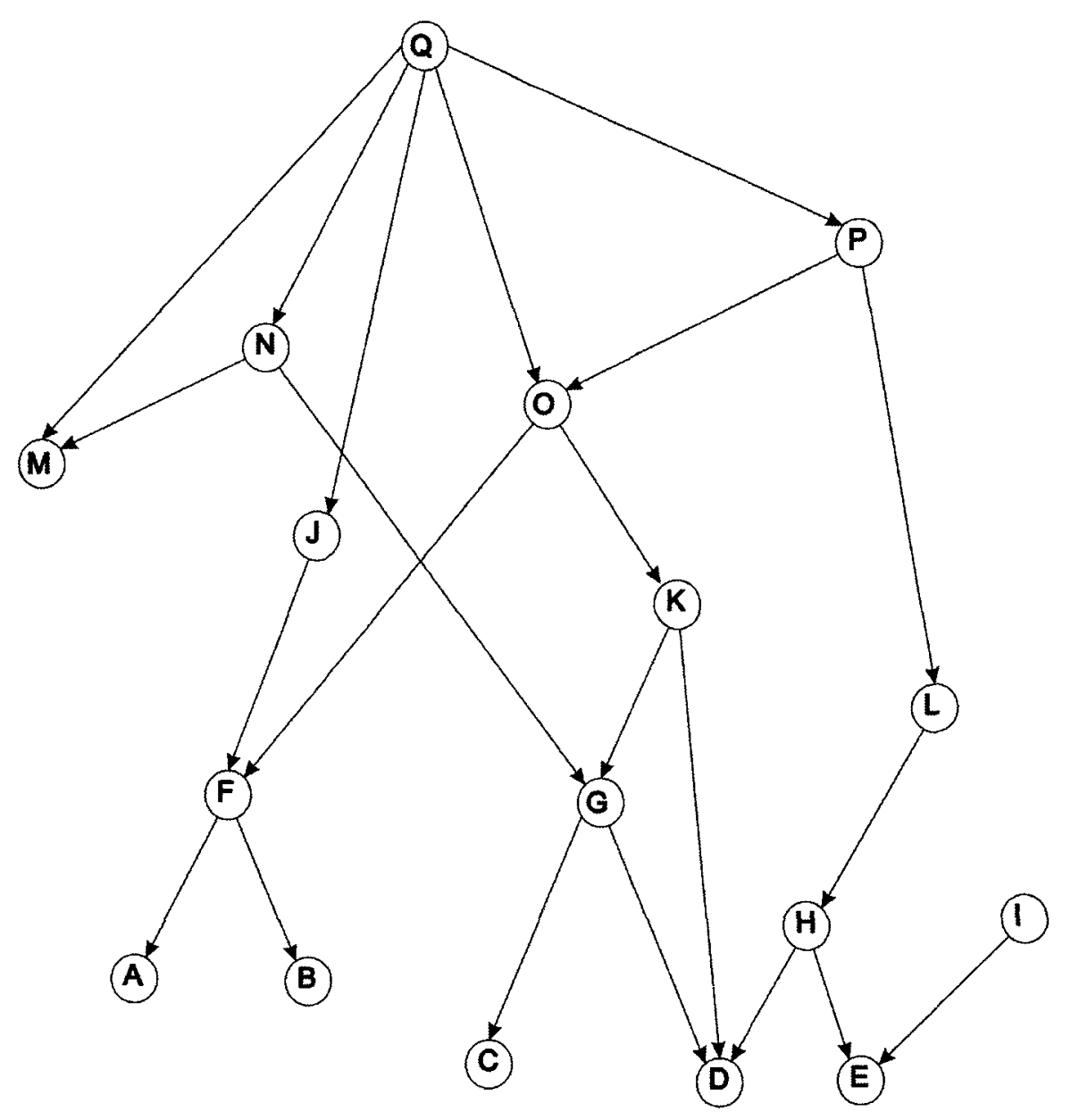

Figure 4.1 Sample DAG

By inspecting the DAG and tracing the paths, we can determine that the first statement is TRUE and the second statement is FALSE. However, for a graph of more than 100 vertices it becomes, if not impossible, difficult to visually determine reachability between two distant vertices. First, it is difficult to visualize such a graph and if we can, then it takes more time and work to find the reachability and descendants of all the vertices, which is required for dependency analysis. If we compute reachability ideally as defined above, then we can achieve an efficient automation and graphical representation of the DAG for dependency analysis. No matter how complex or deep a 
DAG is, the scheme presented in this chapter labels the DAG in such a way that a graphical representation tool can compare the vertices for dependency (i.e. reachability and descendants) by simply clicking (one-click) on the vertices, as is done in VCAL. Using this labeling scheme, VCAL applies basic filters to the ADAG and achieves oneclick dependency analysis. The next section presents and describes this scheme.

\subsubsection{Labeling scheme}

We have used one of the properties of prime numbers, uniqueness of prime factorization, for labeling the ADAG, which can be defined as any integer $n \in N$ can be factorized into unique prime numbers, and we can write

$$
n=\prod_{i=1}^{f} p_{i},
$$

where $p$ is a prime number and $f \geq 1$. Hence, an integer $n$ can be divided by any of its factors, and we can write

$$
n \bmod p_{i}=0
$$

Using this property of prime numbers, we label all the vertices such that simply by knowing the labels of two vertices we can compute the reachability between the two vertices. Descendants of any vertex can also be computed by either taking mod or using all the factors of the vertex's label. These two approaches are discussed below. We also show how our approach is different and better in size than the approach used in DAGLite [40].

The equation used for labeling a DAG is shown in Figure 4.2. In Equation 4.1, $L(v)$ is only multiplied with $n p$ when its label equals any one of its children's label. This greatly improves the size of the label as compared to the label size of DAG-Lite in [40] 
with an average complexity DAG.

Let $a, b \in N$ and $L C M[a, b]$ as the least common multiple of $a$ and $b$, the set of children of $v \in V, C(v)=\{v 1, v 2, v 3, \ldots, v n\}$, where $1 \leq n \leq$ outdegree $(v)$ and $n p$ is the next prime from an ordered list of primes starting with 2 . Then we define two equations for labeling the DAG as follows:

$$
\begin{aligned}
& \operatorname{label}(v)=\mid \begin{array}{ll}
1 & \text { outdegree }(v)=0 \\
L(v) \times n p & L(v)=\left.\operatorname{label}(v i)\right|_{i=1} ^{n} \\
L(v) & \text { else }
\end{array} \\
& L(v)=L C M[\text { label }(v 1), \text { label }(v 2), \ldots, \text { label }(v n)]
\end{aligned}
$$

Figure 4.2 Equations for computing the label of a vertex in the DAG

The complete labeled DAG using the scheme in Figure 4.2 is shown in Figure 4.3. Algorithms for labeling the ADAG are listed in Figure 4.4. All the leaves, $A, B, C, D, E$ and $M$, are labeled as 1 . The prime numbers used are underlined. We can find the reachability between node $Q$ and node $I$, by computing the mod of the label of node $Q$ with the label of node $I$ as follows:

$l(Q) \bmod l(I)=1385670 \bmod 7=1$. Therefore, node $I$ is not dependent on node $Q$.

We can find all the descendants of a vertex by computing the mod of each vertex's label with the label of the vertex. If the mod is 0 then a descendant of the vertex is found and vice versa. For vertex $P$ in Figure 4.3 we find the following set, $D(P)$, of descendants of $P$ : 


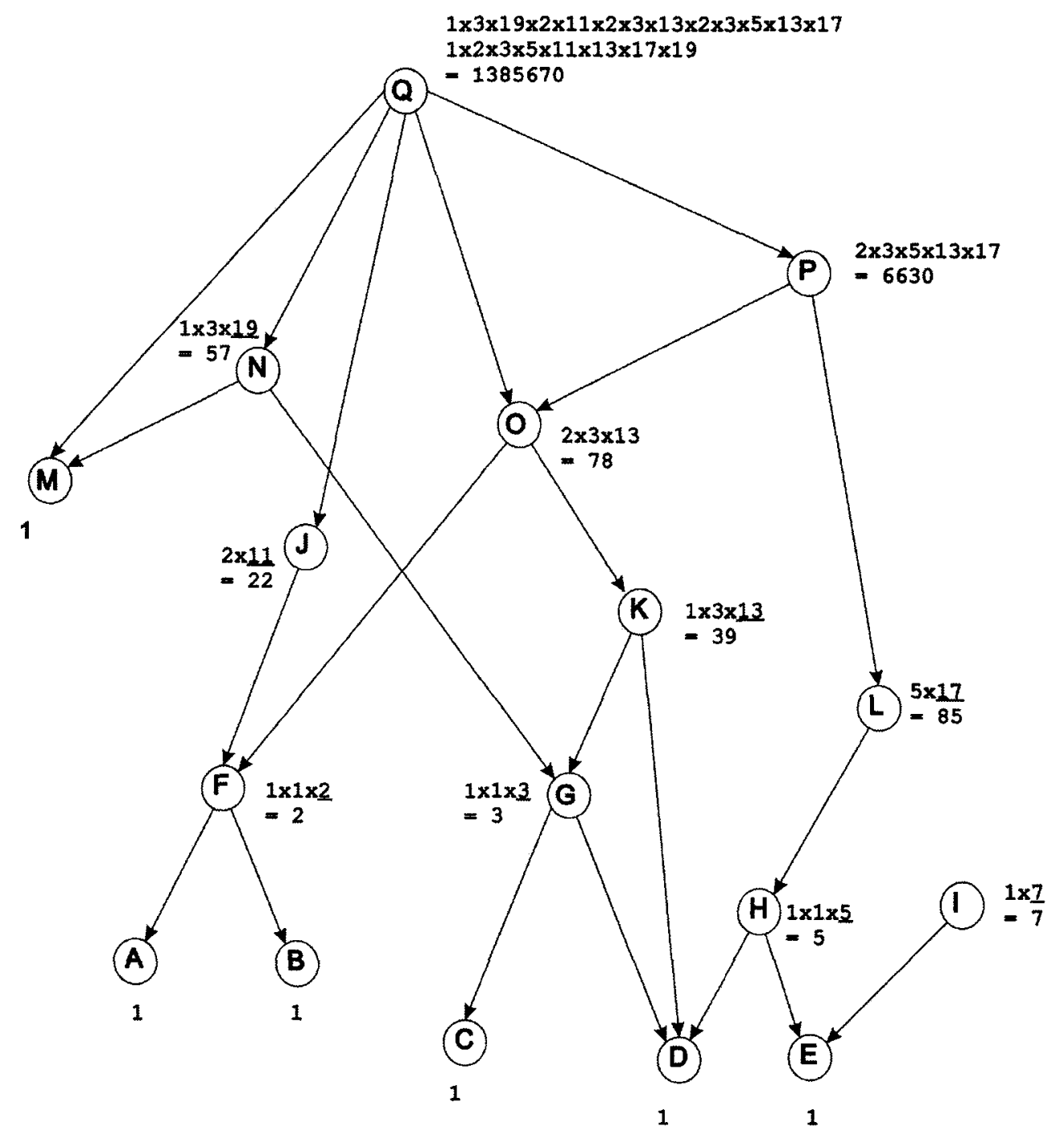

Figure 4.3 Labeled DAG

$D(P)=\{O, L, K, F, G, H, A, B, C, D, E\}$.

Since leaves in the DAG are labeled as 1, we do not use their label for finding either the reachability or the descendants. Instead, we use the label of their immediate parents (see Figures 4.5 and 4.6).

\subsubsection{Size of the label}

Since we are using prime numbers from an ordered list of primes starting from 2 , we can calculate the maximum number $M$ that can be used for a label in the DAG using 68 
the following equation:

$$
M=\prod_{i=2}^{m} p i
$$

where $m$ is the maximum prime number used and $p$ is the prime factor of $m$

The size of $M$ depends on $m$. For a DAG with $n$ vertices, $m$ can be calculated for worst and average cases using Equation 4.1 as follows:

Worst case $\boldsymbol{m}=\boldsymbol{n}-\boldsymbol{1}$ : When a DAG is a straight line, then outdegree $(v)>0$, and initially the label of each vertex is equal to its child's label so each vertex is multiplied by $n p$.

Average case $\boldsymbol{m}=\boldsymbol{n}$ / 2: An example is a DAG with average complexity. In Figure 4.3 the underlined primes are the only primes used.

Not every vertex in the DAG is multiplied by an exclusive prime number. In Equation (1), $L(v)$ is only multiplied by $n p$, when it equals one of the labels of children of $\mathrm{v}$ and hence reduces the size of $\mathrm{m}$ in Equation (3). This significantly reduces the number of digits in the label as compared to the similar scheme used in DAG-Lite, which assigns an exclusive prime number that is multiplied by the label of all the vertex's parents, as defined in Equation (1) in [22]. We have also improved the space for storing all the labels of the node in a DAG by assigning 1 as the label of leave vertices as compared to the scheme, DAG-Lite, which assigns an exclusive prime number to a leave, which is multiplied by the label of all the leave's parents.

\subsection{Labeling and dependency analysis algorithms}

The vertices can be labeled as the graph is built or with an already built graph. Based on the scheme described above we present here algorithms to relabel and compute 
the label of any node in the DAG, and to find reachability between two nodes and the descendants of any node in the DAG. Here we refer to a node as a vertex of a graph.

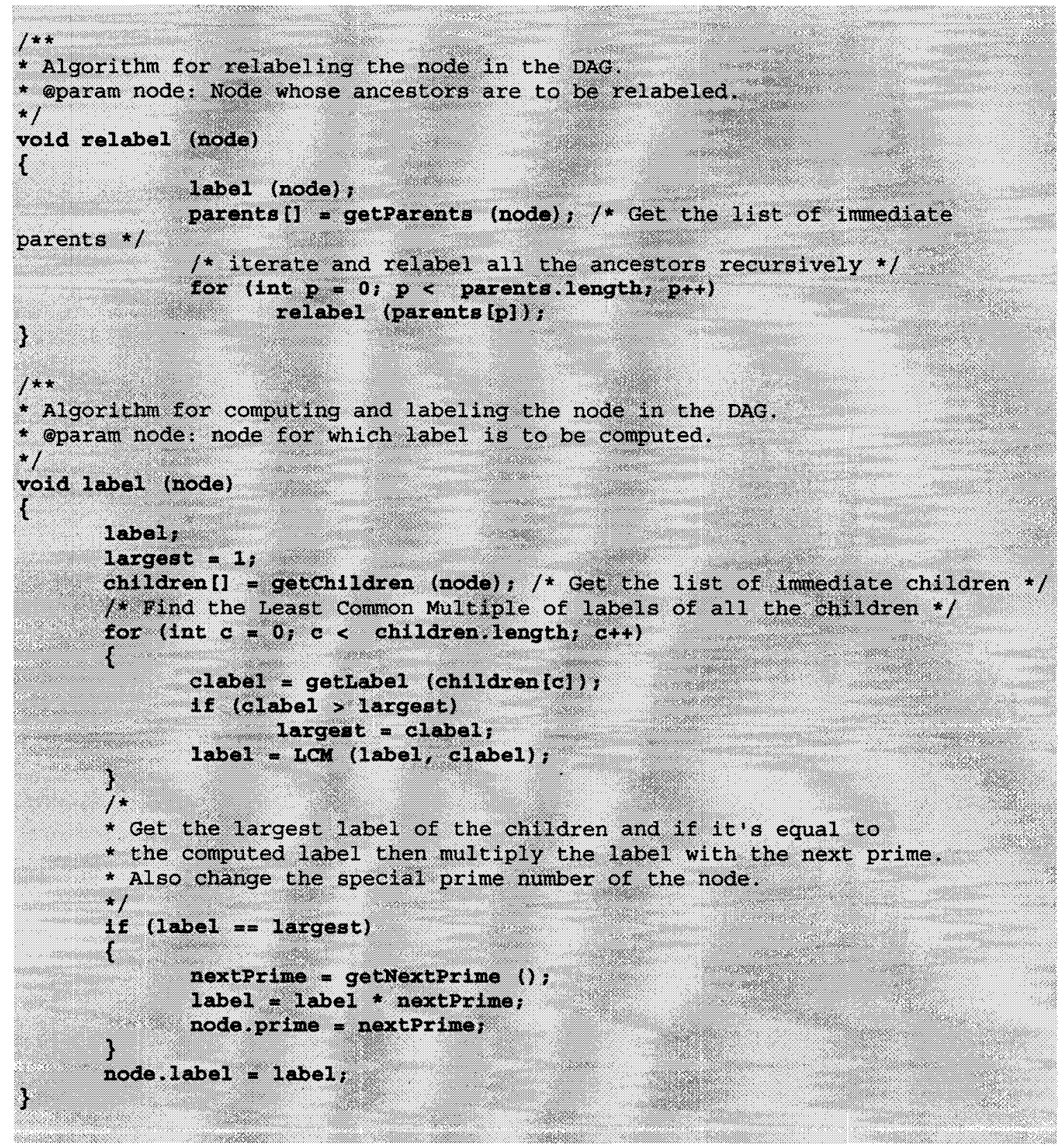

Figure 4.4 Algorithms to label a node in the DAG

Algorithms for these operations in C-like syntax are listed in Figures 4.4, 4.5 and 4.6. We also compute time complexity for 2 of these algorithms. 


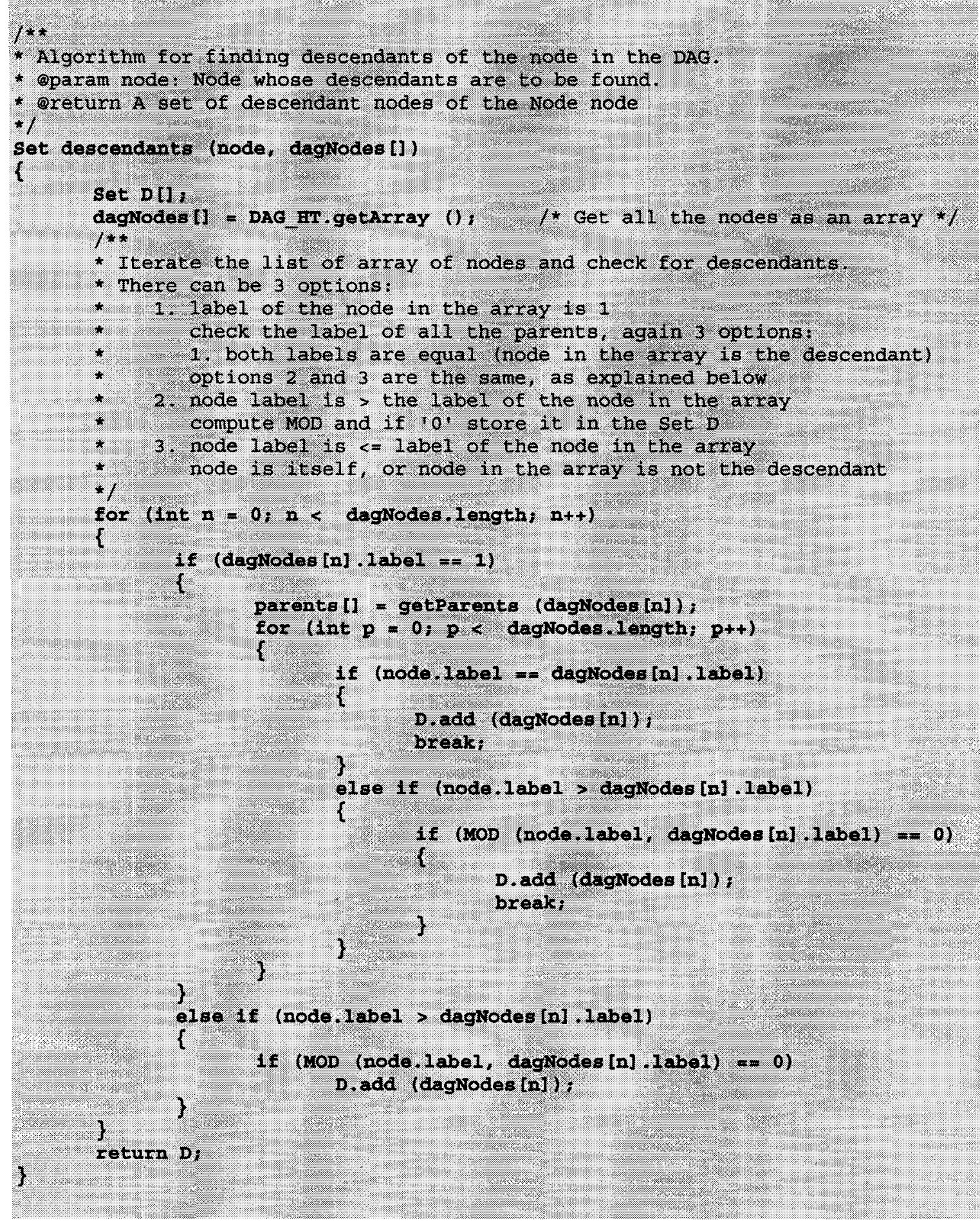

Figure 4.5 Algorithm to find descendants of a node in the DAG

\subsubsection{Time complexity}

In this section, we compute the time complexity of the algorithms to find the 
descendants of a node (see Figure 4.5) and find the reachability between two nodes (see Figure 4.6).

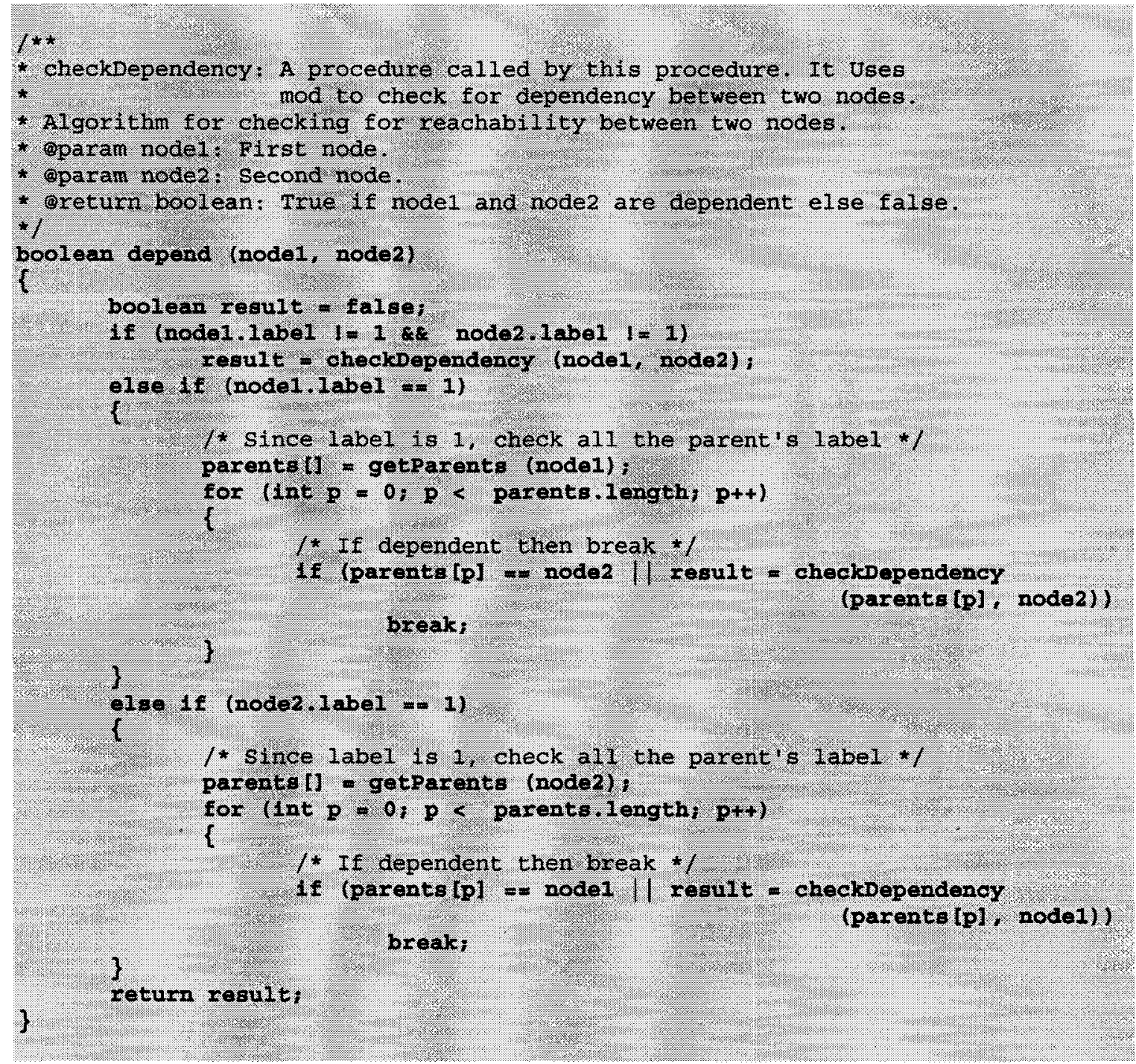

Figure 4.6 Algorithm to find reachability of two nodes in the DAG

descendants: The DAG is stored in a simple list whose length is $n$, where $n$ is the number of vertices in the DAG. The for loop to find descendants in Figure 4.5 runs for the length of the list, so the total number of operations is equal to the length of the list, which is the number of vertices in the DAG. Hence, the time complexity for this 
operation is $O(n)$, both in average and worst cases, where $\mathrm{n}$ is the number of vertices.

depend: There is no loop in the algorithm listed in Figure 4.6 (MOD is a mathematical operation without any loop), so the time complexity for this operation is $O(1)$ in an average case and $O(n)$ in a worst case, when the label of a node is 1 and the node's immediate parents is equal to all the nodes in the DAG, where $\mathrm{n}$ is the number of vertices.

\subsection{Updates}

To add or delete a node, only the ancestors are relabeled, as shown in Figures 4.10 and 4.11. When node $R$ is added in Figure 4.10, only node $Q$ is relabeled, which in this

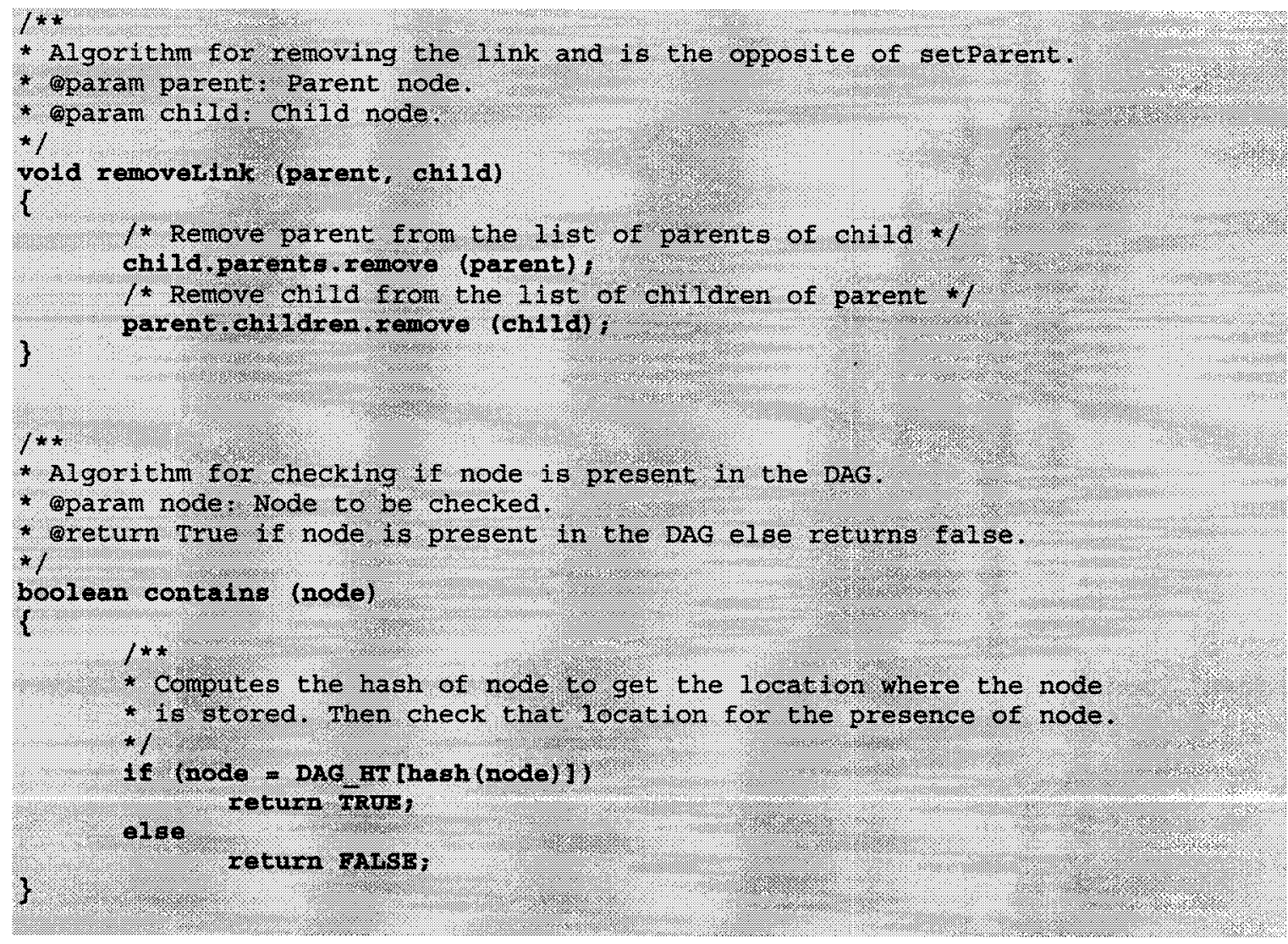

Figure 4.7 Algorithms to remove the link of a node from the DAG, and check if a node is present or not in the DAG 
case does not change the label of the node $Q$. When the node $K$ is removed in Figure 4.11, only nodes $O, R$ and $Q$ are relabeled. Algorithms for these update operations are listed in Figure 4.9. We also present algorithms for three other operations (setParent, removeLink and contains - see Figures 4.7 and 4.8) for the DAG, which are used by the CAL actions (see Section 3.2).

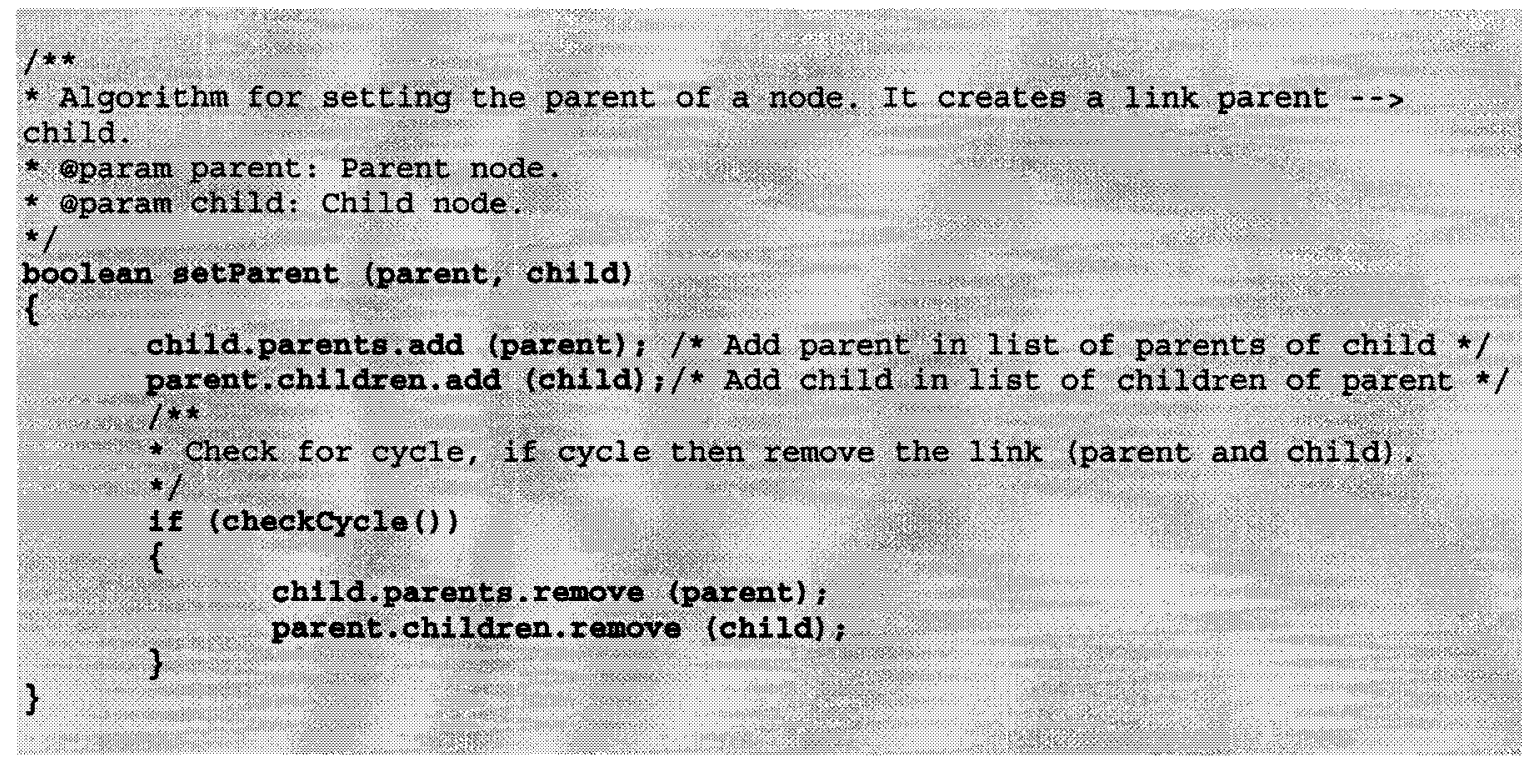

Figure 4.8 Algorithm to set the parent of a node in the DAG

\subsection{Related work}

An earlier scheme [36] by R. Khatib and N. Santoro labeled an acyclic graph using a scheme based on a minimum-distance spanning tree using post-order traversal, for optimizing routing in communication networks. There are three popular labeling schemes for graphs discussed in [38]. The first one is the bit-vector scheme in which, as the name suggests, a node is represented by a vector of bits. A 1 bit at some position identifies the node in a lattice. Each node inherits the bits identifying its ancestors or descendants in a top-down or bottom-up encoding. The second scheme is the prefix 
scheme, which encodes the parent directly in the label of the node as a prefix using any tree-traversing technique. The third one is the interval scheme in which the node of the

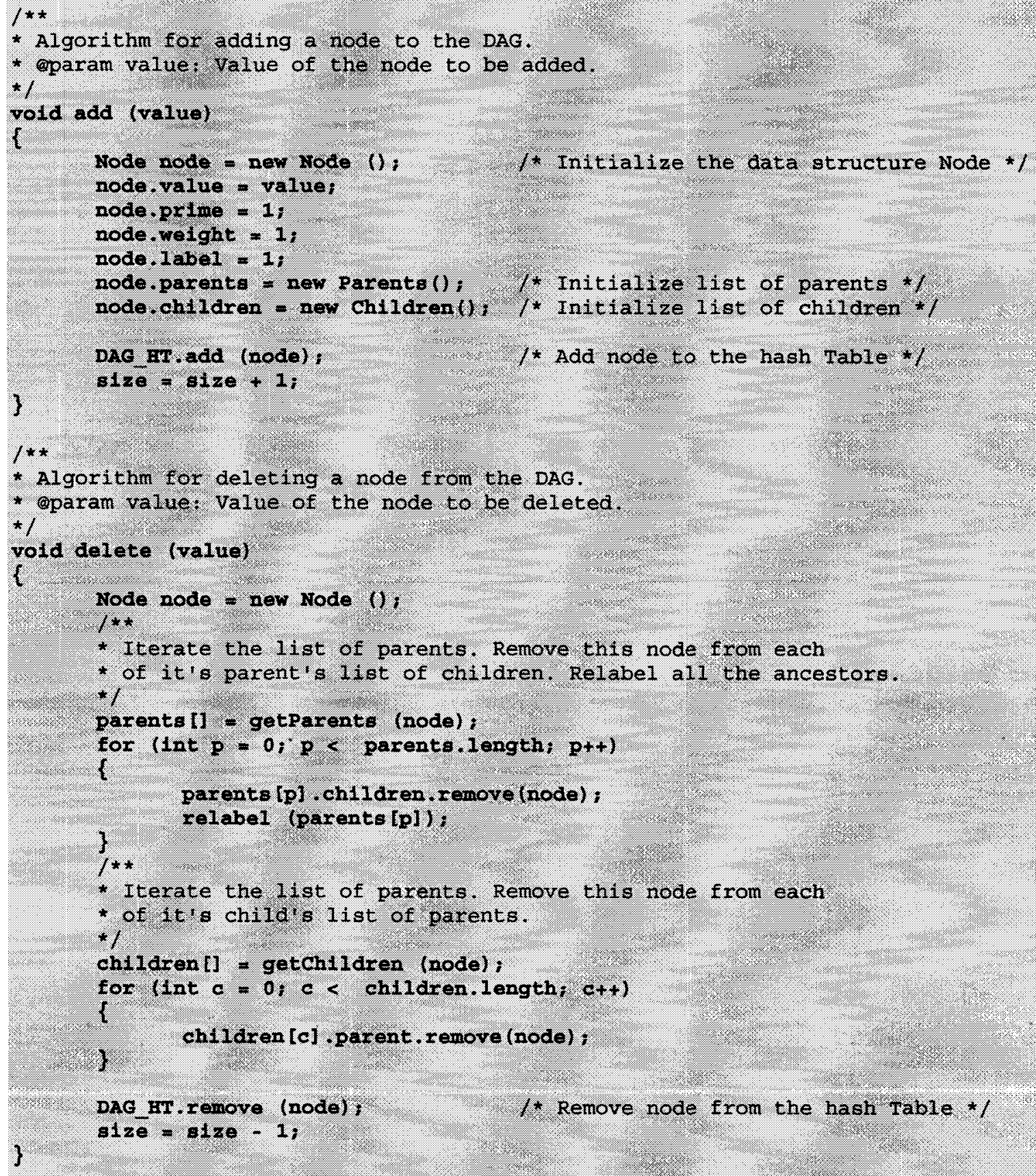

Figure 4.9 Algorithms to add and delete a node in the DAG

label is given by an interval, such that it is contained in its parent's label. The last two 
schemes discussed above are based on spanning trees.

A recent scheme that is not based on spanning trees is 2-hop labels [39]. This scheme uses hops (distance between vertices) to label the vertices. The label gives the distance between vertices. This distance can be used for dependency analysis and can be mapped to the dependency between two or more than two vertices. In future research, this distance could be combined with the weights used in this thesis for dependency analysis.

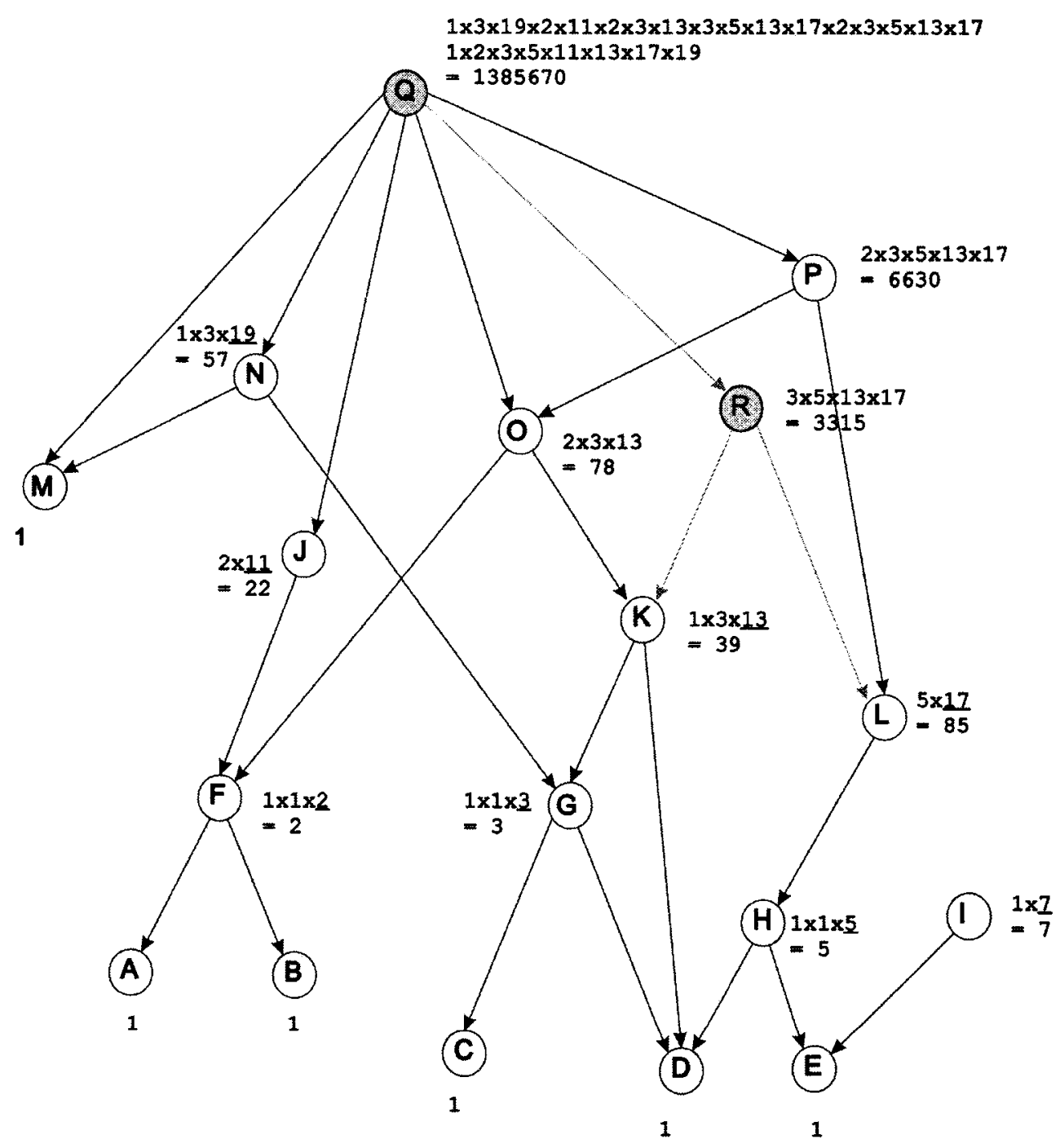

Figure 4.10 Labeled DAG with node $R$ added 
Recent labeling schemes $[42,43]$ have proposed and applied labeling schemes for tree structured XML data. In [42] a DAG labeling scheme is applied to XML data. Before applying this scheme the XML data is modelled as a directed cyclic graph and then reduced to a DAG. After labeling the DAG using the interval scheme described above, is again converted back to a directed cyclic graph. The scheme proposed in [43] is an improvement on the prefix scheme described above. A detailed survey of labeling schemes can be found in [37].

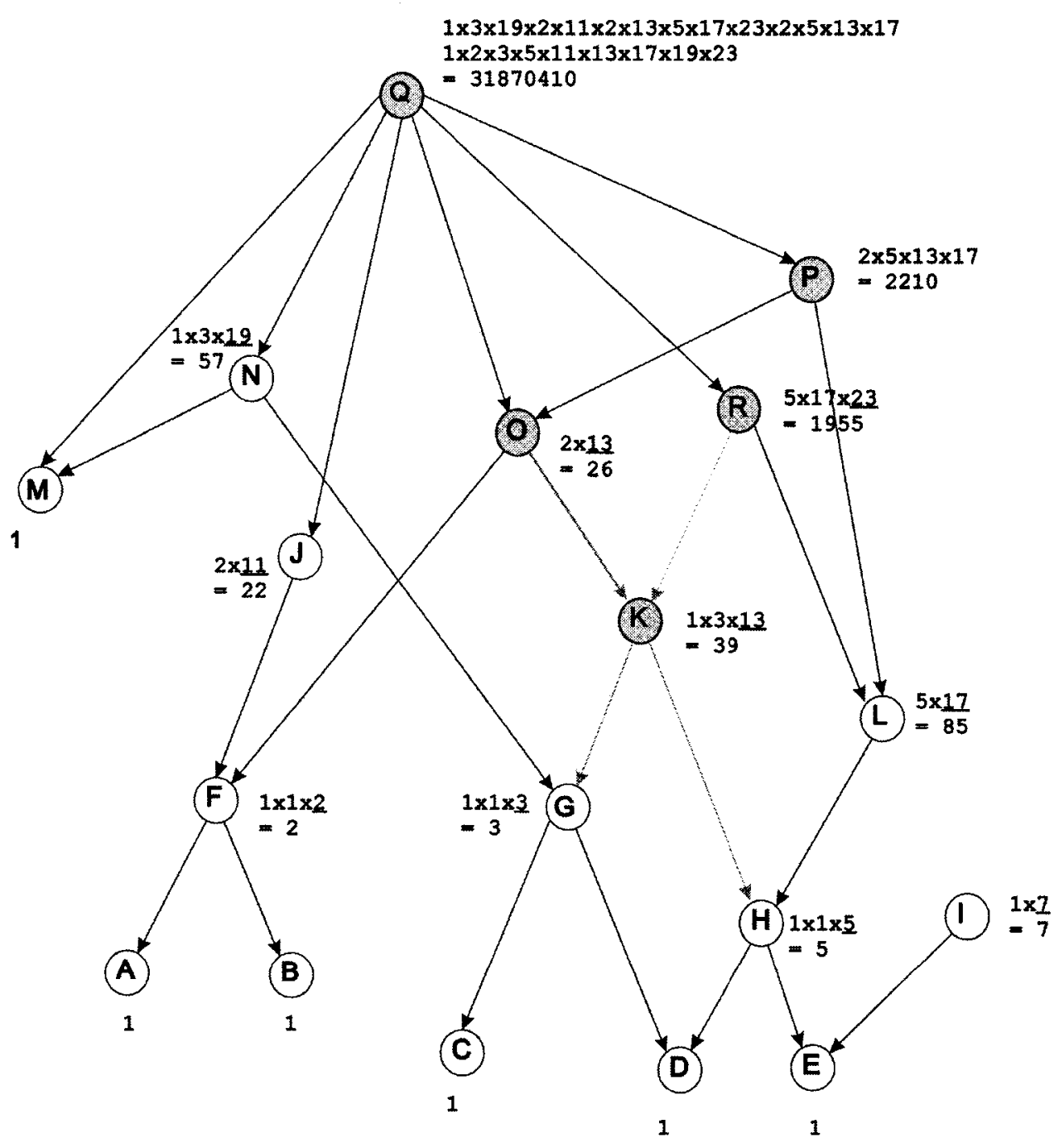

Figure 4.11 Labeled DAG with node K removed 
Currently prime numbers are, being used for labeling an XML tree [41] and a DAG [40]. This improves operations like reachability and descendants. In this thesis we have used and improved in size the labeling scheme DAG-Lite [40] for labeling a DAG. 


\section{Chapter 5}

\section{CAL Implementation}

Constraints with Action Language (CAL) allows a user to perform different queries and tasks on models without knowledge of the complexity of the rule execution engine that processes and translates these models, so that these queries and tasks can be interpreted and executed by the CAL interpreter. However, a tool that implements CAL for UML models needs to parse and process these rules. In this chapter, we formally specify and explain the rules for translating a UML Class Model to the CAL data structure (ADAG) and the execution of CAL operations and actions (CAL model). The detailed implementation of the tool that implements the rule execution engine will be explained in Chapter 6. There are two kinds of rules that this engine processes: one for translating UML Class Model to the CAL data structure (ADAG) and the other for executing CAL operations and actions. CAL operations and actions are specified using TLA+ [50] for verification.

\subsection{Rules for translating a UML Class Model to the CAL data structure}

Before specifying these rules we first formally define a graph. A simple graph is represented by a tuple of two, $\mathrm{V}$ the set of all the vertices, and $\mathrm{E}$ the set of all the edges. The graph $\mathrm{G}$ is defined as follows:

$$
\begin{aligned}
& G=(V, E) \\
& E=(x, y)=\{x y: x \in V, y \in V, x y \in E\}
\end{aligned}
$$


We extract, using pattern matching techniques, sets of all the following dependency relationships between UML Class Model elements, as defined in UML 2.0 [5] and explained in Section 2.2:
a. Dependency
b. Composition
c. Generalization
d. Realization

Let $m$ be a model element, then

R 1.1: $V_{m}\{(m \in V) \wedge \neg(\neg m \in V)\}$

If $x, y$ and $z$ are model elements, and

$R_{D} \in($ Dependency $\vee$ Composition $\vee$ Generalization $\vee$ Realization $)$, then

R 1.2: $V_{x, y}\left\{(x y \in E) \rightarrow\left(\left(x R_{D} y\right) \vee\left(y R_{D} x\right)\right)\right\}$

R 1.3: $V_{x, y}\left\{\left(x R_{D} y\right) \rightarrow \neg\left(y R_{D} x\right)\right\}$

R 1.4: $V_{x, y, z}\left\{\left(\left(x R_{D} y\right) \in\left(y R_{D} z\right)\right) \rightarrow\left(x R_{D} z\right)\right\}$

We introduce and define another tuple $W$ as the set of self-weight of all the vertices of the graph as follows:

$W=\{w(x) \in W: x \in V\}$

R 1.5: $V_{x, y}\left\{\left(x R_{D} y\right) \wedge\left(\left(R_{D} \in(\right.\right.\right.$ Dependency $\vee$ Realization $\left.)\right) \rightarrow(w(x)=1) \vee$

$$
\left.\left.\left(R_{D} \in(\text { Generalization } \vee \text { Composition })\right) \rightarrow(w(x)=2)\right)\right\}
$$

According to rule $R 1.1$ only the model elements become vertices of the graph. Rule $R 1.2$ states that if there is a relationship between two model elements $x$ and $y$ then they become an edge in the graph. Rules $R 1.3$ and $R 1.4$ describe the relationship $R_{D}$ as 80 
non-reflexive and transitive respectively, so by definition this directed graph is a DAG. Rule $R 1.5$ assigns a self-weight of 2 if the relationship $R_{D}$ between two model elements, a supplier $x$ and a client $y$, is a generalization or composition relationship, and a selfweight of 1 if the relationship $R_{D}$ between two model elements, a supplier $x$ and a client $y$, is a dependency or realization relationship. This assignment of self-weight to the model elements is due to the couplings of different relationships between the UML Class Model elements as described in UML 2.0 and shown in Table 5.1. The relationships are listed in order of coupling from high to low. The characteristics in Table 5.1 show that these relationships are transitive and non-reflexive. This further augments Rules $R 1.3$ and $R$ 1.4.

Table 5.1 Characteristics and coupling of different relationships in UML

\begin{tabular}{|l|l|l|}
\hline \multicolumn{1}{|c|}{ Relationship } & \multicolumn{1}{|c|}{ Coupling } & \multicolumn{1}{c|}{ Characteristics } \\
\hline Generalization & Functional, Data & Transitive, Non-Reflexive \\
\hline Composition & Functional, Control, Life & Transitive, Non-Reflexive \\
\hline Dependency & Functional & Non-Reflexive \\
\hline Realization & Functional & Non-Reflexive \\
\hline
\end{tabular}

\subsection{CAL model}

The CAL model consists of 10 operations and 5 actions. This section formally describes and explains 4 of these operations and 1 of the actions. There are 5 action statements defined in the CAL grammar (see Appendix A), and 10 CAL operations 
defined in Section 3.2. All the CAL actions were informally defined and explained in Section 3.3 using OCL-like syntax. Appendix E lists specifications of all the CAL operations with their helper libraries and one of the CAL actions, create, and its subactions, relabel and setweight, using TLA+ (see Section 2.6). The format used is ASCII for TLA+ as described in [50], so that it can be parsed and compiled by TLC. The operations and actions are specified as procedures using pluscal (see Section 2.6), which is based on TLA+. These specifications have been tested and verified using the TLC model checker. Since CAL operations are performed on the ADAG, we call them ADAG operations. We also calculate the compute time for the operations depend and descendants, which are used for dependency analysis.

Figure 5.1 specifies the data structure (variables) and definitions to be used in the specifications. We assume that the set of vertices (line 3) and the set of edges (line 4) already contain data, as shown in Figure 5.1. The operation isdag(), which is used in these specifications and specified in Appendix E, determines whether if the input is a DAG or not by recursively checking if there is any cycle caused by the input set, edge. This operation uses the set parents (line 1) to store the already visited parents found in a depth-first search (DFS) path in order to check if an edge contains an already visited parent (i.e. a cycle is present or not). We have defined variables as TypeInvariant (line 5) to check for type violation. There is one operator, IsEmpty (line 6), defined to check if the graph is empty or not, and it is used in specifying other operations. The set prime (line 2) contains prime numbers from 2 to 200 for labeling the graph. The labeling scheme for the DAG was described in detail in Chapter 4. 


\subsubsection{ADAG operations}

In this section we formally specify 4 of the ADAG operations, includes, excludes, depend and descendants. We also calculate the compute time for depend and descendants.

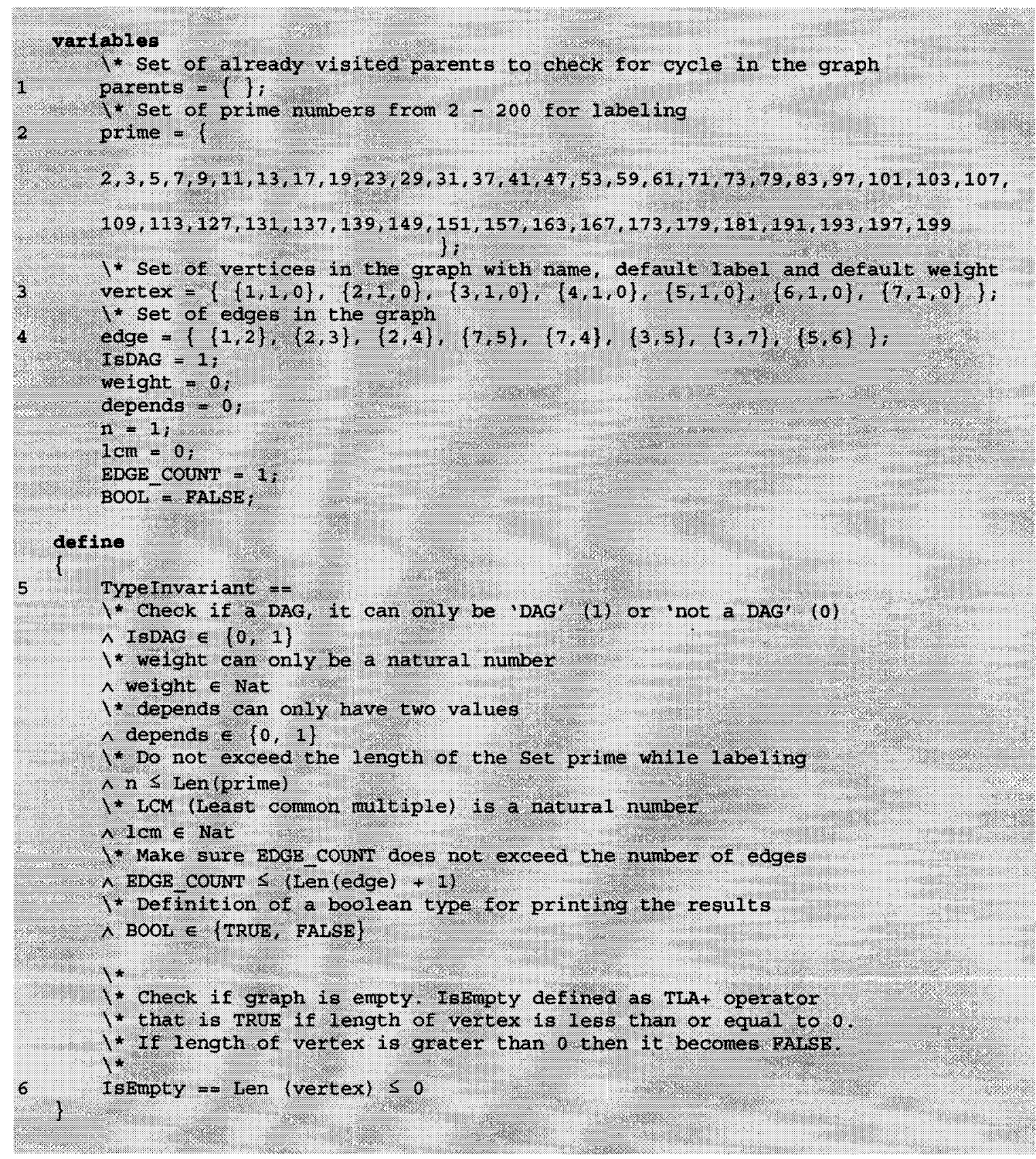

Figure 5.1 Global variables (data) and the define block 


\subsubsection{Includes}

Figure 5.2 specifies the operation includes. It has 1 input parameter, the vertex $i v$. It utilizes the user-defined TLA+ operator IsEmpty defined in Figure 5.1 to check if the graph is not empty. Then it checks if the set vertex contains the input vertex $i v$.

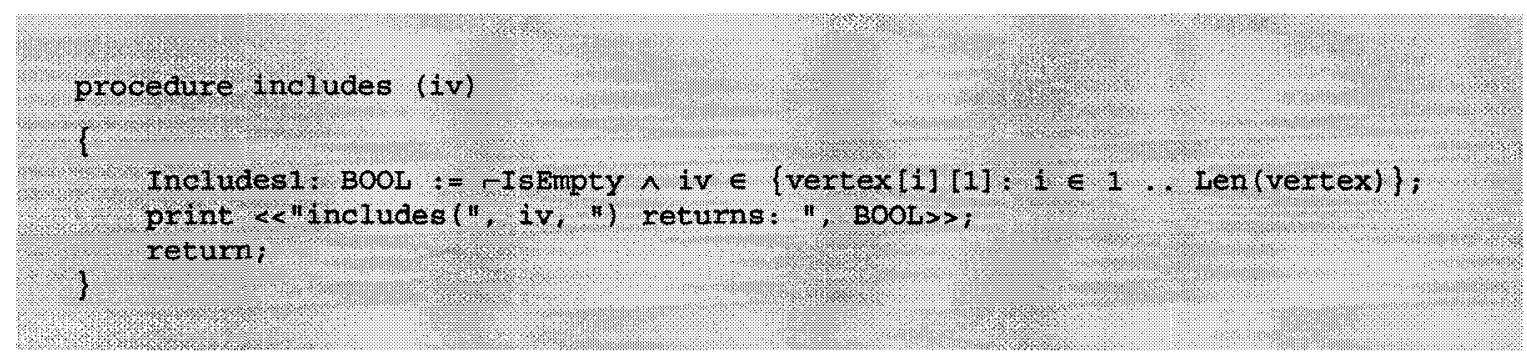

Figure 5.2 ADAG operation includes

\subsubsection{Excludes}

Figure 5.3 specifies the operation excludes. It has 1 input parameter, the vertex $e v$. It also utilizes the user-defined TLA+ operator IsEmpty to check if the graph is empty. Then it checks if the set vertex does not contain the input vertex $e v$.

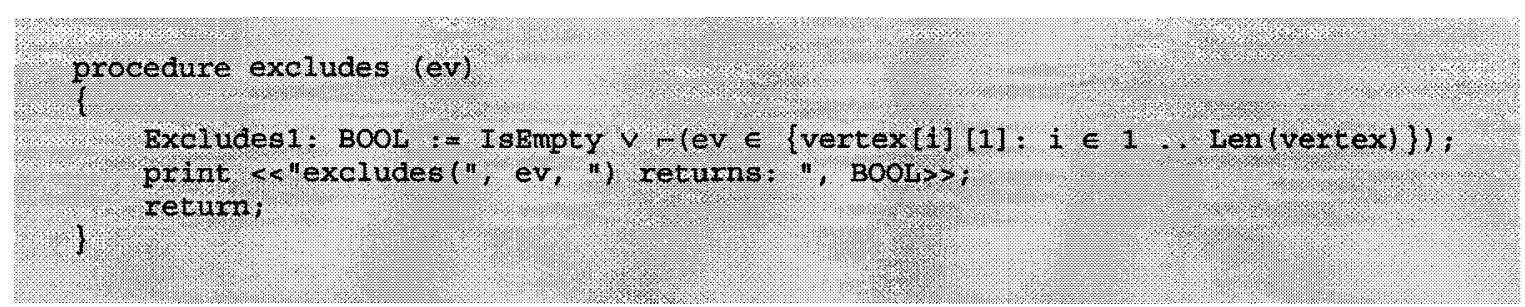

Figure 5.3 ADAG operation excludes

\subsubsection{Depend}

Figure 5.5 specifies the operation depend. It has 2 input parameters, $v 1$ and $v 2$, and checks for dependency between these two vertices. We assume that the graph is 
already labelled as described in Section 4.1. To check for dependency we only need to determine if the bigger label is divisible by the smaller label. The procedure CheckDependency is used in procedure depend for computing the mod and checking for dependency between two vertices, as shown in Figure 5.4.

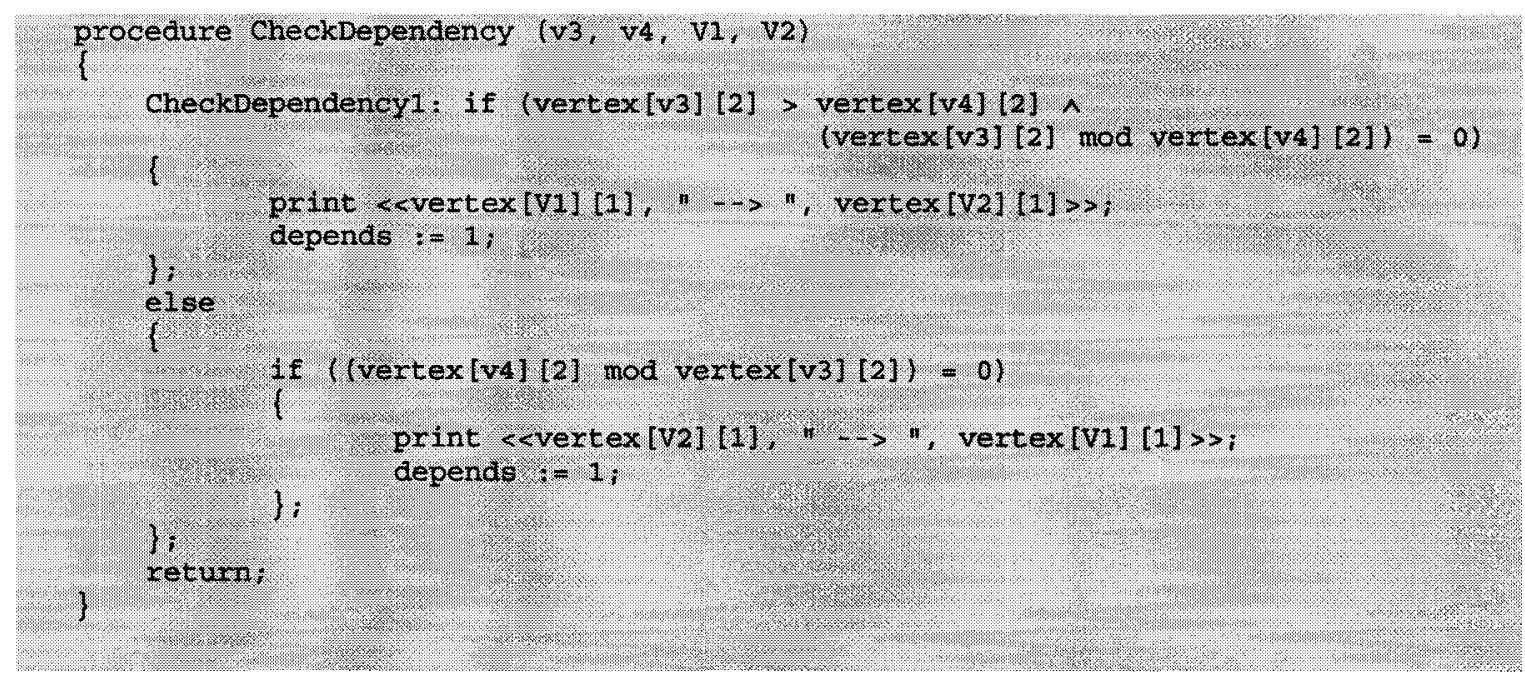

Figure 5.4 ADAG sub-operation CheckDependency

The labeling scheme described in Section 4.1 labels all the leaves of the graph as 1 , so we have 2 cases to check before checking for dependency. The first case (line 01 to line 05 ) is when both labels of vertex v1 and v2 are not 1 . In this case we simply call CheckDependency (line 02) and there is no loop involved, so the compute time is $O(1)$. The other case (line 06 to line 26) is when the label of one of the vertices is 1 (a leaf). In this case we call CheckDependency (either line 14 or 23) for each one of the immediate parents of the vertex with label 1 . If any one of these parents is dependent on the other vertex, then the vertex (with label 1) is also dependent on the other vertex (whose label is not 1) and the procedure returns. There is only one loop (either at line 08 or 17) involved in this procedure, and the maximum loop number is the number of immediate parents of 


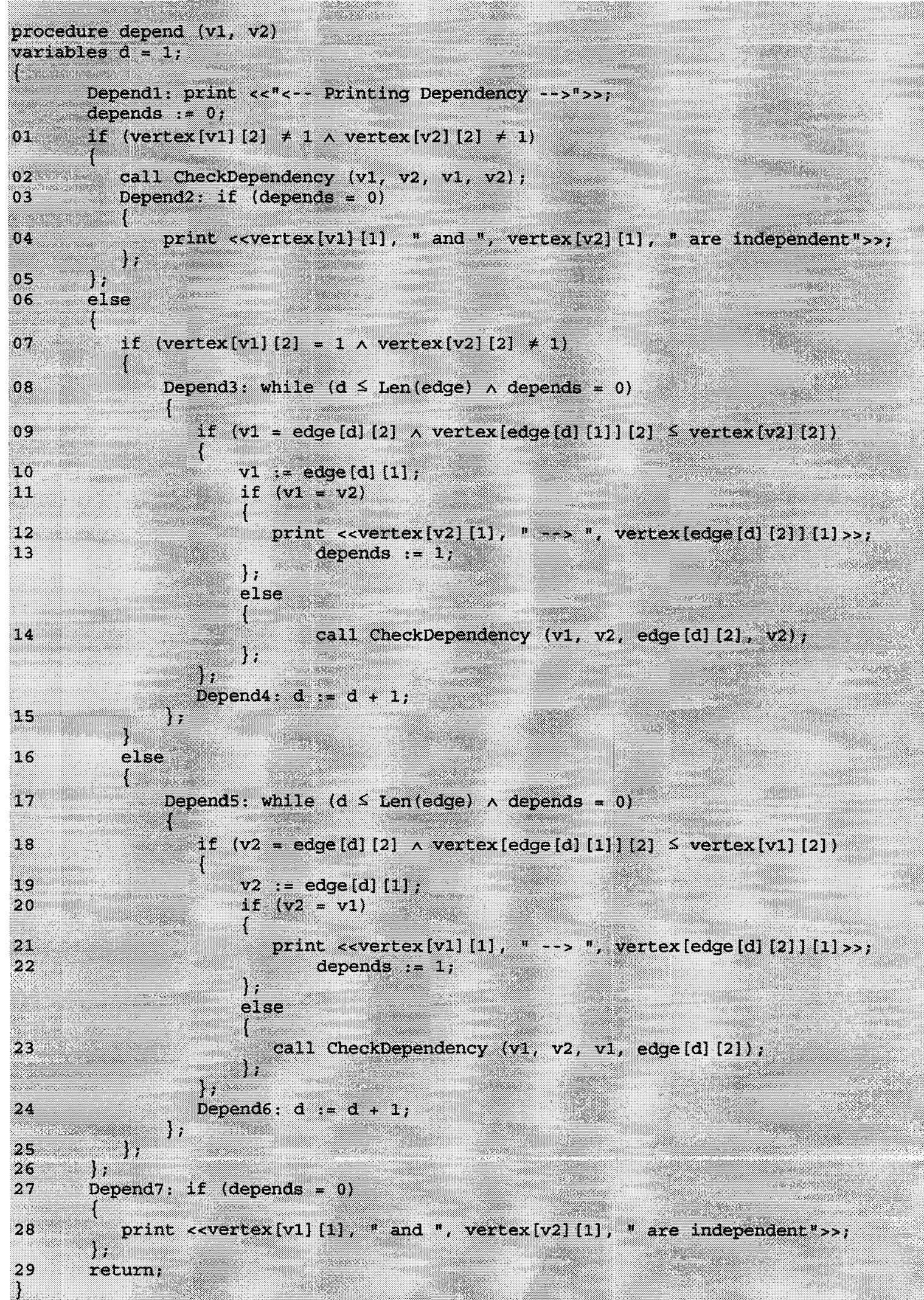

Figure 5.5 ADAG operation depend 
the vertex with label 1 , so the compute time for this case is $O(P)$, where $P$ is the number of immediate parents of the vertex with label 1 . Hence, the compute time for this operation is $O(P)$ if the label of one of the vertices is 1 and $O(1)$ otherwise.

\subsubsection{Descendants}

Figure 5.6 specifies the operation descendants. Since in this operation only one vertex (i.e. the input vertex) is compared with all other vertices, it is not optimal to call the operation depend for all the vertices and check three cases every time for the two

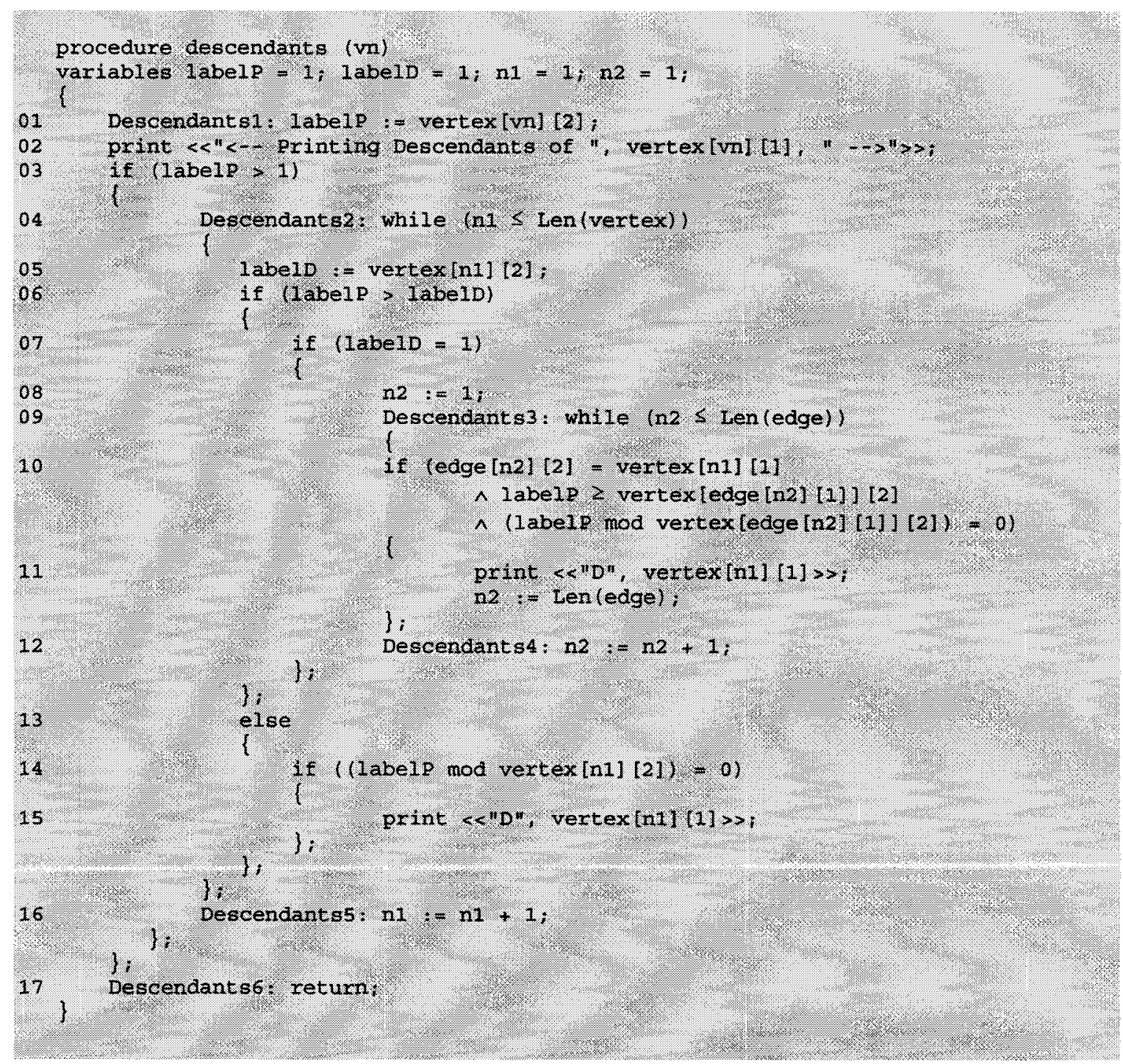

Figure 5.6 ADAG operation descendants 
vertices. Therefore, we do not use the operation depend in this operation.

In this operation there are also two cases, but different than the two cases in the operation depend: when the label of the vertex is 1 (line 03) i.e., a leaf, and vice versa (line 17). It iterates the set vertex and computes the mod to check for dependency between the input vertex, $v n$, and all other vertices.

The ADAG is stored in a simple list whose length is $n$, where $\mathrm{n}$ is the number of vertices in the ADAG. The loop to find descendants in Figure 5.6 executes for the length of the list, so the total number of operations is equal to the length of the list, which is the number of vertices in the DAG. Hence, the time complexity for this operation is $O(n)$, both in the average and worst cases, where $\mathrm{n}$ is the number of vertices in the ADAG.

\subsubsection{CAL actions}

All CAL actions were informally defined and explained in Section 3.3 using OCL-like syntax. The most important action in CAL is the creation of the ADAG, which includes ADAG labeling and setting the weight for each vertex. The ADAG labeling scheme was described in Section 4.1, so here we formally specify and explain the operation and execution of the action create and its sub-actions, relabel and setweight.

\subsubsection{Create}

Figure 5.7 specifies this action. Since we have already defined (with name, default weight and label, as shown in Figure 5.1) one of the input sets, vertex, in the action create we simply call setweight and relabel to set the weight and label of each vertex in the set vertex. The purpose of this specification is not to test the performance of CAL actions, but the operation and functionality of these actions. Therefore, this action does not add any vertex or edge (to save time, they are already defined in Figure 6 as 
variables), it emulates adding a vertex and an edge to the graph by using a variable EDGE_COUNT, which counts the current number of edges processed. It adds them one by one and increments the EDGE_COUNT with each addition, so the sub-actions setweight and relabel do not check beyond the number EDGE_COUNT. Hence, it implies that only EDGE_COUNT number of edges have been added.

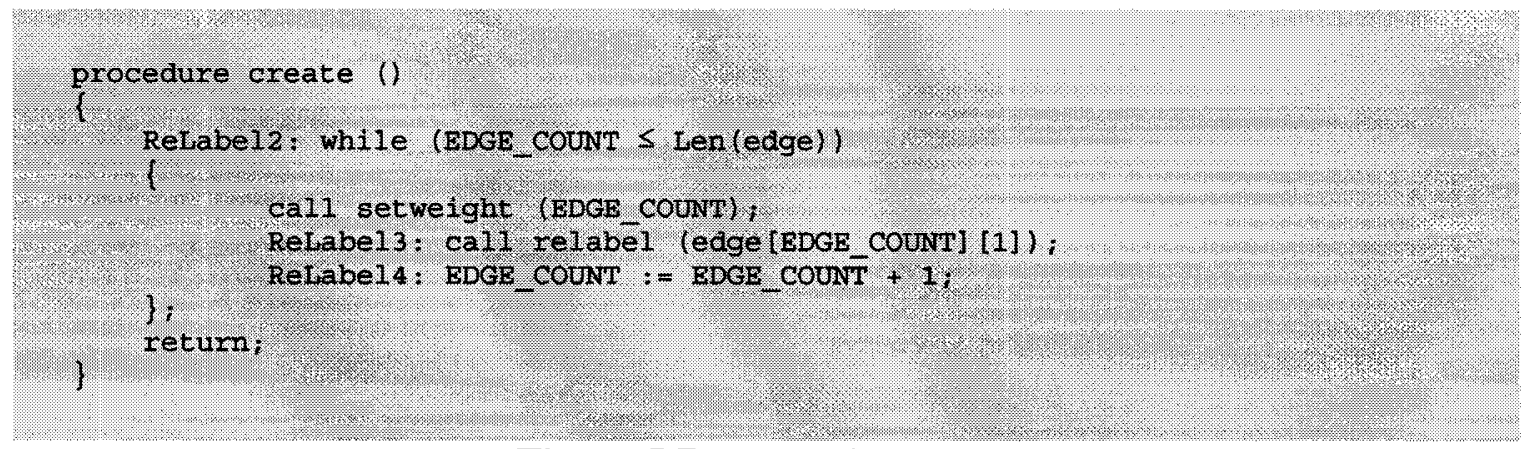

Figure 5.7 CAL action create

\subsubsection{Relabel}

Figure 5.8 specifies this operation. It has one input parameter vertex, $r p v$. This operation calls (line 1) the operation Label to label each vertex. The Label operation uses the labeling scheme (Section 4.1) to label a vertex and is formally specified in Appendix E. Whenever a vertex and an edge are added to the graph, all the ancestors of that vertex are relabelled using a top-first search (TFS) graph traversing technique, which is explained below. Since it traverses all the ancestors of a vertex, we also set (add) the weight of each parent depending on the relationship that it has with its children. This weight is set and defined in the setweight operation. In this way, we determine the weight of each vertex by adding the weights of all its descendants (as defined in Equation 3.1).

The loop in Figure 5.8 at line 2 iterates over the set edge using the count 
EDGE_COUNT to emulate adding edges as described above. The procedure relabel checks (line 3) if the input vertex $r p v$ is the child of any vertex in the set $e d g e$ (i.e. it finds all the immediate parents of the vertex rpv). If it finds any parent (line 3), then it recursively calls itself (line 4) to relabel the parent. It keeps finding till it reaches the top in a straight path. Then it backtracks to the most recent vertex that has not been explored and repeats the same steps. Since it goes to the top instead of going to the bottom, we call this method TFS.

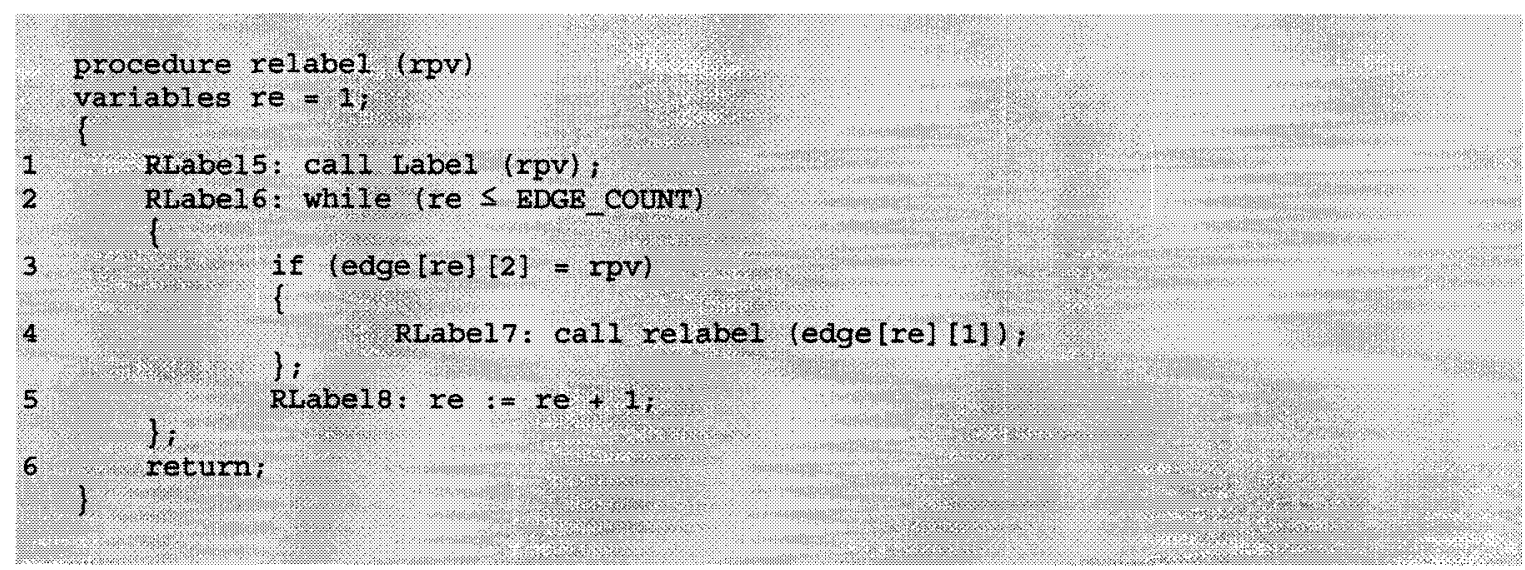

Figure 5.8 CAL sub-action relabel

\subsubsection{Setweight}

Figure 5.9 specifies this operation. It has one parameter, the edge en. This operation sets the weight of the edge en according to the dependency relationship defined in the configuration file in Appendix E. It sets the weight using the rule $R 1.5$ defined in Section 5.1.

\subsection{Formal verification using TLC}

TLC is a model checker for finding errors in TLA+ specifications. Formal specifications of the CAL model in TLA+ helped us to use TLC for verifying the CAL 


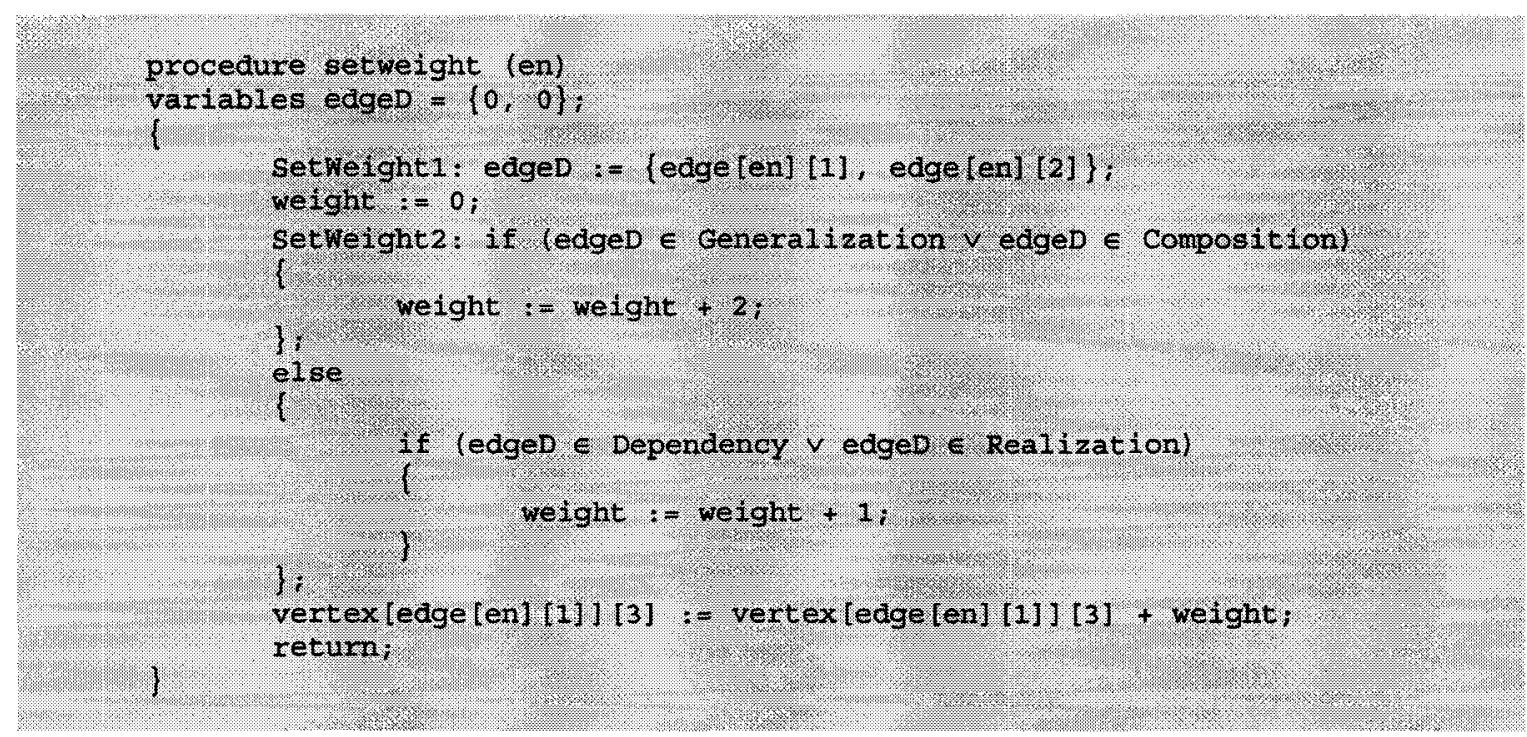

Figure 5.9 CAL sub-action setweight

model. Figure 5.10 shows a part of the main block of the CAL specifications, and Figure 5.11 shows part of the listing of TLC output. The complete CAL specifications and the TLC output appears in Appendix E. In this section, we describe the verification of the ADAG operations and CAL action and sub-actions specified in Section 5.2.2. The data used is listed in Figure 5.1.

First, TLC compiles all the files declared (included) in the TLA+ specification file and then the TLA+ file itself. In this thesis, the TLA+ specification file for CAL is CALM.tla (a full listing of this file is given in Appendix E). The TLC checks the specifications defined in the configuration file (CALM.cfg appears at the end of Appendix E). The specification to be checked is defined as Spec (temporal formula) in CALM.tla as follows:

1* In TLA+, the symbol $\triangleq$ means is defined equal to and the $\backslash$ * temporal formula $\square F$ asserts that $F$ is always TRUE.

$1^{*}$ Hence, this formula asserts that the initial state satisfies Init, $I^{*}$ and every other state satisfies Next. All the procedures called 
$1 *$ in Main block, shown in Figure 5.10, becomes the Next state.

Spec $\triangleq I$ Init $\wedge \square[\text { Next }]_{\text {vars }}$

The language used for the specifications in the file CALM.tla is pluscal, which is based on TLA+, so these specifications are translated to TLA+ by the TLC before compiling. Every procedure in the main block in the file CALM.tla is translated and added to the next state. TLC first checks the initial state (defined as Init in CALM.tla)

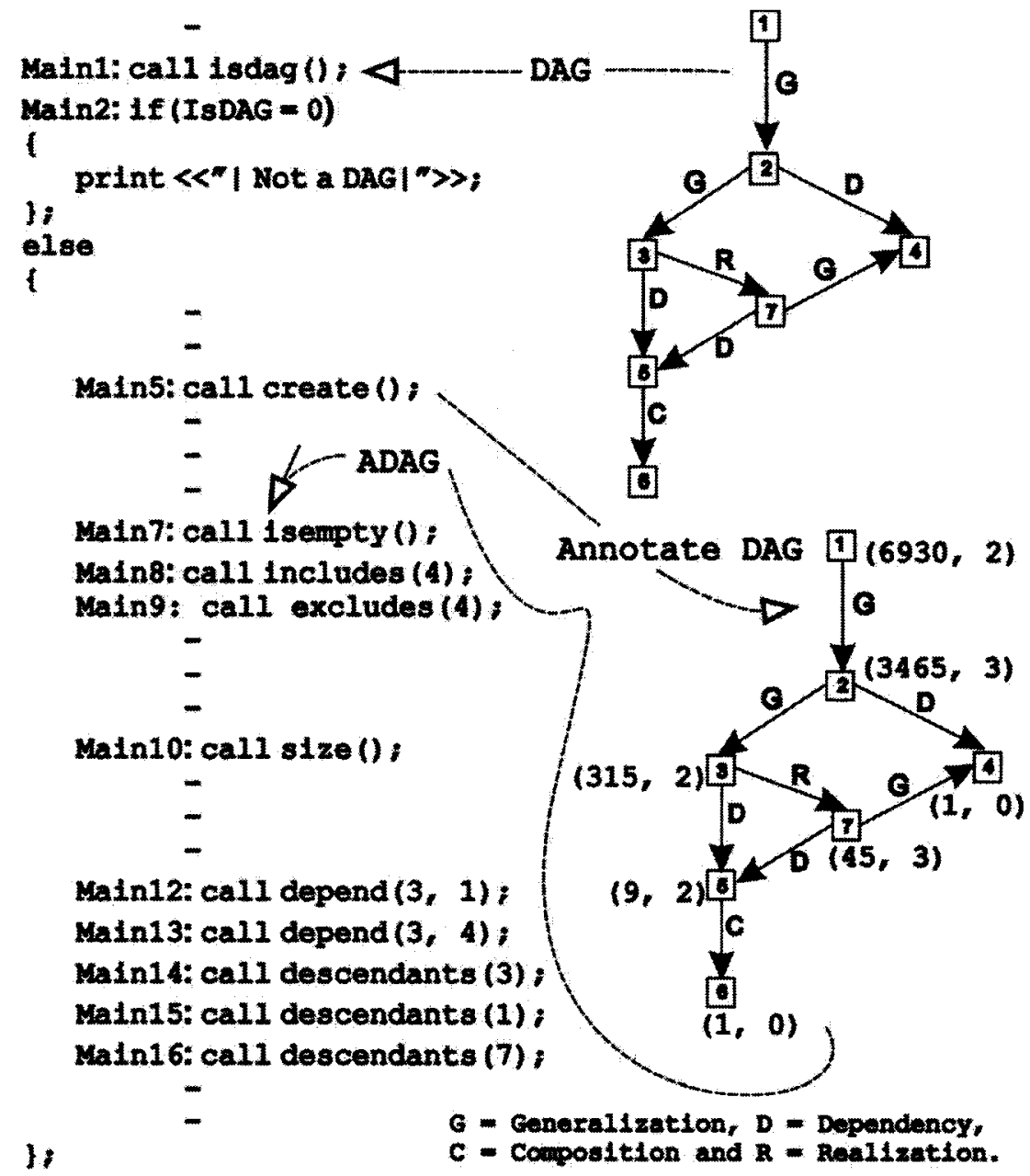

Figure 5.10 Part of the main block of the CAL specifications, and the CAL data structure (ADAG) before and after annotations 
TLC Version 2.0 of January 16, 2006

Model-checking

Parsing file CALM.tla

Parsing file C: \TLA \tlasany \StandardModules\Naturals.tla

Parsing file $c: \backslash T L A \backslash t$ lasany $\backslash$ StandardModules $\backslash$ Sequences.tla

Parsing file C: \TLA $\backslash$ tlasany \StandardModules $\backslash T L C . t l a$

Semantic processing of module Naturals

Semantic processing of module Sequences

Semantic processing of module TLC

Semantic processing of module CALM

Finished computing initial states: 1 distinct state generated.

$\ll " \mid$ Start Test |" >>

$\ll$ "<-- Creating ADAG (Labeling and setting Weight) -->" >>

$\ll$ "Vertex, label, weight $-\rightarrow ", 1,2,2>$

$<$ "Vertex, label, weight $->1,2,3,2>>$

$\ll$ "Vertex, label, weight $-\rightarrow ", 1,6,2$ >

$\ll$ "Vertex, label, weight $-->", 2,3,3>>$

$\ll$ "Vertex, label, weight $-\rightarrow ", 1,6,2 \gg$

$\ll$ "Vertex, label, weight $->", 7,5,1>>$

$<$ "Vertex, label, weight --> ", $7,5,3$ >>

$<$ "Vertex, label, weight $\rightarrow$ " $3,7,1 \gg$

$\ll$ "Vertex, label, weight $-.>1,2,21,3>$

$\ll$ "Vertex, label, weight $-.>", 1,42,2 \gg$

$\ll$ "Vertex, label, weight $->1 ", 3,35,2>>$

$\ll$ "Vertex, label, weight --> ", 2, 105, $3 \gg$

$\ll$ "Vertex, label, weight $-\rightarrow ", 1,210,2$ >>

$<$ "Vertex, label, weight $-->1,5,9,2>>$

$\ll$ "Vertex, label, weight $->1,7,45,3>$

$\ll$ "Vertex, label, weight --> ", 3, 315, 2 >>

< "Vertex, label, weight --> ", 2, 3465, 3 >

< "Vertex, label, weight --> ", 1, 6930, 2 >>

$\ll$ "Vertex, label, weight $-.>n, 3,315,2>$

$\ll$ "Vertex, label, weight --> ", 2, 3465, 3 >

< "Vertex, label, weight --> ", 1, 6930, 2 >

《 "isempty() returns: ", FALSE >

$\ll$ "includes(", 4,") returns: ", TRUE >>

$<$ "excludes(", 4, ") returns: ", FALSE >

< "SIZE: ", 7 >

$<<"<-$ - Printing Dependency -->" >>

$\ll 1, "-->", 3>$

$<$ "<-- Printing Dependency -->" >>

$\ll 3, "-->\|, 4>$

$<$ «<-- Printing Descendants of ", 3, " -->" >>

$<$ "D", $4 \gg$

$<$ "D", $5>$

$<$ "D", $6>>$

$\ll$ "D", $7>$

$<1<--$ Printing Descendants of ", 1, " - ->" >>

$<$ "D", $2>$

$<$ "D", $3>$

$\ll " D ", 4>>$

$<$ "D", $4 \gg>$

$\ll$ "D", $5 \gg$

$<$ "D", $6>$

$<$ "D", $7>$

$<<"<--$ Printing Descendants of $", 7, n-->n>>$

$<<" D ", 4>>$

$\ll$ "D", $5 \gg$

$<$ 《D", $6>>$

$<" \mid$ End Test |" >

Model checking completed. No error has been found.

9548 states generated, 9547 distinct states found, 0 states left on queue.

The depth of the complete state graph search is 9547 .

Figure 5.11 Part of the TLC output 
and the next state (defined as Next in CALM.tla). In the initial state TLC initializes all the global variables defined in Figure 5.1. Then TLC starts generating and executing all the states defined in the next state. While executing these states, TLC checks all the type invariants (defined as TypeInvariant in Figure 5.1) and reports an error if any one of these types is violated. TLC also checks and reports any deadlock in the states. 


\section{Chapter 6}

\section{CAL Prototypes}

In this chapter we discuss two prototypes implemented in this thesis: the Constraints with Action Language (CAL) parser in detail and the tool visual CAL (VCAL) in brief. VCAL loads a UML model (as XMI) and uses the CAL parser to parse CAL files. A CAL interpreter has been implemented in Java to be used in VCAL. VCAL interprets the CAL actions and operations in a CAL file and then executes these actions and operations on the CAL data structure (ADAG). The CAL parser's UML Class Diagram, shown in Appendix D, is used in this chapter as a case study to explain and test VCAL. The test and the results obtained after running VCAL are described in Section 6.4, and the same are available as an online demonstration [63] prepared for this thesis.

\subsection{CAL parser}

This section gives a detailed overview of the implementation of the CAL parser. All the major classes are described, including the data structure used for storing the symbol table and the abstract syntax tree (AST). An XML file is shown for describing the output AST.

Parsing is the process of analyzing the input sequence (a string) in order to determine its structure according to a formal grammar. Parsers usually operate in two stages. The first stage is the lexical analysis in which the input is scanned and tokens are generated. The second stage is syntax analysis in which the input and tokens are 
converted to a parse tree. For efficiency, these two stages are performed in parallel in the CAL parser.

\subsection{Implementation details}

A predictive parser [3] has been implemented manually in $\mathrm{C}++$ to validate the grammar and generate the syntax tree. The rationale for using this technique is as follows: The comparison in Table 6.1 tells us that if the grammar can be transformed to fit LL(k) then LL parsing should be used, and the CAL parser implemented for this thesis shows that an LL(1) parser can be implemented manually even if the grammar is not LL(1). Although the grammar was optimized for LL parsing by removing left recursion, to keep the size of the grammar manageable left factoring was not applied to the grammar. Rather, left factoring was implemented in the CAL parser for optimization. If the grammar is more complex, then automatic parser generators should be used for LR parsing.

The CAL parser is compiled for the Microsoft Windows platform under Cygwin, but it is easily ported to other platforms (e.g. UNIX, Linux etc.). The grammar and the parser have been optimized for LL(1) parsing using techniques mentioned in Chapter 2. The state machine for lexical analysis and the syntax analysis tree are shown in Appendix B and Appendix C respectively. All the tokens for the parser are listed in Appendix A. To improve the speed of scanning, which is the most time consuming process in parsing, a CAL file is read and stored in memory as a single buffer. The parser is designed such that keeping just one token in its memory it builds the syntax tree as it scans the file buffer. Because of the length of the source code of the parser, it is not included in this thesis. A soft copy of the source code is also available online [64]. 
Table 6.1 Comparison of LL and LR parsing

\begin{tabular}{|l|l|}
\hline \multicolumn{1}{|c|}{ LL parsing } & \multicolumn{1}{c|}{ LR parsing } \\
\hline Simple & Complex \\
\hline$*$ Less number of grammars supported & More number of grammars supported \\
\hline$* *$ Less space (table size) & $* *$ More space (table size) \\
\hline Better parsing speed & $\begin{array}{l}\text { Comparable parsing speed with } \\
\text { optimization }\end{array}$ \\
\hline $\begin{array}{l}\text { Actions can be placed anywhere } \\
\text { without conflict }\end{array}$ & $\begin{array}{l}\text { Actions cannot be placed anywhere } \\
\text { without conflict }\end{array}$ \\
\hline Better error repair and reporting & Normal error repair and reporting \\
\hline
\end{tabular}

* Because of left factoring and left recursion

** If table driven parsers

The UML Class Diagram for the CAL parser is shown in Appendix D, and detailed documentation of the complete API (i.e. all 15 CAL parser classes) is available online [57]. Following is a description of the main features of each major class in the CAL parser.

\subsubsection{Class Parser}

Class Parser reads the token and analyses them according to the grammar, from left to right. For example, the first token it reads is either a comment or a package. After it reads the token package, it scans the context statements, which are of three types, attrOrAssocContext, classifierContext and operationContext. A separate procedure is implemented in this class for each non-terminal in the grammar. 


\subsubsection{Class OclStmt}

Class OclStmt contains $1400+$ lines of $\mathrm{C}++$ source code and is the biggest class of the CAL parser. It implements the methods for checking the correctness of the OclStmt and also builds the AST. The OclStmt contains most of the CAL statements, as defined in the grammar given in Appendix A. The original concrete syntax of OclStmt as described in [2] contains several ambiguous production rules. These production rules have been transformed using techniques described in Chapter 2, and new rules have also been added to remove these ambiguities.

\subsubsection{Class Lexer}

Class Lexer performs lexical analysis of a CAL file. It reads the file in a buffer, scans it token by token and passes it to the Class Parser for syntax analysis. It processes the stored buffer and looks several characters ahead of the current input position for a pattern before a match can be found. The tokens for the CAL parser are selected such that the parsing of the file becomes easier. The main procedure, getNextToken(), is implemented as a finite state machine, which is given in Appendix C.

\subsubsection{Class AST}

Class AST contains procedures for building an AST. The AST starts with a root node, and children nodes are added as a linked list to the parent node, during syntax analysis of a CAL file, by the class Parser. Figure 6.1 shows the data structure used for storing the AST and is explained in the next Section.

\subsubsection{Data structure for storing the AST}

The AST is stored as a tree. Each node is labeled as a string of numbers, which increases with the number of children as shown in Figure 6.1. The label or the $I D$ of a 
node is stored as class IntList. This is the linked list for storing integers as a list. The IntList class makes it easier for a parent node to have more than 9 children. Figure 6.2 displays the output XML file generated by the class $A S T$ from a sample CAL file. The

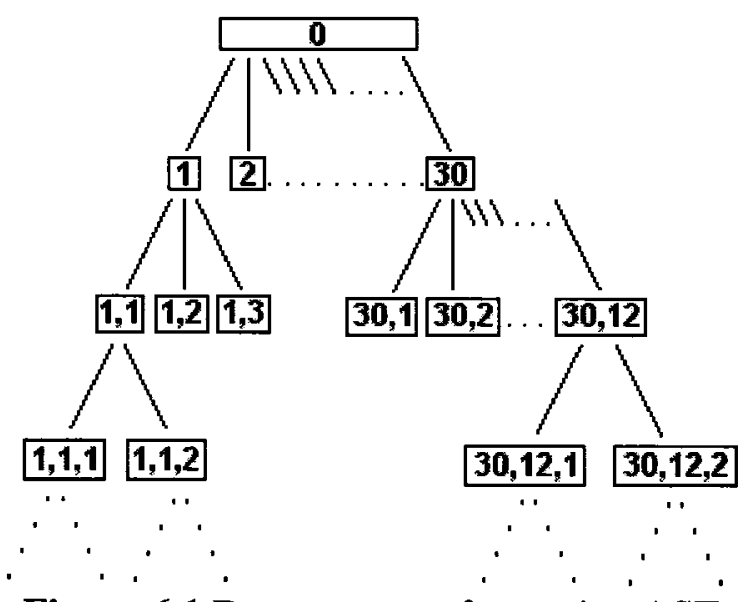

Figure 6.1 Data structure for storing AST

XML tree follows the CAL grammar syntax for easy interpretation. The tags used for the XML are the tokens, which are stored in the symbol table of the CAL parser, and the attribute, name, is the value of the token, which is also called lexeme, and is scanned by the class Lexer. The CAL file used for the example in Figure 6.2 is below:

-- Sample file created on Sep 07, 2006 for testing CAL Parser

package simple::simpleone::simpletwo

context NamedEntities

inv nameIsMandatory:

self.name.isDefined and self.name $<>$ Dude

context Person

inv: 


$$
\text { if self.sex }=\text { male then true else false endif }
$$

endpackage

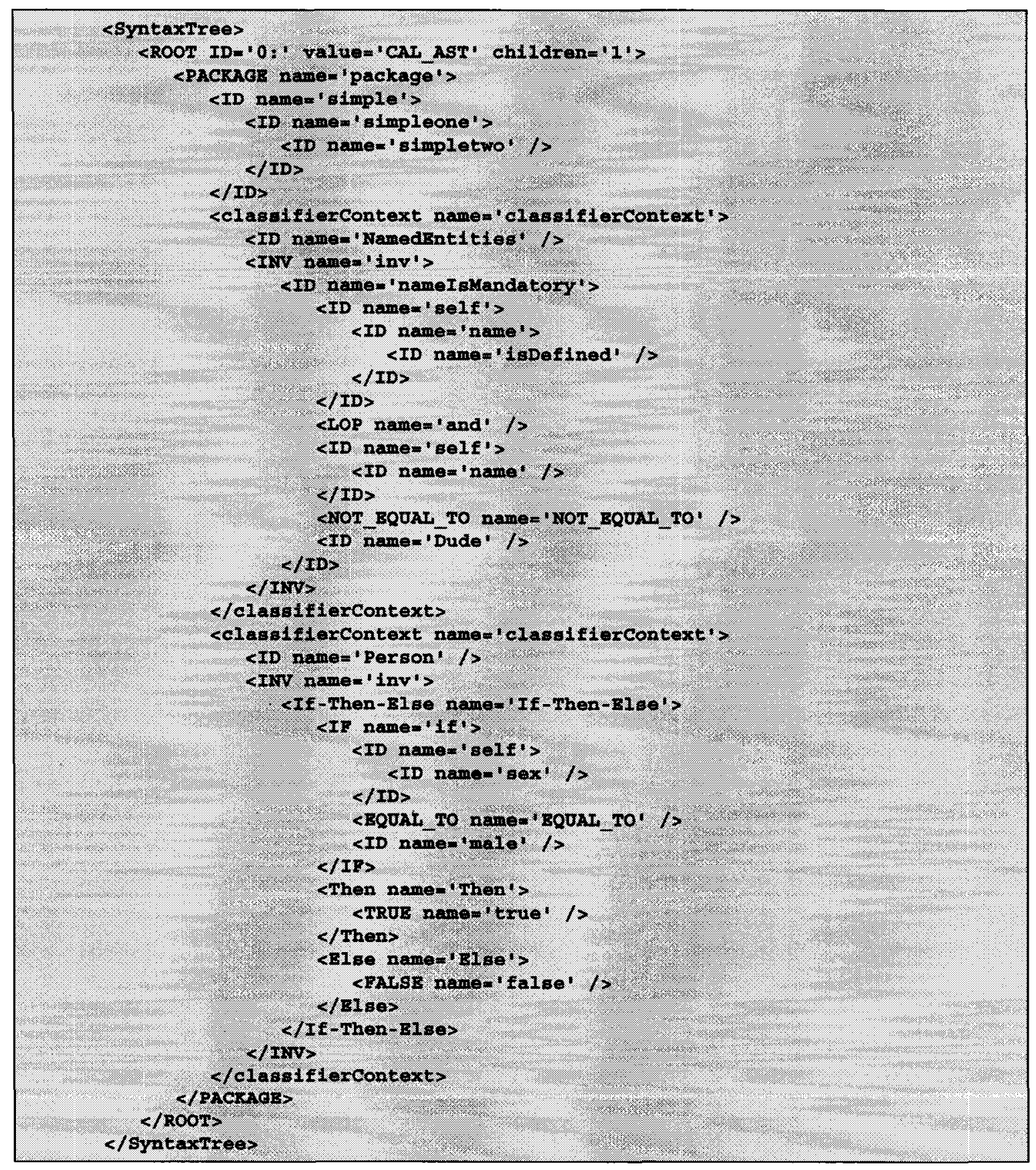

Figure 6.2 XML output generated by the Class AST 


\subsubsection{Class Symtable}

Class symtable, which is implemented as a hash table, builds the symbol table for the CAL parser and is used for storing lexemes and tokens of the language. A symbol table is built during lexical analysis and is used during syntax analysis of a CAL file. Three procedures, lookup, insert and remove have been implemented for this class to manipulate the symbol table. The popular $P J$ Weinberger $C$ compiler hash function (PJW) as described in [3] has been used for storing tokens in the symbol table. There is a chance for collision between the keys generated by any hash function. The following section describes how this collision is avoided in the implementation of the symbol table.

\subsubsection{Collision resolution}

If two keys have the same index, the corresponding value cannot be stored in the same location in the hash table. If the location is already occupied in the hash table, then we need to find another location to store this value and do it in such a way that while looking up we can find it. There are a number of collision resolution techniques, but here we describe two of the most popular ones. Table 6.2 compares these two techniques.

\subsection{Chaining}

This technique uses the concept of buckets in the form of a linked list to store keys in the hash table. Each slot in the hash table is a reference to the linked list. When a key collides (i.e. has the same value as the other key) with another key, then the data associated with that key is inserted in the same slot to the linked list. The data can be inserted at either end of the linked list and can be searched and removed using the list.

As shown in Figure 6.3, a linear list is used to store keys in the vertical direction, and buckets are used to store key values in a horizontal direction. Edlam C and Tgam B 
are the two keys that give the same hash index if we use the PJW hash function, so they are stored in the linked list at the same index (hash value), which is 24 . To lookup the key Edlam $C$ first we go directly to index (hash value) 24 in the array and then search the linked list for the same key, which in this case is the first element of the linked list. This same technique is used in implementing the symbol table for the CAL parser.

\subsection{Open addressing}

This technique stores the key value directly in the hash table. Collision is resolved by searching through alternate locations in the array for the key. The key is inserted by probing for an unused slot in the hash table. To lookup a key, first the target key is compared at the index (hash value). If it is not found, then probing is used to find the next index and so on until the key is found. Figure 6.4 shows the same hash table as shown in Figure 6.3, but the keys are inserted differently. The key Edlam $C$ is inserted at index (hash value) 24 and key Tgam $B$ is inserted at index (hash value) 25, which is the next unused slot in the hash table.

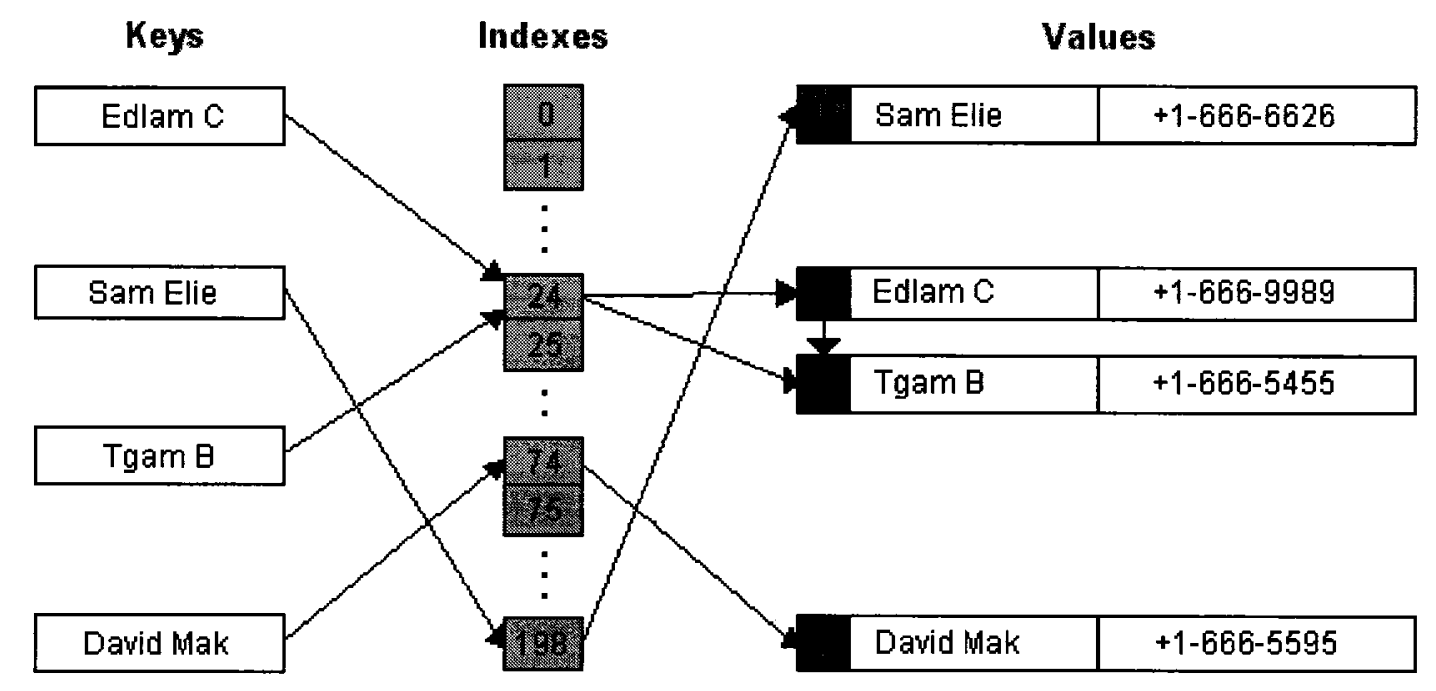

Figure 6.3 Collision resolution by chaining 


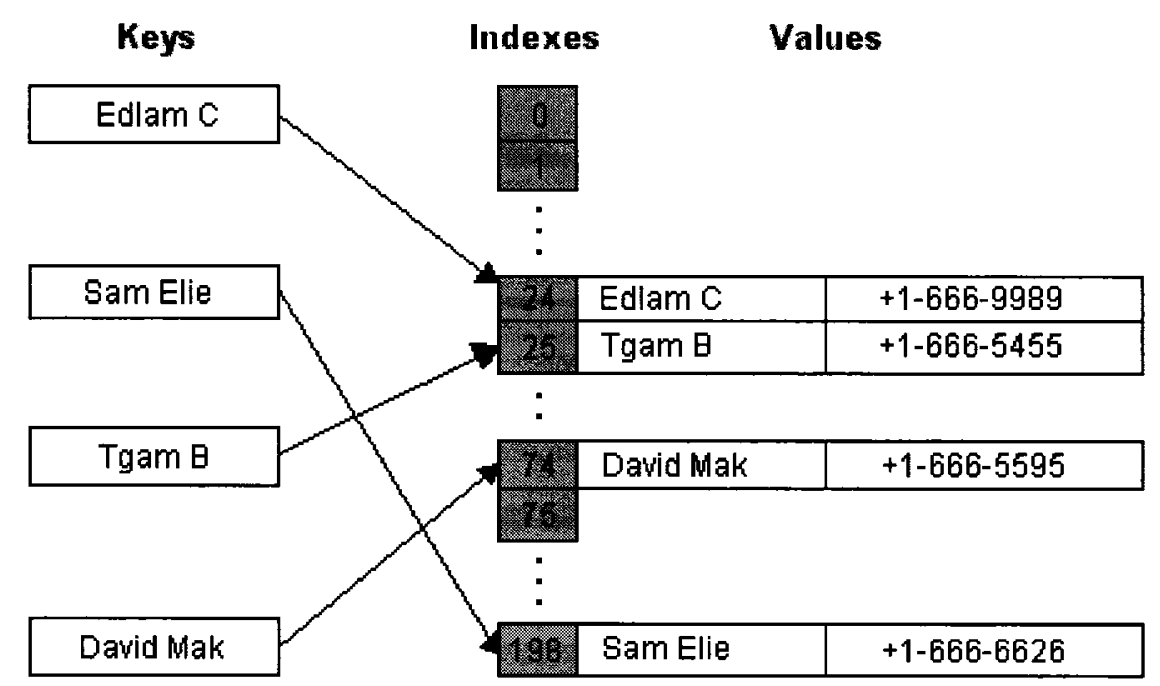

Figure 6.4 Collision resolution by open addressing

Table 6.2 Chaining versus open addressing

\begin{tabular}{|l|l|}
\hline & \\
\hline Simple to implement & Difficult to implement \\
\hline Insensitive to clustering & $\begin{array}{l}\text { Require better hash function to avoid } \\
\text { clustering }\end{array}$ \\
\hline $\begin{array}{l}\text { Table cannot fill up and doesn't show } \\
\text { sudden increase in lookup time }\end{array}$ & $\begin{array}{l}\text { Sudden increase in lookup time in near-full } \\
\text { table }\end{array}$ \\
\hline $\begin{array}{l}\text { Use less space if table is sparse (more } \\
\text { free slots) }\end{array}$ & $\begin{array}{l}\text { Use more space if table is sparse (more } \\
\text { free slots) }\end{array}$ \\
\hline Use less space if the value is larger & Use less space if the value is small \\
\hline Memory allocation overhead & No Memory allocation overhead \\
\hline Difficult to serialize & Easier to serialize \\
\hline
\end{tabular}

Different probing techniques are used in open addressing hash tables. The basic difference between these techniques is the interval used for probing. Some of them are 
Linear probing in which the interval between probes is fixed at 1, Quadratic probing in which the interval between probes increases linearly and is defined by a quadratic function, and Double hashing in which the interval between probes is fixed for each value. This fixed value is computed by using another hash function. The other open addressing methods are not listed here but can be found in [8].

\title{
6.2.6 Class LG
}

Class $L G$ stores all the tokens defined in the CAL grammar. It also contains a method that converts and returns the token as a string for debugging and printing purposes.

\subsubsection{Class List}

Class List implements a linked list, which is used by different classes as their data structure. Class $A S T$ uses this class to store the list of integers. The list of integers is used to label each node in AST as described in Section 6.2.4. Class Symtable uses this class to implement buckets for collision resolution as described in Section 6.2.5. The Linked list as implemented in this thesis is shown in Figure 6.5. An iterator has also been implemented for the class List. Following is a small part of the code that deletes data stored at all the nodes from the list using iteration. First it initializes the iterator and then iterates in a loop to delete the data. The iterate() method increments the iterator and returns a pointer to the data.

\author{
list.initIterator(); \\ while $(($ nodeData $=$ list.iterate ()$) !=N U L L)$ \\ delete (nodeData);
}




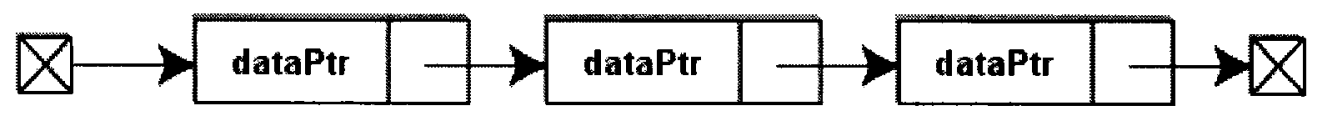

Figure 6.5 Linked list with pointer to the data

\subsection{VCAL (visual CAL)}

A visual CAL parser tool, VCAL, has been implemented in Java with a graphical user interface (GUI) using the Software Widget Toolkit (SWT) [54]. An overview of VCAL is shown in Figure 6.6. We used BigInteger API of Java 1.5 to implement the

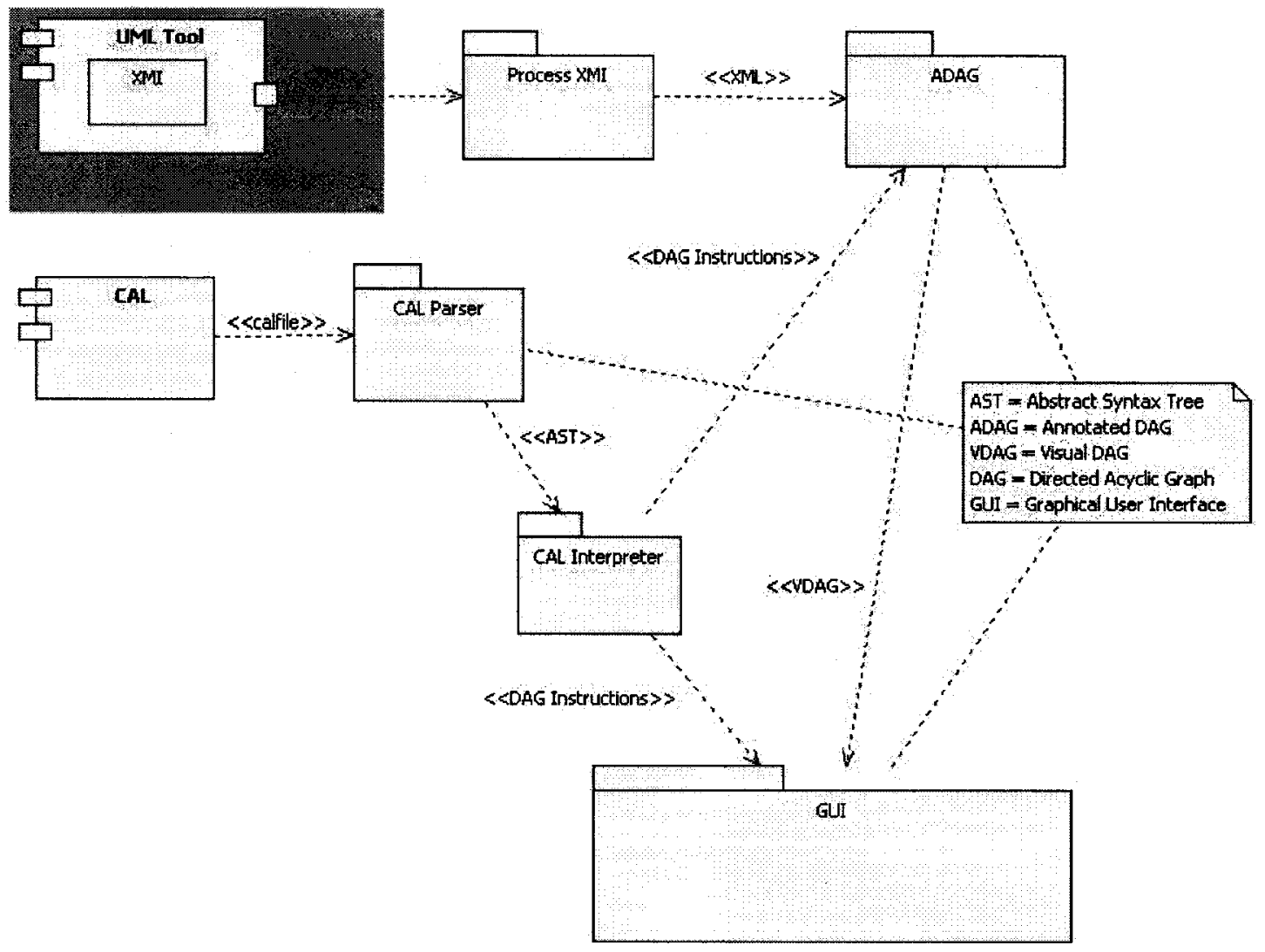

Figure 6.6 Overview of the tool (VCAL) 
ADAG labeling, so JRE 1.5 is required to run VCAL. The main components of VCAL are the CAL file, CAL Parser, ProcessXMI, ADAG, CAL Interpreter and GUI. The complete VCAL implementation consists of $15 \mathrm{C}++$ classes (discussed in Section 6.2 and listed in Appendix D) for the CAL parser and 26 Java Classes for VCAL. The complete VCAL API documentation and user manual can be found in $[58,59]$. In this section we briefly describe these components.

\subsubsection{CAL parser and CAL file}

VCAL uses the CAL parser to parse a CAL file and generate an AST in XML. The binaries of the CAL Parser are compiled for Microsoft Windows using cygwin. Cygwin is a collection of free software tools originally developed by Cygnus Solutions to allow various versions of Microsoft Windows to act somewhat like a Unix system. A cygwin.dll is included with VCAL, which is required to run the CAL parser on Microsoft Windows. The CAL parser should be able to run on other platforms (e.g. Linux, BSD and Unix), but we have not tested it on these platforms. The CAL parser is discussed in detail in Sections 6.1 and 6.2. A sample CAL file is shown in Figure 3.2 and discussed in Section 3.4 in Chapter 3.

\subsubsection{ProcessXMI}

This component loads a UML model (in XMI) into VCAL. This UML model is then processed and displayed inside the VCAL GUI. The ProcessXMI also queries the UML model and searches for all the classes in the Class Diagram. Then it uses pattern matching techniques, which use the XML document object model (DOM) and a Java XML parser, to map these classes with the dependency relationships (Section 5.1). After

processing this information, this component passes this information as an XML file to the 
$A D A G$ component for generating the annotated (with weight and label added for each vertex) DAG using the rules $R 1.1$ through $R$ 1.4, specified in Section 5.1. This DAG becomes the CAL data structure (ADAG).

\subsubsection{ADAG (annotated DAG)}

This component contains the CAL data structure (ADAG). It uses the labeling scheme discussed in Chapter 4 for labeling the ADAG. It also sets the weight of each vertex in the ADAG using rule $R$ 1.5, specified in Chapter 4. It implements all the CAL actions and operations, as defined in Chapter 3, to manipulate the CAL data structure by the CAL Interpreter.

\subsubsection{CAL Interpreter}

This component interprets CAL actions and operations. As defined in the CAL grammar CAL has three contexts. CAL actions can only be defined in an operation context, so the CAL Interpreter only interprets an operation context in the CAL AST. The interpreter reads the AST (as XML) from the CAL parser and interprets CAL actions and operations defined in the AST. A handler class is implemented to execute (handle) these actions and operations on the CAL data structure. The interpreter also updates the ADAG, which is displayed and can be interacted inside the VCAL GUI.

\subsubsection{GUI (graphical user interface)}

This component is shown in Figure 6.7. The VCAL GUI is implemented using SWT. The ADAG is displayed for visualization using an open source Java API JGraph [48], which is based on the Java Abstract Window Toolkit (AWT) [56]. JGraph is a Java graph component for the display and layout of graphs. The VCAL GUI has 5 main menus (File, Compiler, View, Vdag and Help). These 5 menus have different submenus. There 
are 14 toolbar items in the GUI. Some of these Toolbar items (Save DAG, Add, Delete, View Descendants, View Ancestors, View Both and View All) are specifically used for interacting with the ADAG. We call these toolbar items the $A D A G$ view filters, and they can be applied on any vertex in the ADAG. These $A D A G$ view filters become active only when the CAL operation view is used in a CAL file. The use of all these ADAG view filters is explained in the user manual of VCAL [59]. The toolbar items New, Open, Save, Print and Run are used as CAL file controls. For example, Run is used to run the CAL compiler for the currently opened CAL file. All the menus and toolbar items are explained in the VCAL help file.

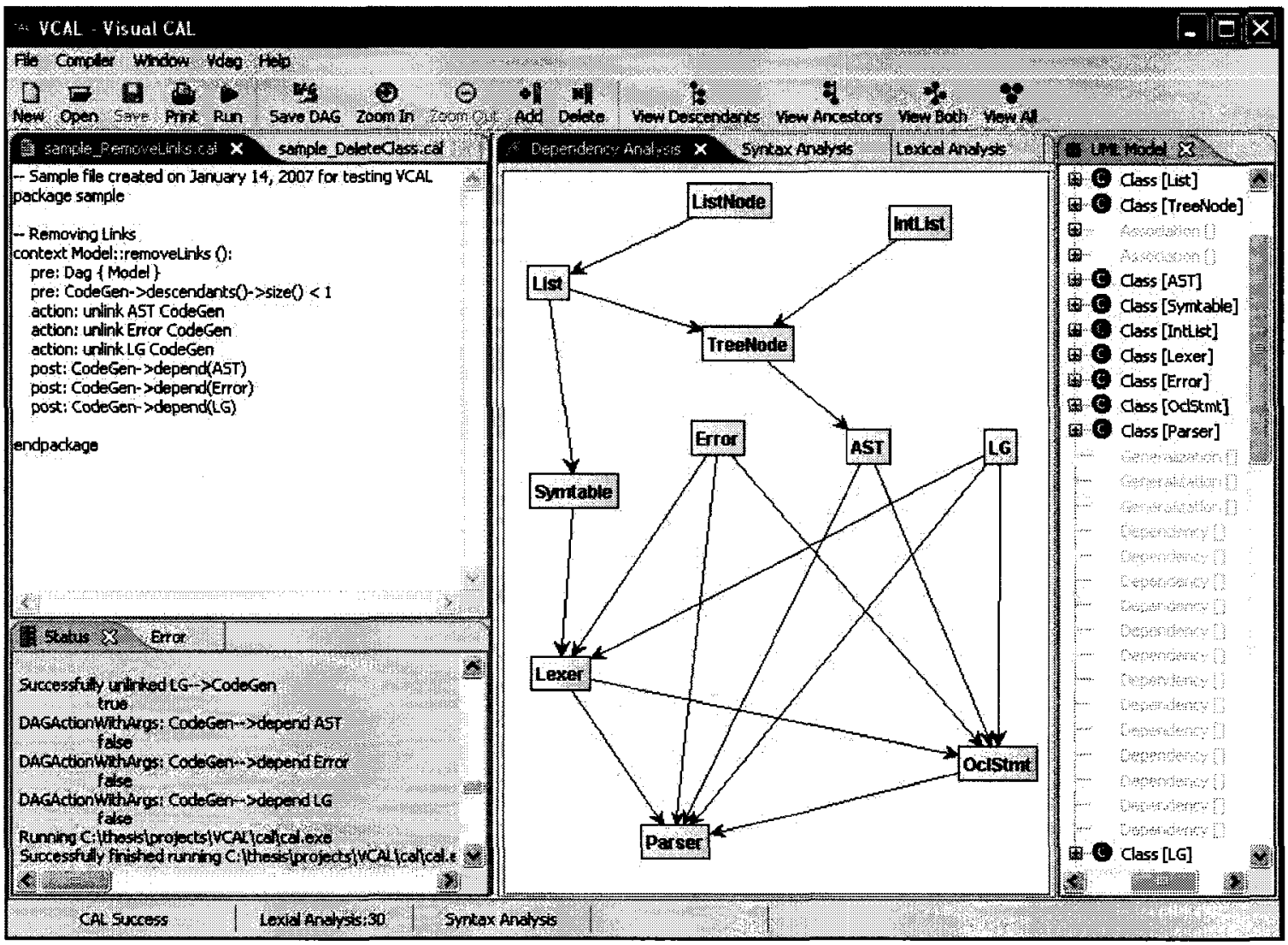

Figure 6.7 VCAL GUI 


\subsubsection{Running VCAL}

Following is the command line to execute VCAL using Java Virtual Machine (JRE 1.5):

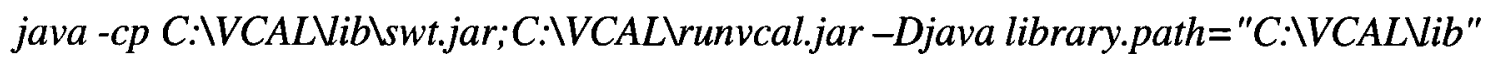
RunVCAL

where

C:\VCALVib\swt.jar and C:\VCAL\runvcal.jar are the Java jar files. The swt.jar is the jar file for SWT and runvcal.jar is the VCAL jar file. C:IVCALVib is the library path. RunVCAL is the name of the root class that calls the class VCAL that contains the static main function.

\subsection{Case study}

In this case study, we further explain the functions of VCAL and present the test results. We chose the UML Class Diagram of the CAL parser as an example for the case study. It is not the purpose to test all the functionalities of VCAL. In this case study we want to check the usage of the CAL and test the implementation of some of the CAL actions and operations mentioned in Chapter 3. Table 6.3 lists the test matrix for this case study. The rationale for choosing this example its size, i.e., its number of classes and its detailed UML Class Diagram, as shown in Appendix G for easy comprehension.

Figure 6.8 lists a part, which is relevant to this case study, of the UML Class Diagram of the CAL parser in XMI. The complete XMI file for the CAL parser has almost 1000 lines. Appendix $\mathrm{F}$ lists all the classes and dependency relationships of the UML Class Diagram of the CAL parser in more detail. The XMI file for the CAL parser is part of the VCAL package [62], as one of the sample files. There are 11 Classes, 3 generalization relationships and 13 dependency relationships, as shown in Figure 6.8. Each generalization relationship has a parent and a child, and each dependency 


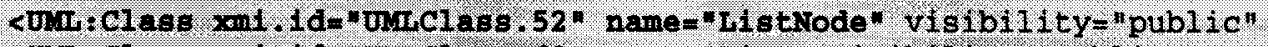

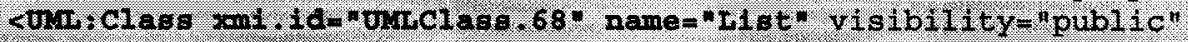

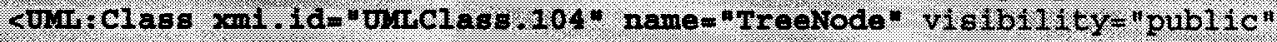

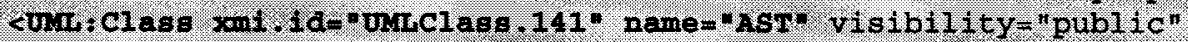

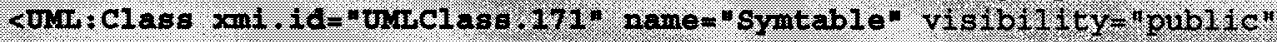

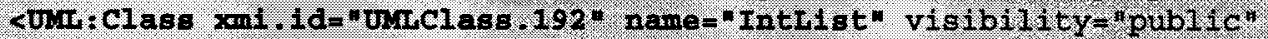

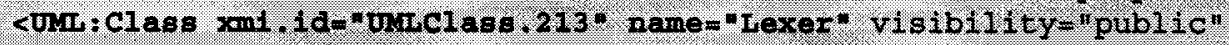

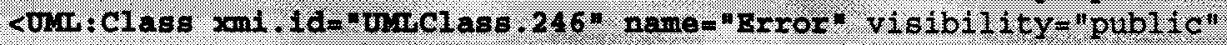

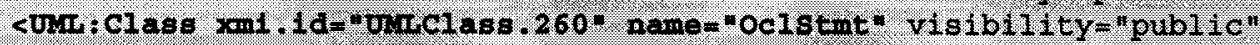

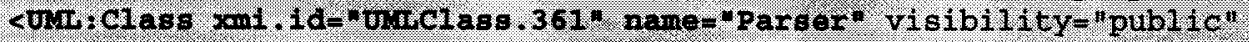

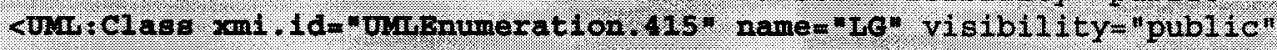

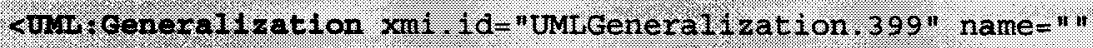

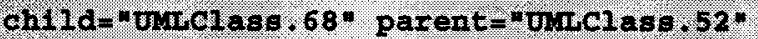

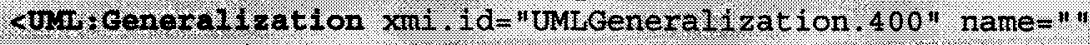

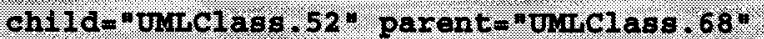

LUM. Generalization xni. 1d- "UMLGeneralization. 401 " name-" "

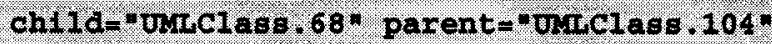

cUn: Dependency xmi. id= "umibependency. 402 " name=" $t$

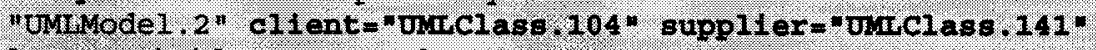
<una, Dependency xmi. Id " uMLDependency. 403 " name-" "

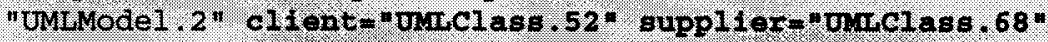
<Un: $:$ Dependency $\mathrm{xmi}$. $1 \mathrm{~d}=$ "umibependency. 404 " name=" "

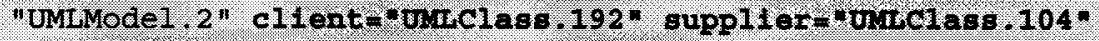
<Ua: Dependency xmi. $1 \mathrm{~d}$ - "umiDependency, 405 " name-" "

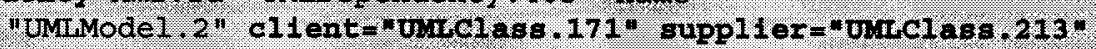
<Um: Dependency xmi. $1 \mathrm{~d}$ =" "UMiDependency, 406 " name- " "

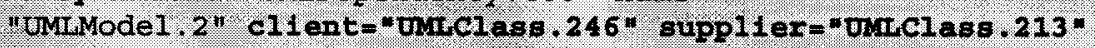
< onu : Dependency $\mathrm{xmi}$. Id= "UMLDependency. 407 " name=" "

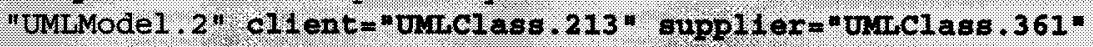

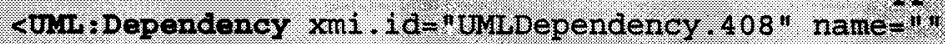

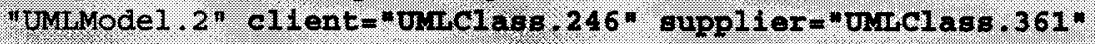
<uma : Dependency xmi. id= "uMLDependency. 409" name-" "

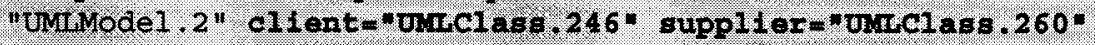

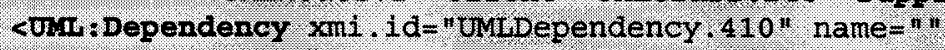

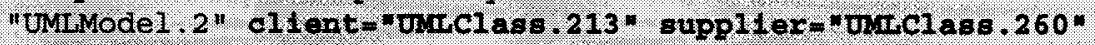

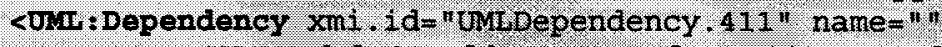

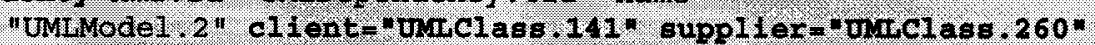

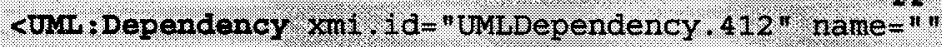

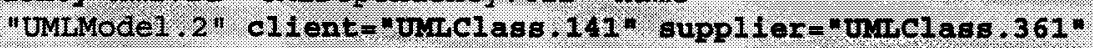
<UA, Deperdency xni. $1 \mathrm{~d}=$ "UMLDependency. 413 " name=" "

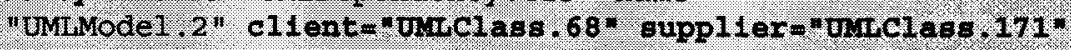
<uki: Dependency xil. $1 \mathrm{~d}=$ "umLDependency. 41.4 " name="

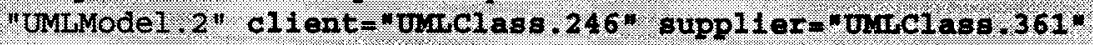

Figure 6.8 UML class diagram for the CAL parser in XMI

relationship has a supplier and a client. First we load the UML model of the Class Diagram using the XMI file for the CAL parser in VCAL, by using the menu File-->Load Model. While loading the model, VCAL also populates the CAL data 
structure (ADAG). The CAL data structure is kept in VCAL memory for efficient use. Figure 6.9 displays three CAL files with different CAL actions and operations. Now we describe how VCAL interprets and executes these three CAL files:

(a) addClass_CodeGen.cal: We open the file addClass_CodeGen.cal listed in Figure 6.9 (a) in the VCAL editor to run this file. There is one pre condition, three actions and three post conditions in the first operation context addClass in this file. The second operation context, viewDag, consists of only one CAL operation view. To run this file we press the Run button on the VCAL Toolbar. VCAL parses the file using the C++ CAL parser, which generates the AST in XML. The AST is then interpreted and executed by the CAL interpreter. The CAL interpreter generates the ADAG of the model. Then it creates a class CodeGen and links it to three classes, $A S T$, Error and $L G$. These three classes become the parent of CodeGen class, or we can say that CodeGen becomes the descendant of these three classes in the ADAG. After this, the CAL interpreter executes the three post conditions, which check if CodeGen has become the descendant of these three classes. Then VCAL displays the ADAG by executing the CAL operation view in the context Model::viewDag, as shown in Figure 6.10 (a). The complete ADAG is displayed in Figure 6.7 without the CodeGen class. We can now apply one of the ADAG view filters, View Ancestors, from the toolbar on the CodeGen. This filter displays all the ancestors of only the CodeGen, as shown in Figure 6.10.

(b) removeLinks_CodeGen.cal: As the name suggests this file, which is shown Figure in 6.9 (b), removes the links (dependencies) of one of the classes 
CodeGen from the ADAG. Before removing the links we check if CodeGen has no decendants (dependents). Then we remove CodeGen from the descendants of $A S T$, Error and $L G$ class by removing the links from $A S T$ to CodeGen, Error to CodeGen and $L G$ to CodeGen. In the last three post conditions in Figure 6.9 (b) we verify this operation by checking if the CodeGen is not one of the descendants of AST, Error or $L G$ class (i.e. dependencies removed).

Table 6.3 Test matrix for the case study

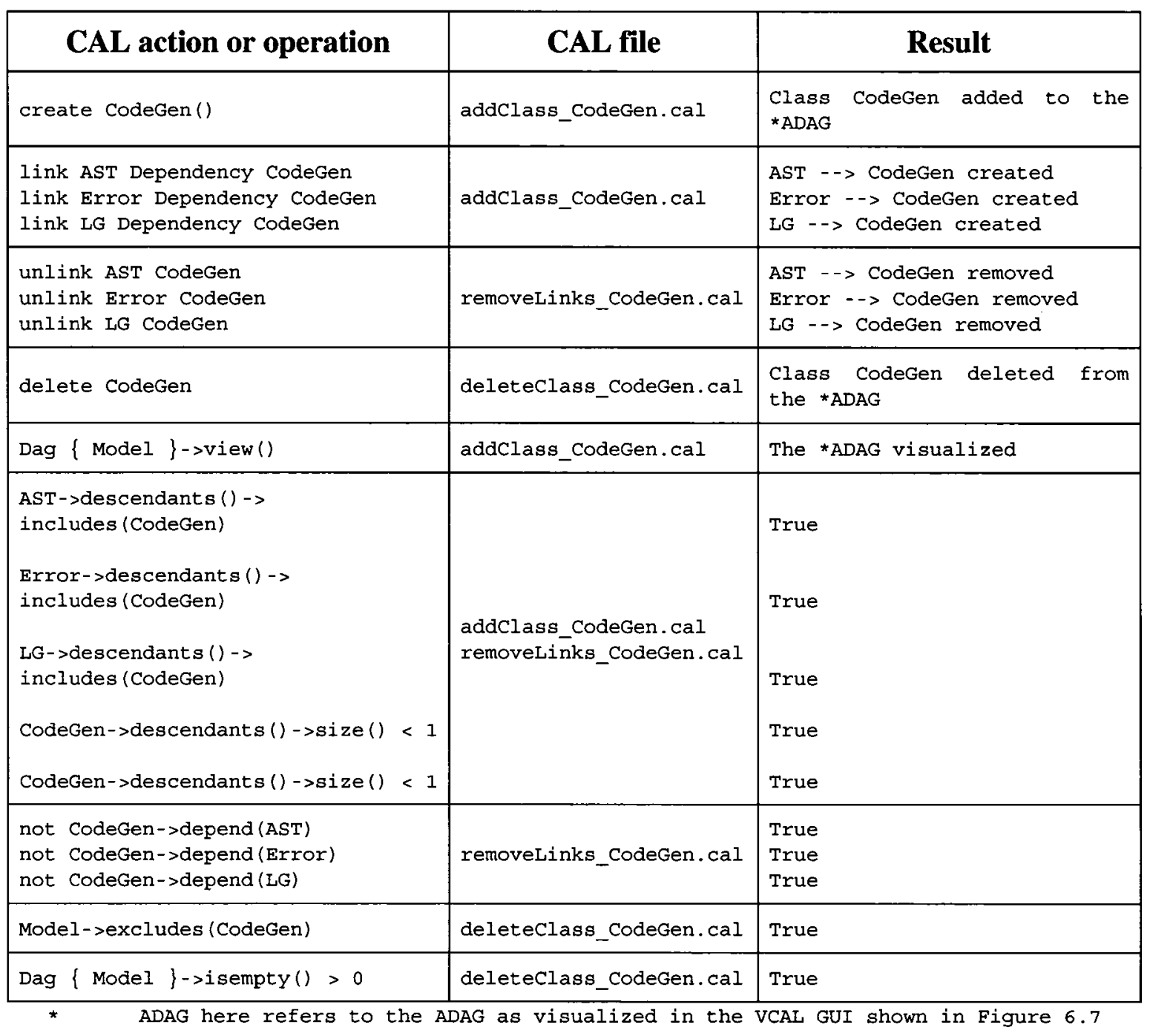


(c) deleteClass_CodeGen.cal: In this file, which is shown in Figure 6.9 (c), we delete the CodeGen class from the ADAG. Before deleting we check if the ADAG is not empty and the CodeGen has no descendants. After deleting, we confirm that the ADAG does not contain the CodeGen class.

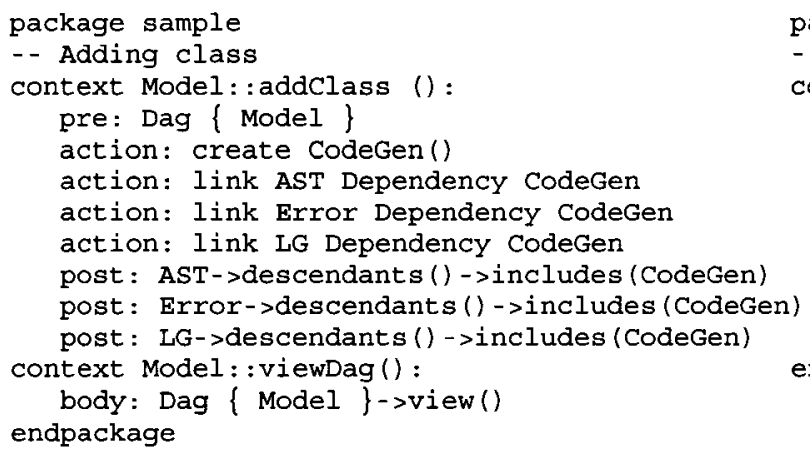

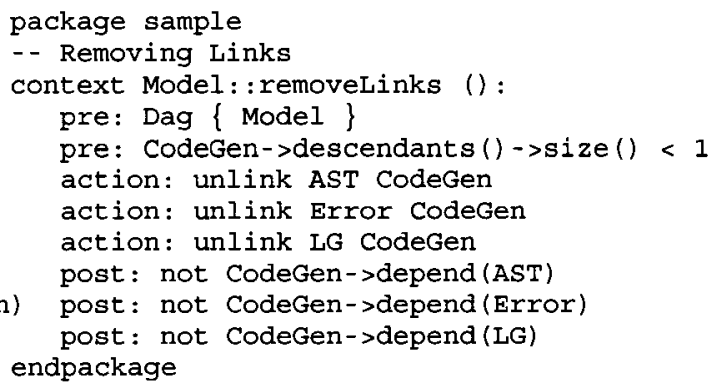

(b) removeLinks_CodeGen.cal

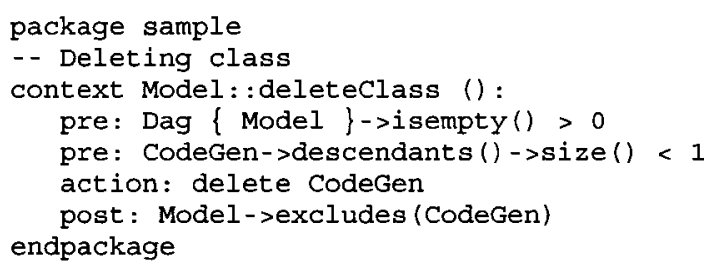

(c) deleteClass_CodeGen.cal

Figure 6.9 CAL files with different $\mathrm{CAL}$ actions and operations 


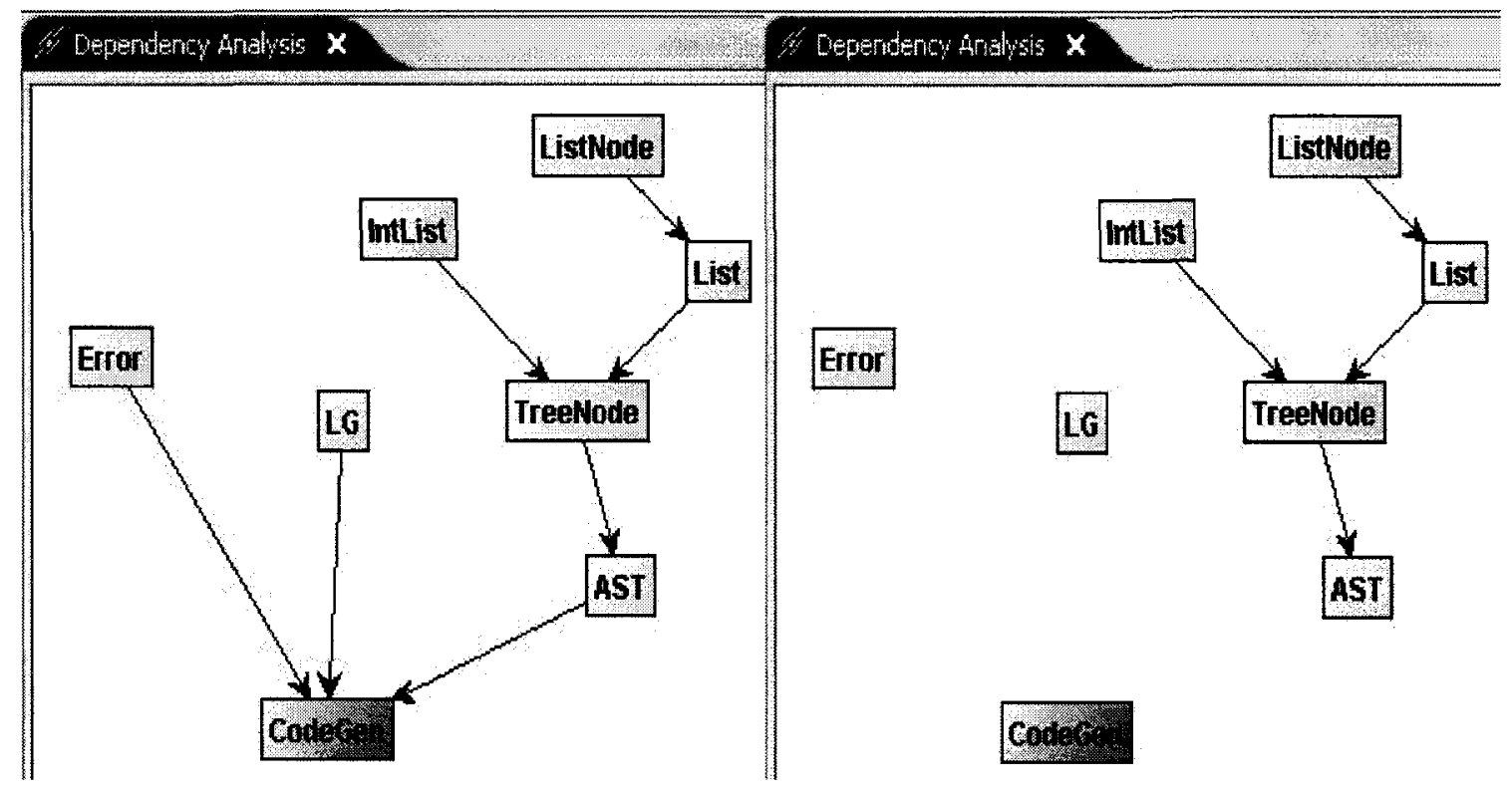

(a) addClass

(b) removeLinks

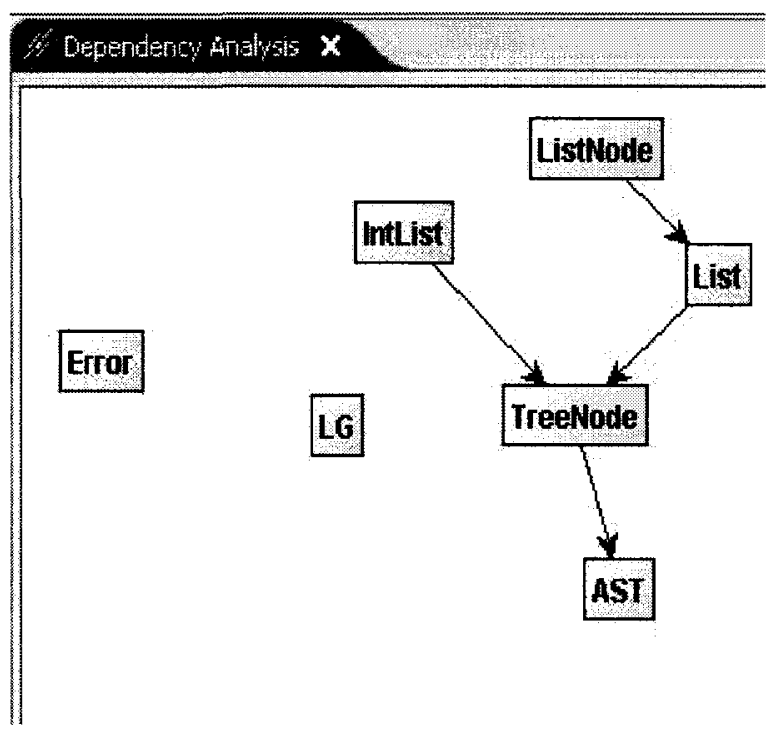

(c) deleteClass

Figure 6.10 VCAL GUI showing the ADAG after executing different CAL actions and operations, shown in Figure 6.9 


\section{Chapter 7}

\section{Conclusion}

A software system starts changing before it is being delivered. More time is spent on changing the software system than on its development. Of all the software processes, evolution is the most expensive. There is a need to evolve the system from the beginning. To achieve this, software evolution must be integrated into the design (model) of software systems. There are two issues that our work examines and resolves. One of them is the support of software evolution in modeling languages. The other issue is the automation of model evolution. A solution is provided in the form of a modeling language CAL (Constraints with Action Language).

Finding all the dependency relationships whether direct or indirect, among UML (Unified Modeling Language) elements in a model results in a large number, making it impractical to verify, manage and analyze them manually. Due to the lack of support of software evolution management in modeling languages and tools, it is highly desirable to integrate such support into them. Our research indicates that none of the previous efforts, either commercial or academic, support automation of software evolution in a modeling language. This thesis proposed a novel approach for the automated management of software evolution in models. The proposed approach is highly portable, flexible and easy to use. OCL (Object Constraint Language) is an expression language and is guaranteed to be without side-effect. This means that the expressions do not change 
anything in the model of the software system. OCL can be used to return values. We can say that OCL expressions are like conditions that determine an action, rather than instructions that carry out an action. Using CAL, a modeler can write a constraint using the same language used for actions. Before describing our research contributions, limitations and future research possible with $\mathrm{CAL}$, we first list some of the characteristics of CAL:

$\checkmark$ Complete extension of OCL, so constraints can be used with actions. Hence, CAL combines both declarative and imperative programming paradigms;

$\checkmark$ Provides automation for dependency analysis;

$\checkmark \quad$ Easy to learn. Abstracts the details so even a non-programmer in a business environment can use it effectively;

$\checkmark$ A well-defined grammar in standard EBNF [1] form;

$\checkmark$ Formally specified in TLA+ [50] for better comprehension, implementation and verification;

$\checkmark$ A CAL parser implemented in $\mathrm{C}++$, which can easily be integrated into other modeling tools.

The major contribution of this thesis is that we have extended OCL and defined a new language, CAL that can be used for model evolution. CAL gives a user (modeler) the ability to use constraints with actions on models, and provides the user with tools to automate model evolution. A new set of Collection directed acyclic graph (DAG) has been added to the CAL library for automating dependency analysis. CAL contains a 
small set of constructs but is powerful enough to be used efficiently for typical software evolution management operations like impact analysis, correction, improvement and enhancement of models.

Since CAL has its own well-defined data structure and type system, it is independent of other modeling tool's API's, data structure and type system. This makes CAL highly interoperable, portable and flexible.

\subsection{Limitations}

The solution proposed and the prototype implemented in this thesis has the following limitations:

× The CAL data structure (ADAG) can only process acyclic dependency relationships among UML model elements. It is easy to remove cyclic dependency relationships, but how can we add them to the ADAG (Annotated Directed Acyclic Graph) for impact analysis? Hence, more research is required to find methods and techniques (As transformation and mapping rules) to remove and add these cyclic dependencies to the ADAG.

× VCAL loads the complete UML model, but can only process a UML model of the Class Diagram. Because in this thesis, transformation rules that are used in VCAL to transform a UML Diagram to the ADAG, are only defined for a UML Class Diagram.

× The CAL interpreter in VCAL does not provide interpretions for all the CAL instructions.

\subsection{Future research}

Model driven architecture (MDA) [11,47] uses model transformation language to 
transform a model from platform independent model (PIM) to platform specific model (PSM). Executable UML $[9,16]$ takes the concept of MDA further. It removes the PSM from MDA and directly generates code that can execute. The CAL data structure (ADAG) can be used to propagate change automatically to the transformed model or to the generated code so that when the software modeler makes a change to the model, the model compiler is able to detect which elements of the model have been modified and then can determine what minimal elements need to be recompiled or retransformed and in what order.

To use CAL for MDA or executable UML, we first need to define rules and mapping for translating PIM into the CAL data structure. As the standard for Executable UML is still Work In Progress by OMG, using CAL for executable UML can be a future research area.

CAL can also be extended for use as a full action language in MDA. To do this we need to define rules for translating CAL actions to code for different programming languages. For example, current CAL actions, operations and its data structure can be translated automatically to Java language, as is done manually in this thesis.

How to apply CAL to a UML Statechart Diagram for behavioral model evolution? A Statechart Diagram is a directed cyclic graph and can be transformed (filtered) to a DAG for dependency analysis after removing cycles from the graph. A state in a Statechart Diagram is either an operation, or a statement in some operation, in a Class Diagram, so a dependency between states of different classes in a Statechart Diagram can be mapped to the Class Diagram. We need to define these mapping rules so that we can add a dependency from a Statechart Diagram to the CAL data structure. 
The implementation of a full CAL interpreter is beyond the scope of this thesis. A full CAL interpreter and compiler could be another promising research area.

This thesis has defined rules for translating a UML Class Model to the ADAG. VCAL uses these rules to load a UML model from an XMI (XML Metadata Interchange) file and translates the UML Class Model to the CAL data structure. VCAL also applies changes and filters to the ADAG. This ADAG is then saved by VCAL as an XML file. VCAL does not map this XML file of the changed ADAG to the XMI file of the UML model. This XML file needs to be translated and integrated into the UML model, so we need to define rules for this translation and integration, and we need to verify this translation and integration with the changes made in the ADAG. 
Appendix A

\section{CAL Grammar}

TITLE: CAL (Constraints with action language) Grammar in EBNF [1]

AUTHOR: Shahid Alam

DATED: July 31, 2006

REVISION: 3.0 (January 11, 2007)

\section{DESCRIPTION:}

The grammar can be defined by a 4-tuple $G=(T, N, P, S)$ where

$\mathbf{T}=$ set of terminals

$\mathbf{N}=$ set of non-terminals

$\mathbf{P}=$ set of production rules

$\mathrm{S}=$ an element of $\mathrm{N}$, a distinguished starting non-terminal

Section 9.3 "Concrete Syntax" and section 12.13 "Concrete Syntax of Context Declarations" in the OMG document [2] describes the grammar for OCL 2.0. It is very hard to write a parser for this grammar. As mentioned in the specification of OCL 2.0 in [2] and is quoted below:

"The grammar in this chapter might not prove to be the most efficient way to directly construct a tool .... Also, error correction or syntax directed editing might need hand optimized grammars".

This document describes the grammar for CAL. An optimized parser has been implemented manually to validate the grammar. This grammar is an extension to OCL 2.0 mentioned in above sections, keeping in view the abstract syntax of OCL 2.0 and action semantics [5] of UML to add actions to constraints. The grammar uses the EBNF syntax, where "|" means a choice, "?" means optional, "**" means zero or more times and " + " means one or more times. Line Comments start with "-". Terminator symbol is ";". Terminals are enclosed in single quotes. The start symbol is "file". It has been optimized for $L L(1)$ parsing, to implement a predictive parser [3].

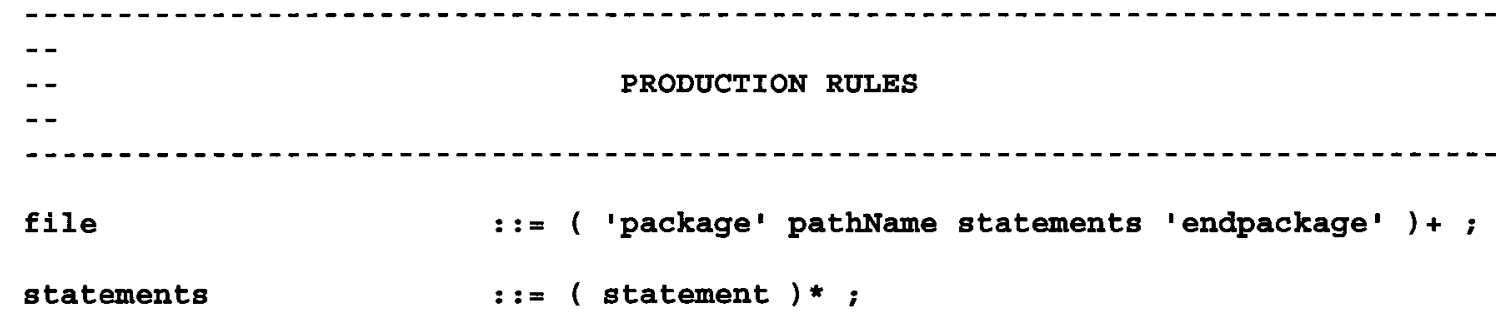




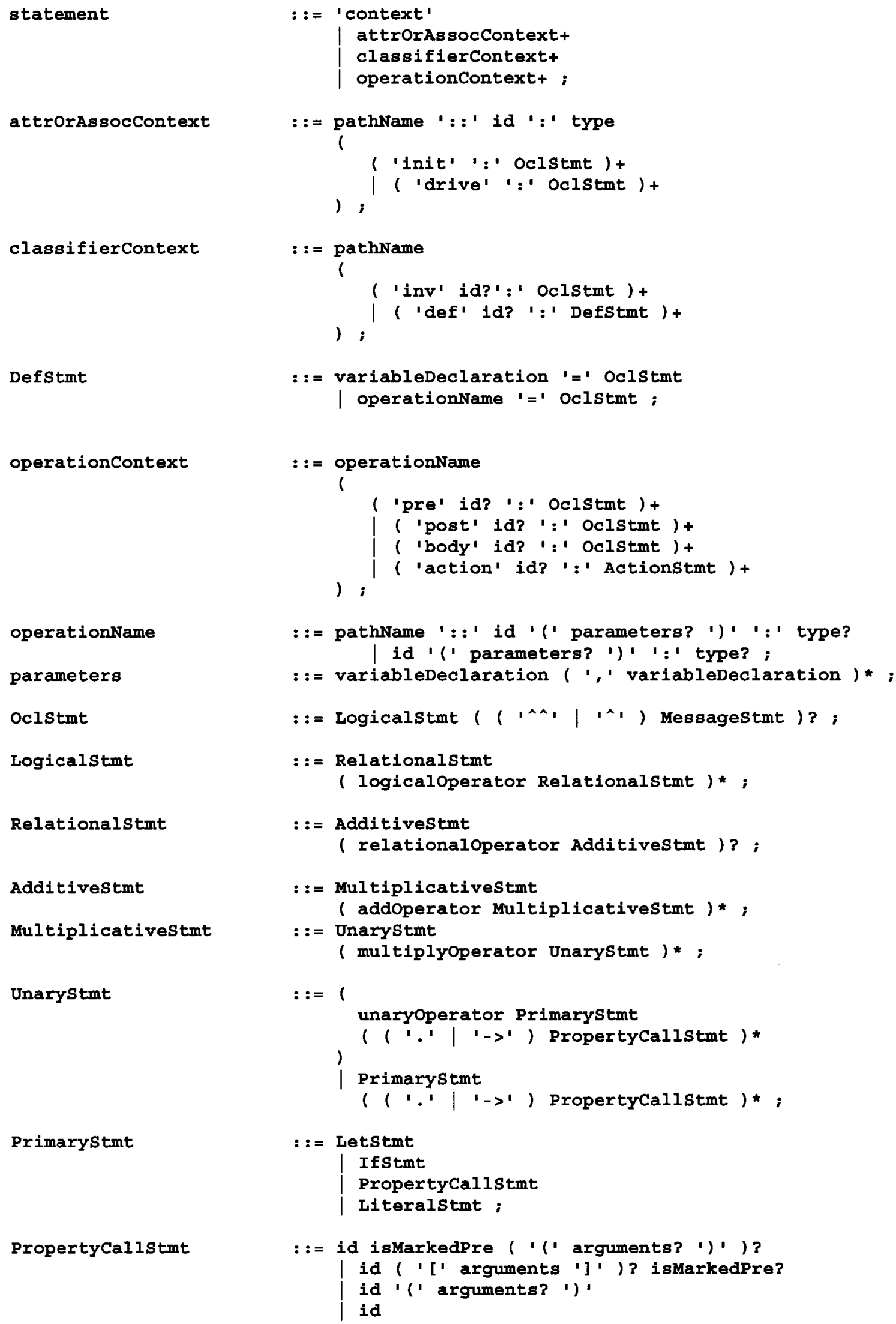




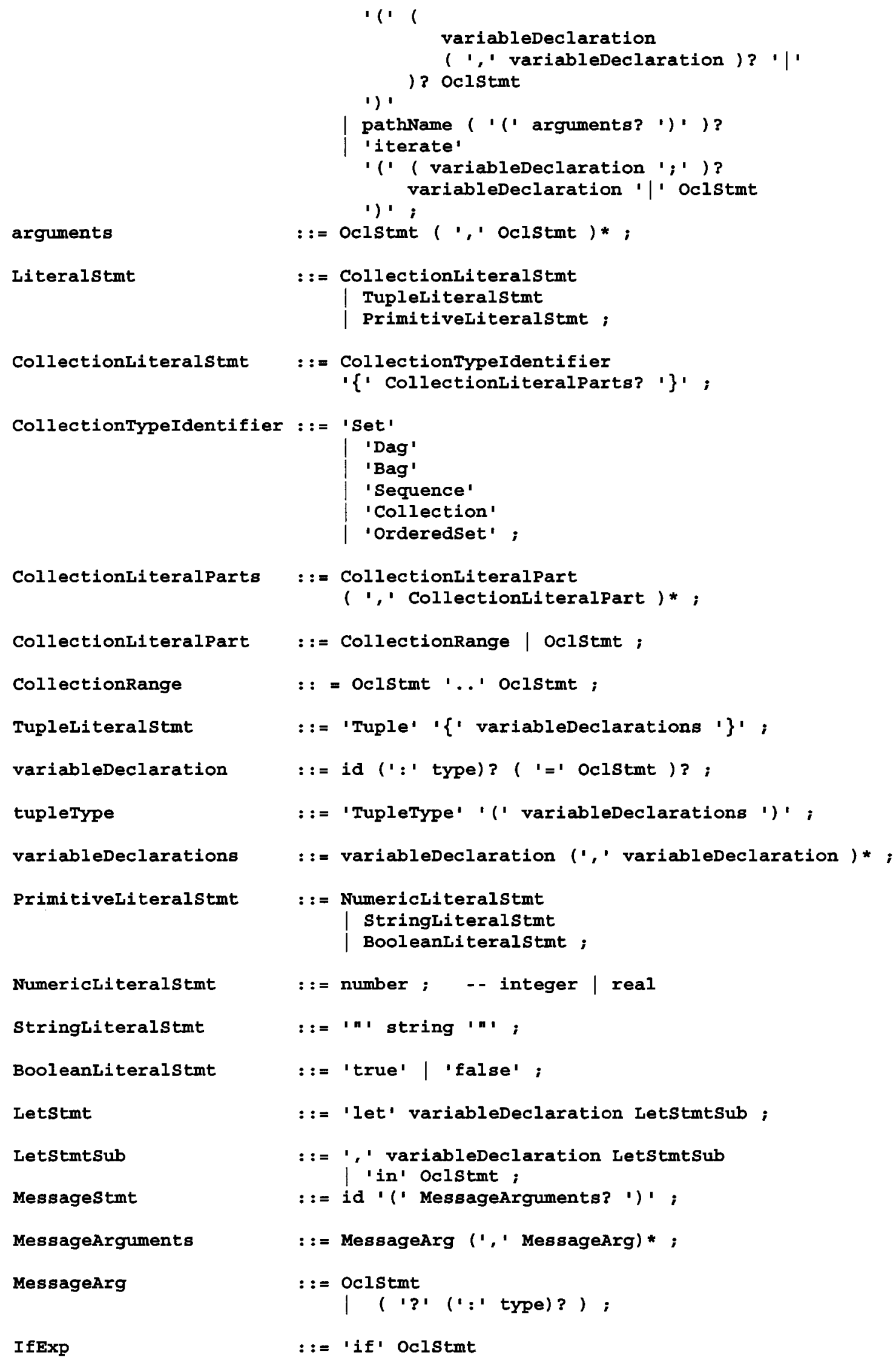




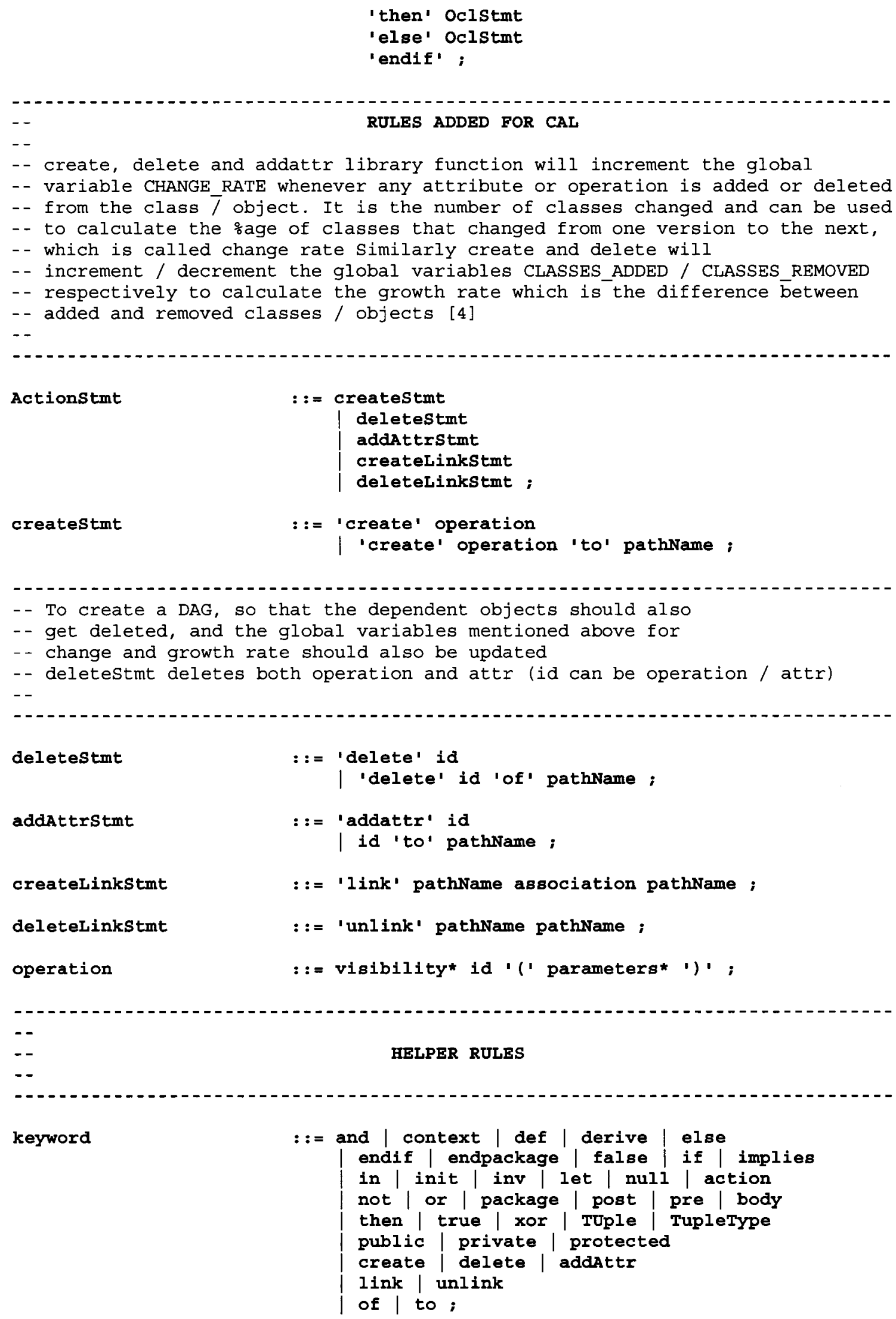




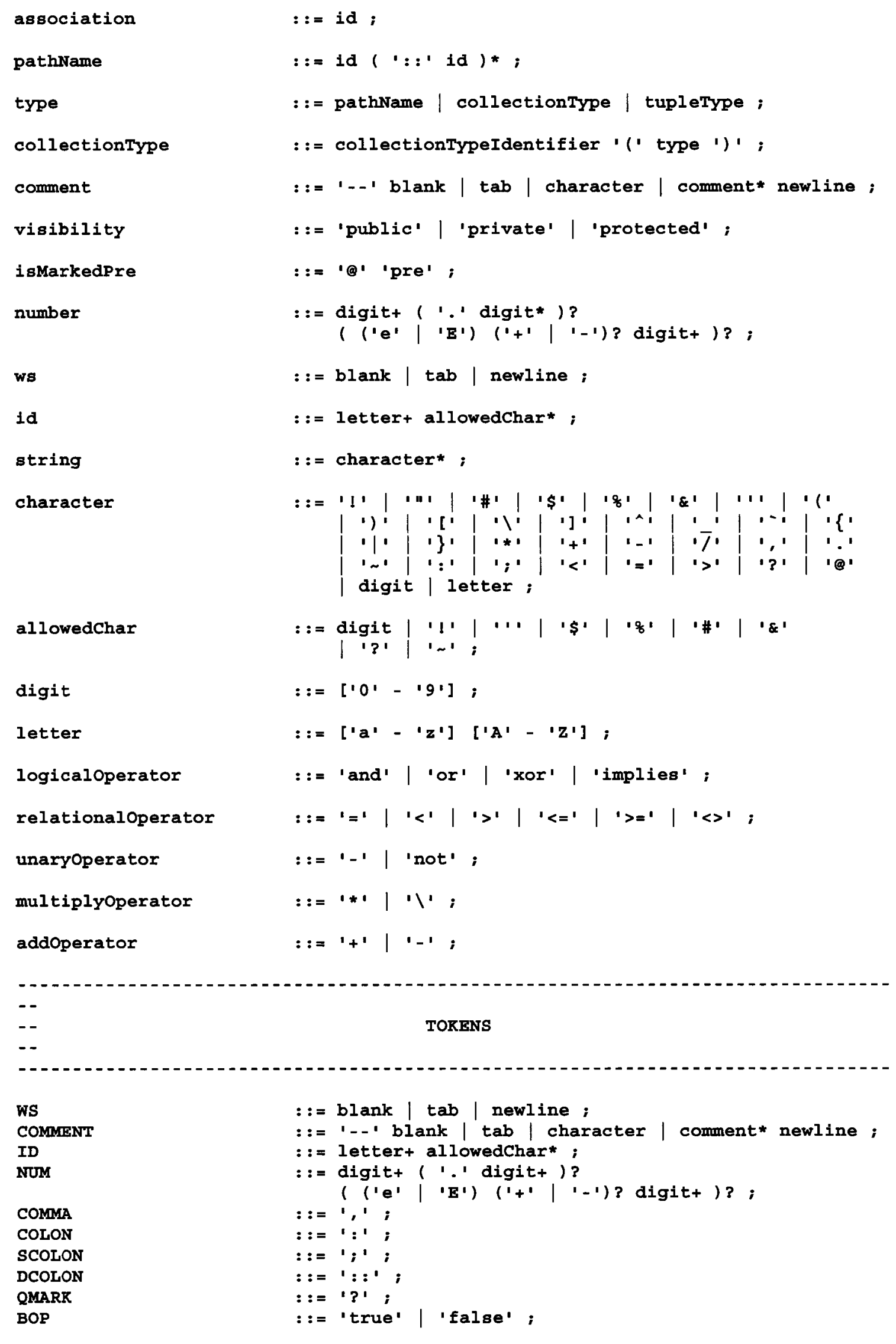




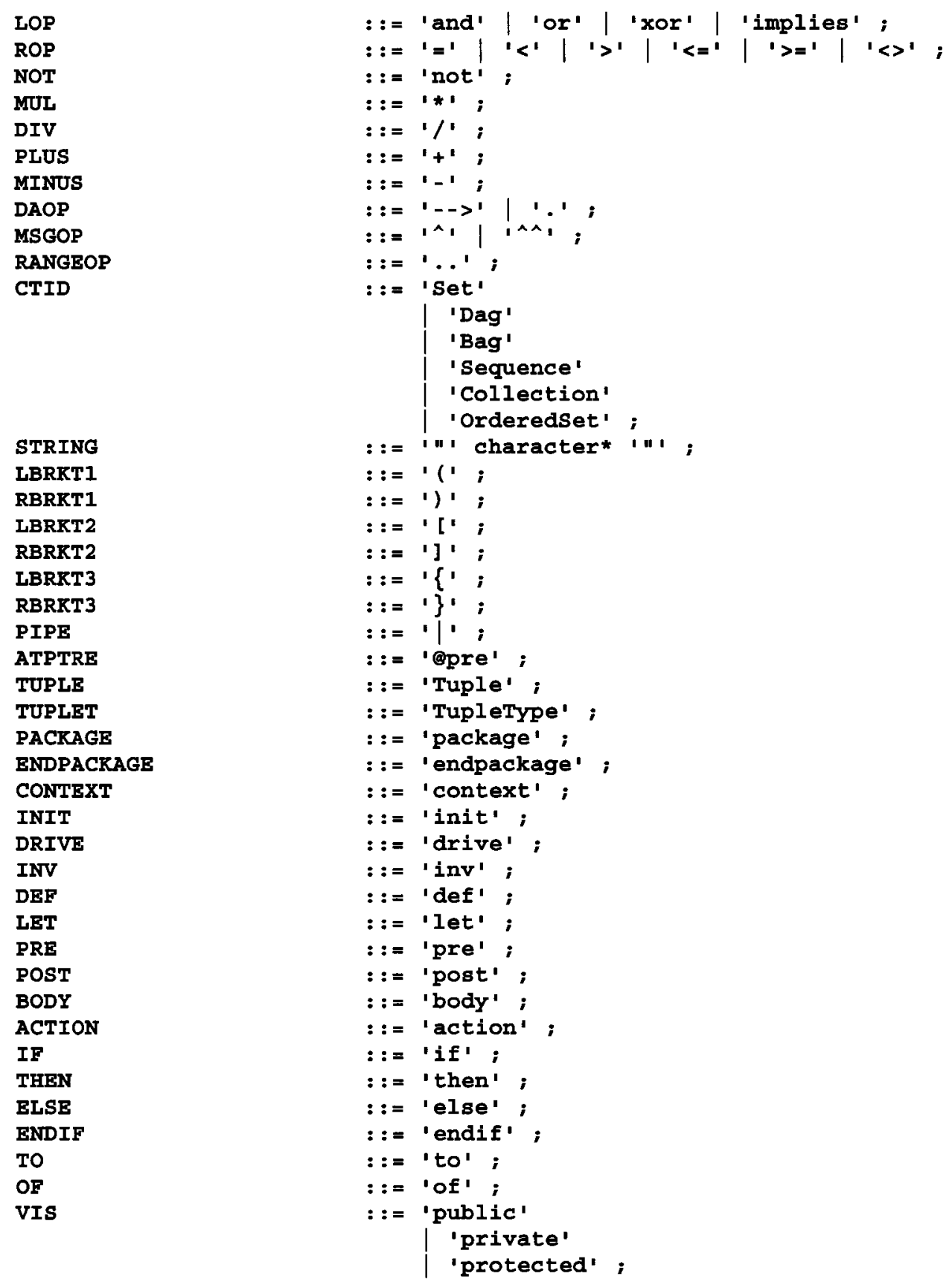


Appendix B

\section{CAL Parser's Finite State Machine for Lexical Analysis}

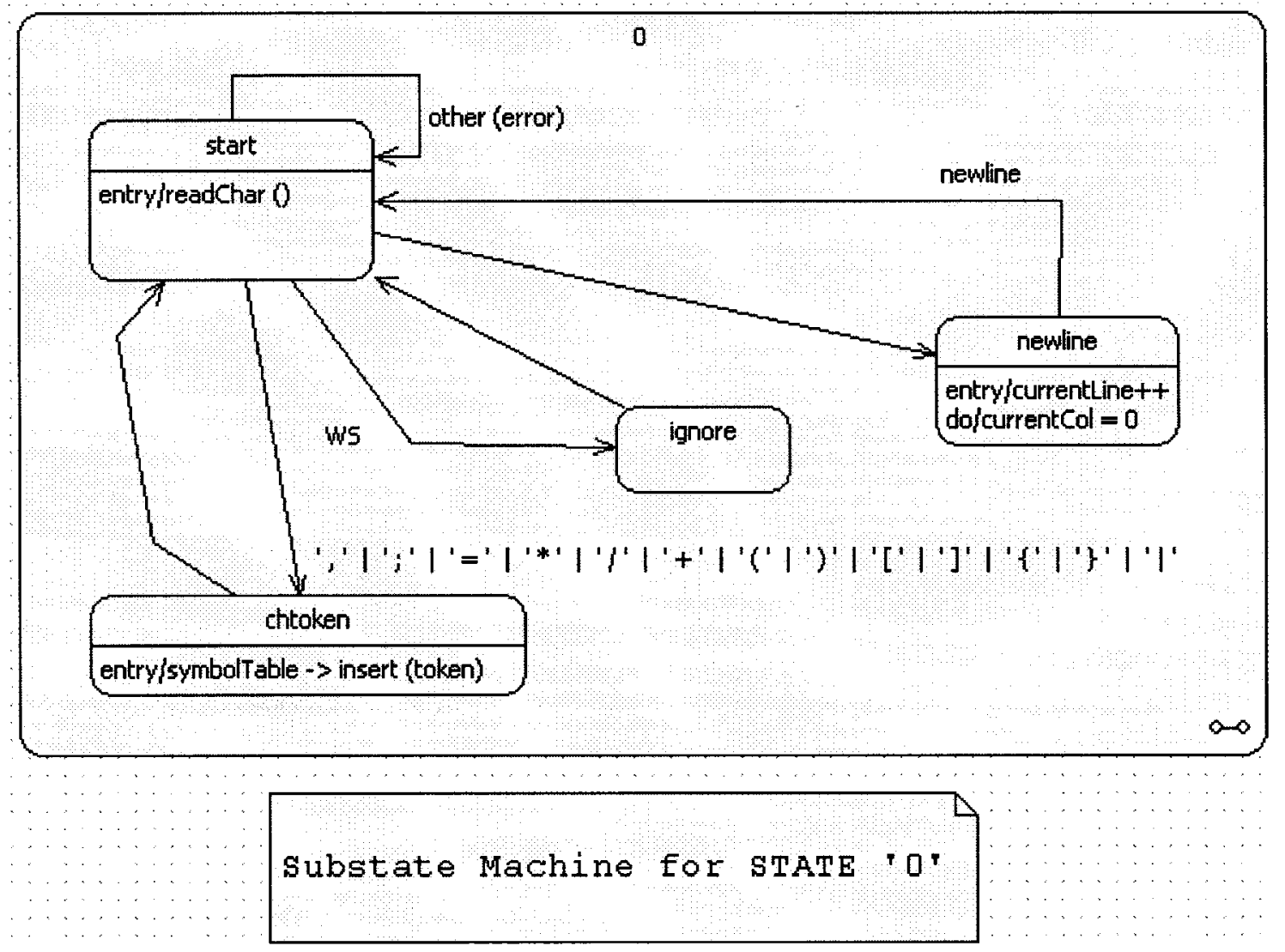




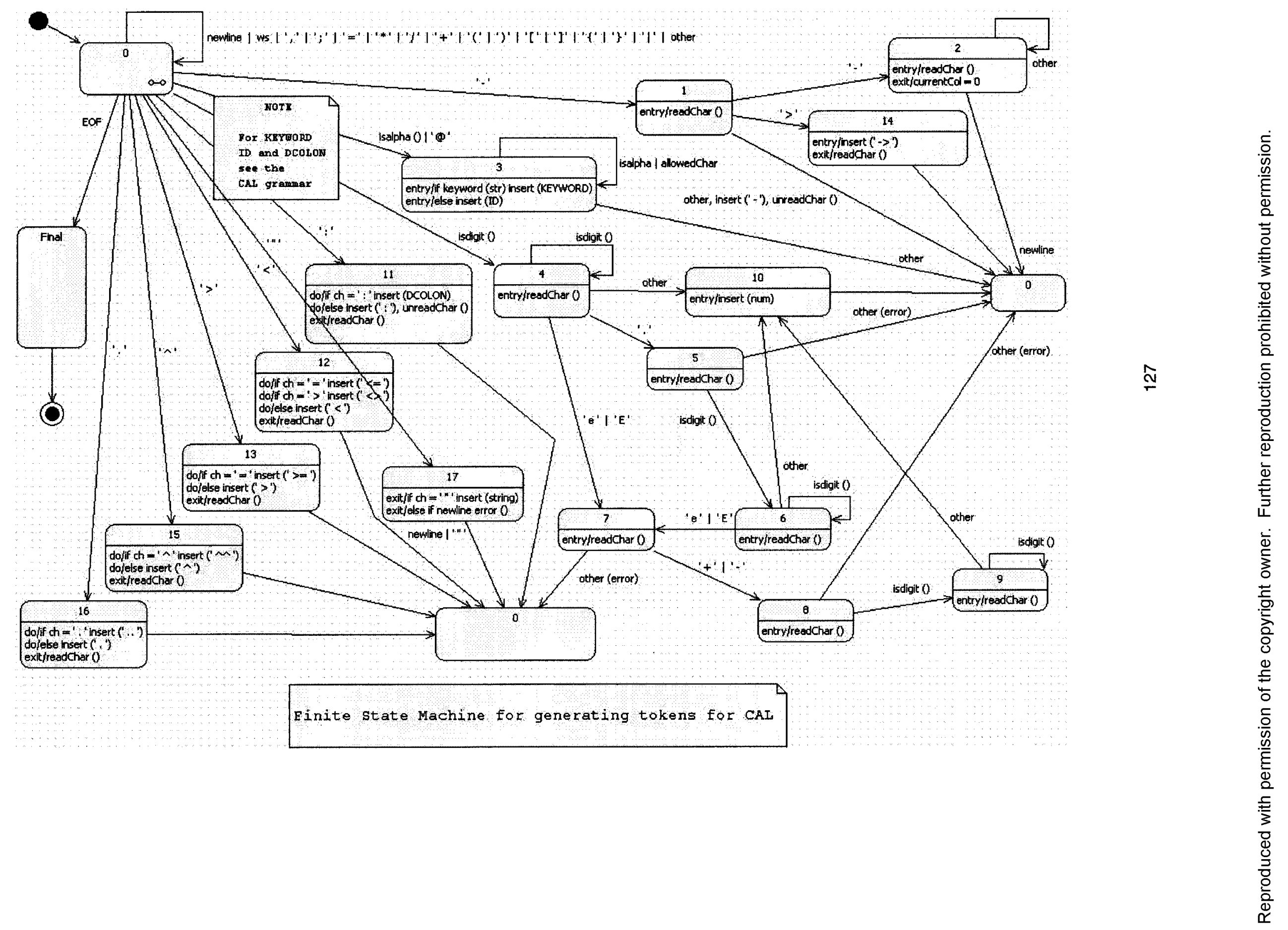


Appendix C

\section{CAL Parser's Syntax and Parsing Tree}

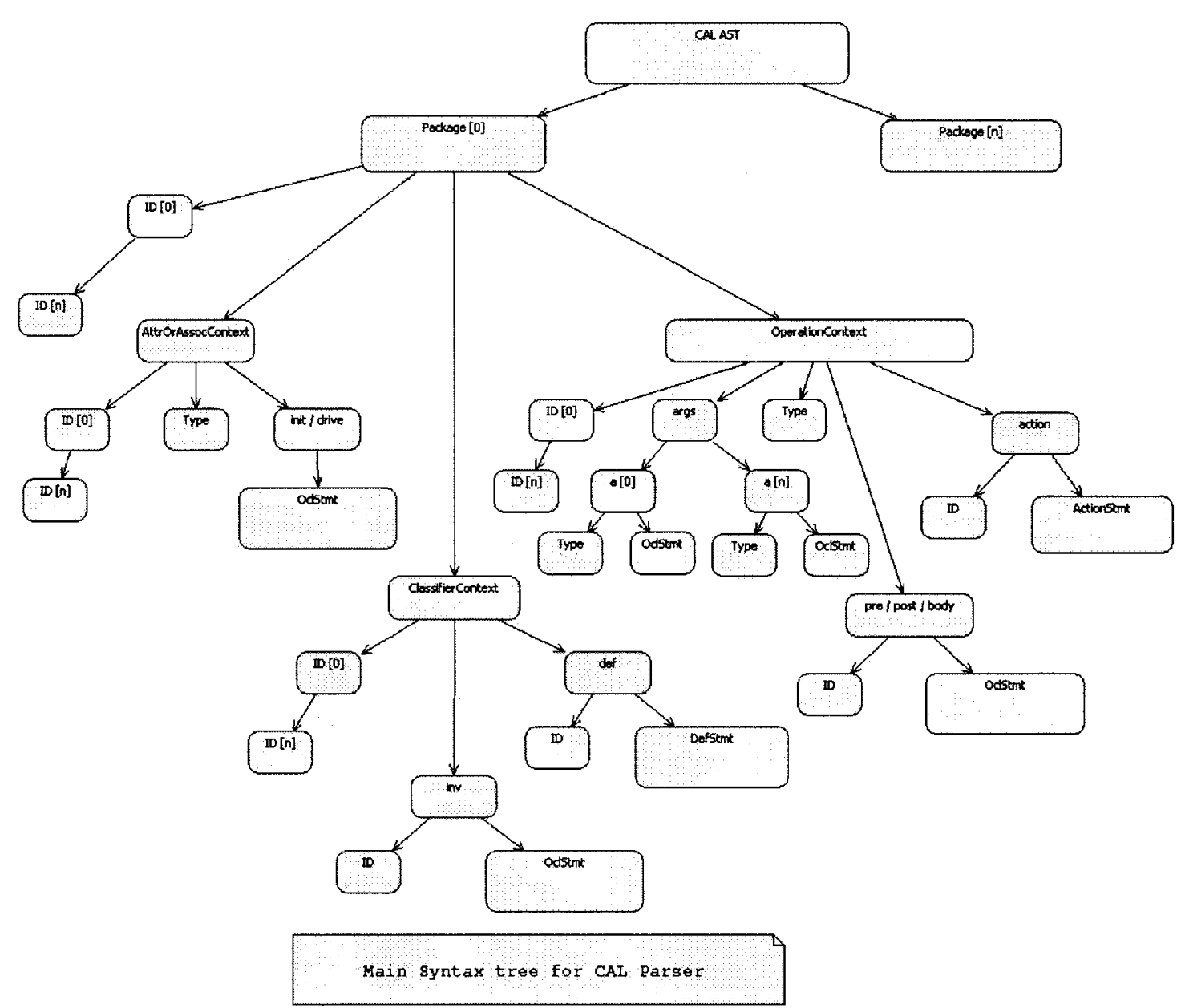



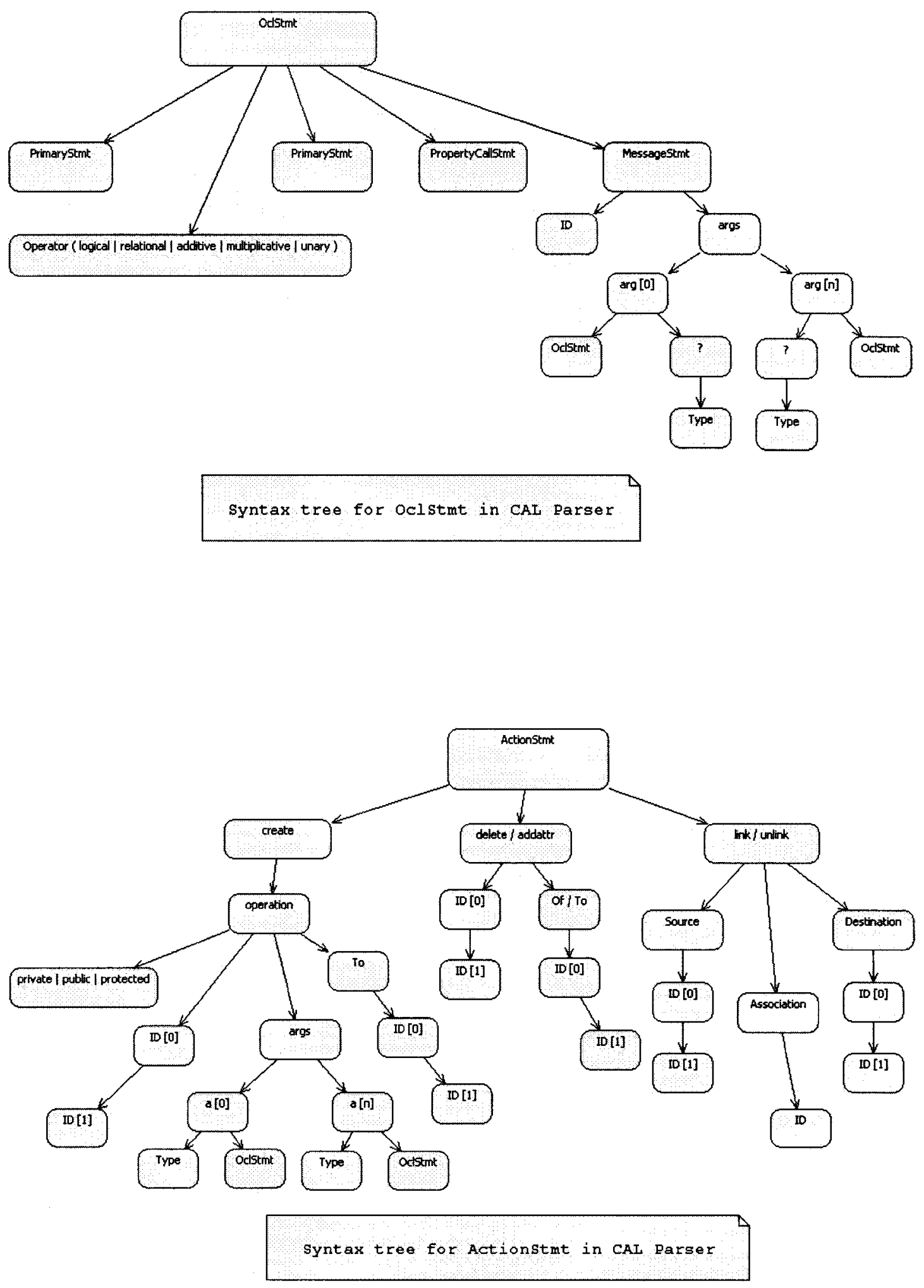


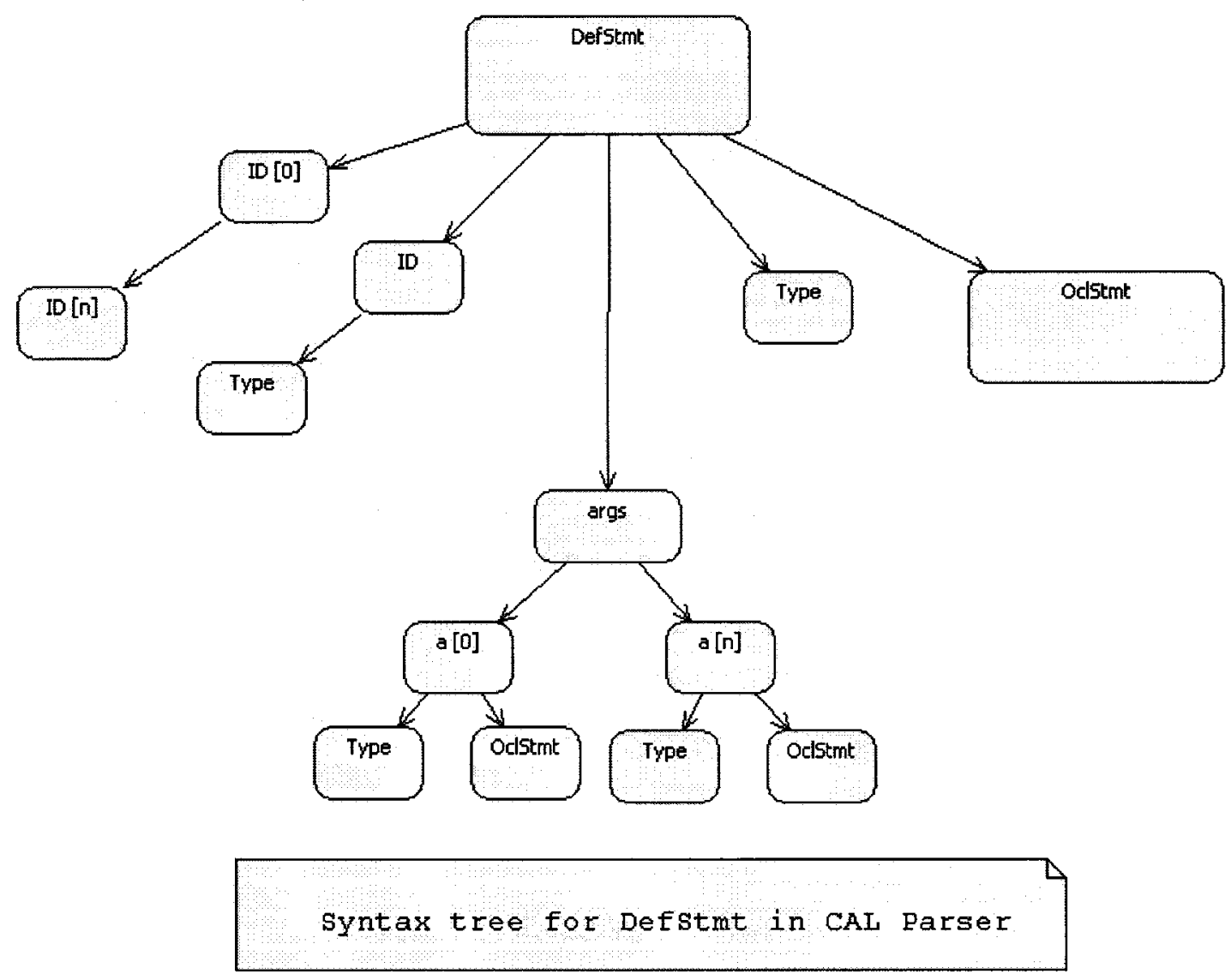




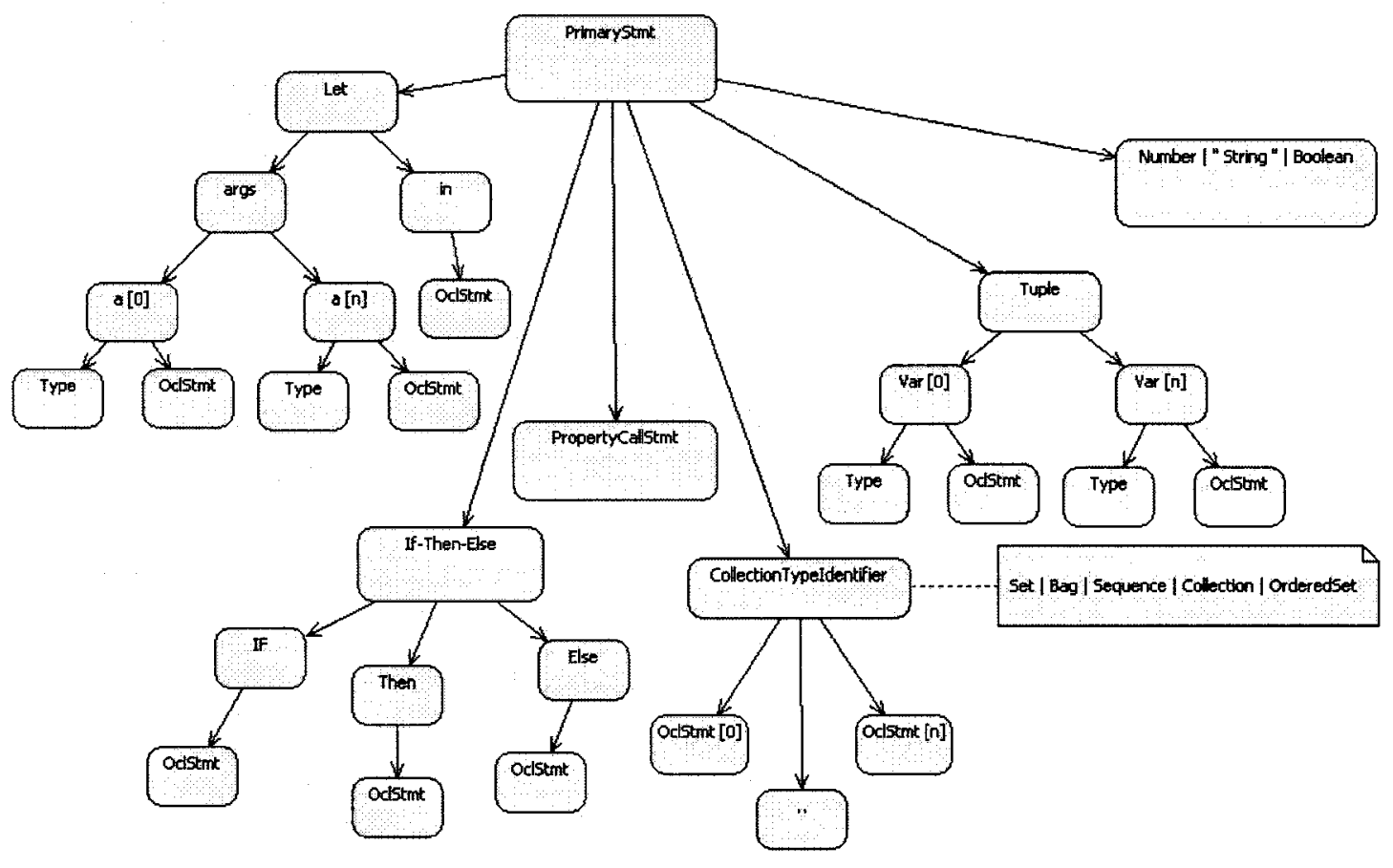

Syntax tree for Primarystmt in CAL Parser 

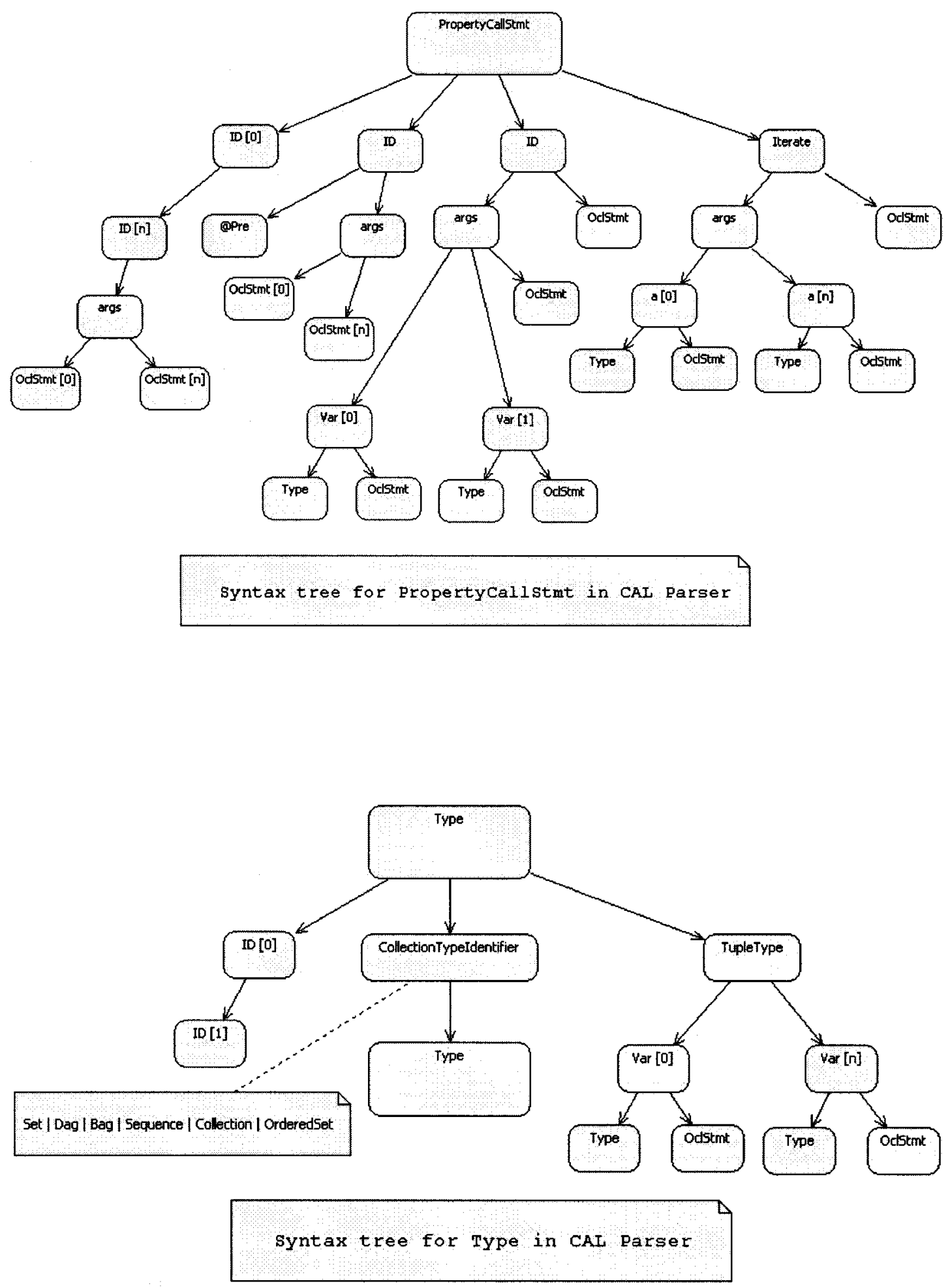


\section{CAL Parser's UML Class Diagram}

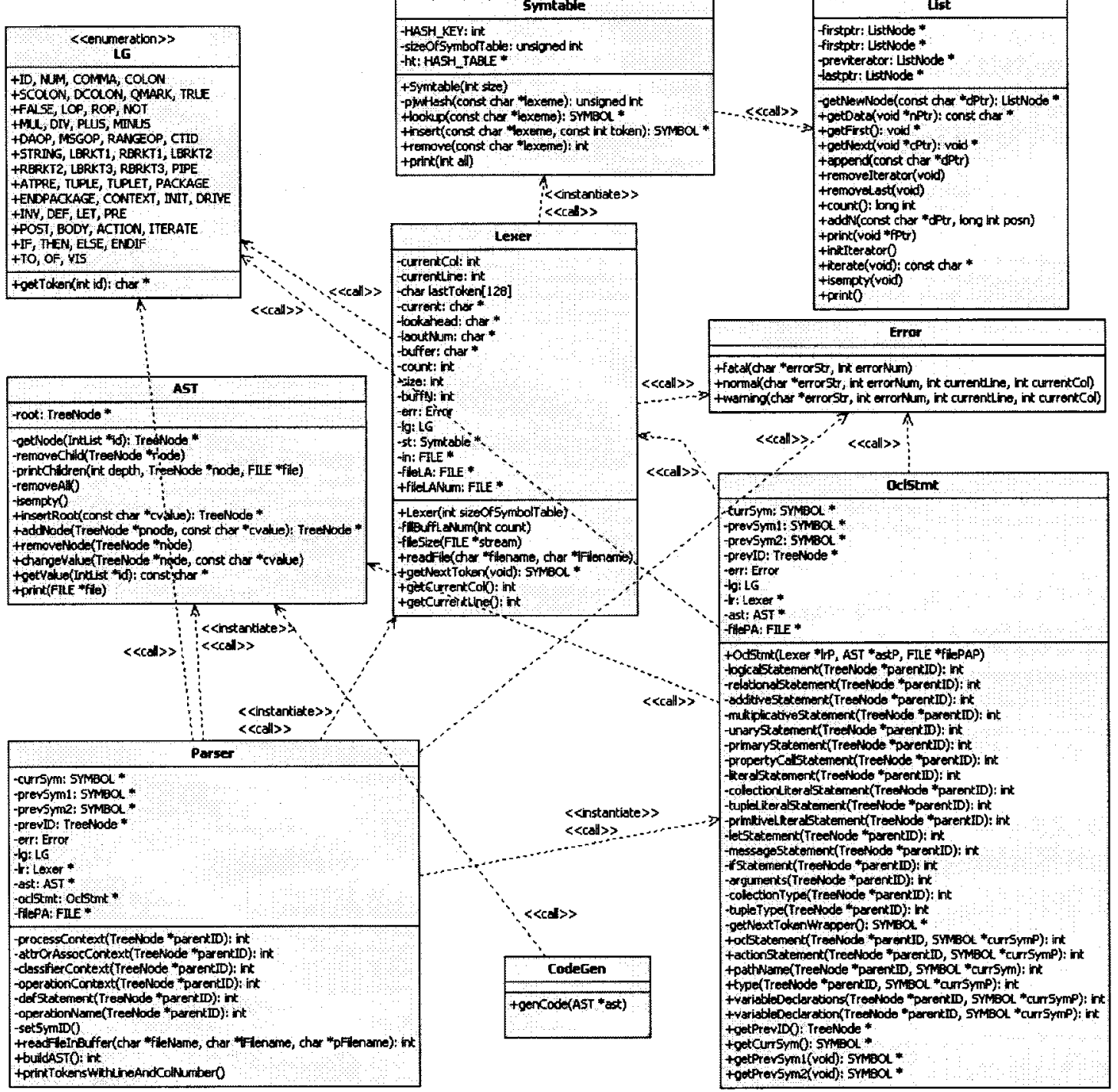




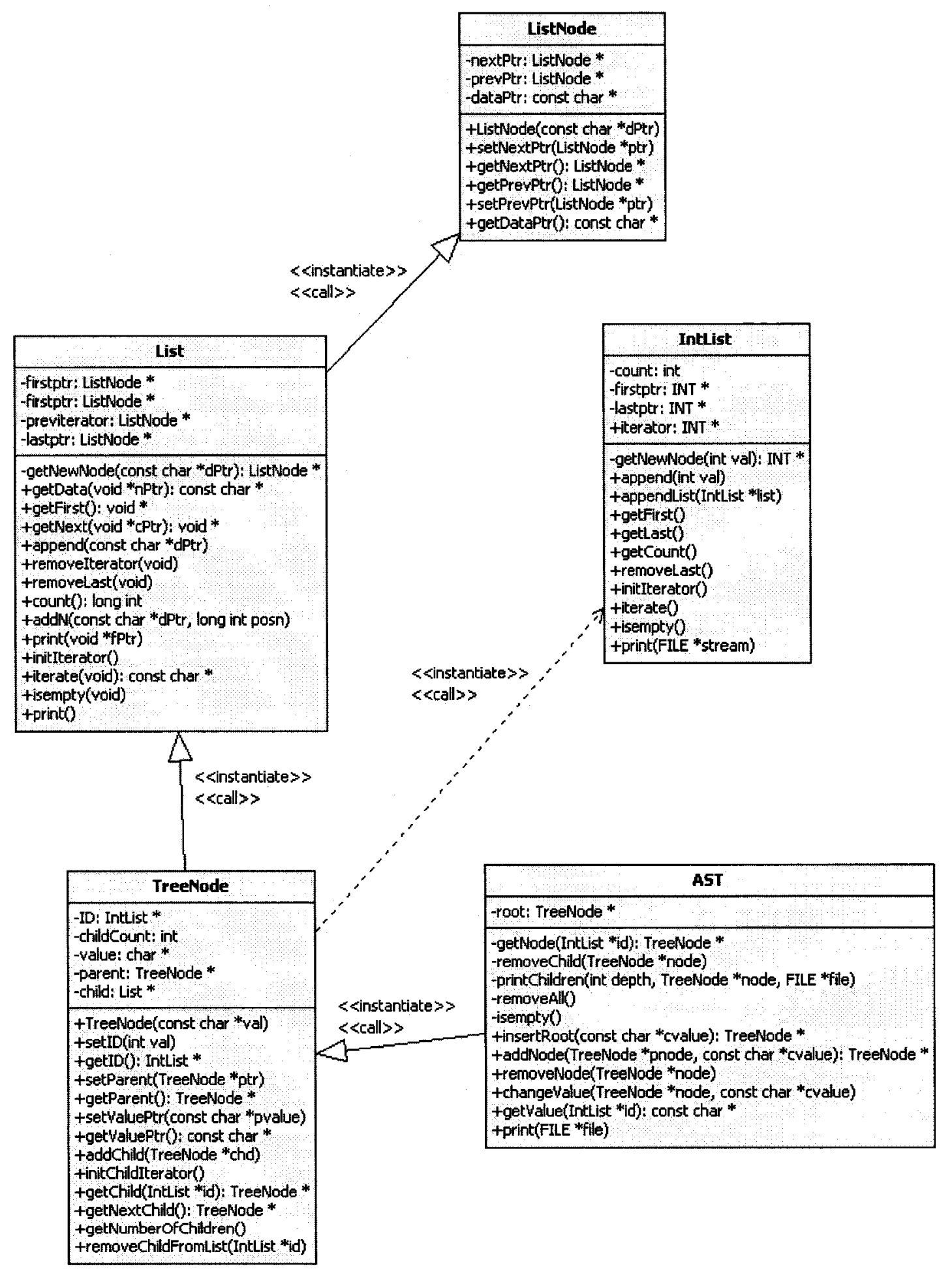


Appendix E

\section{CAL Specifications (ASCII Version Using pluscal, Translation not Shown) in TLA+ with Configuration File and TLC Output}

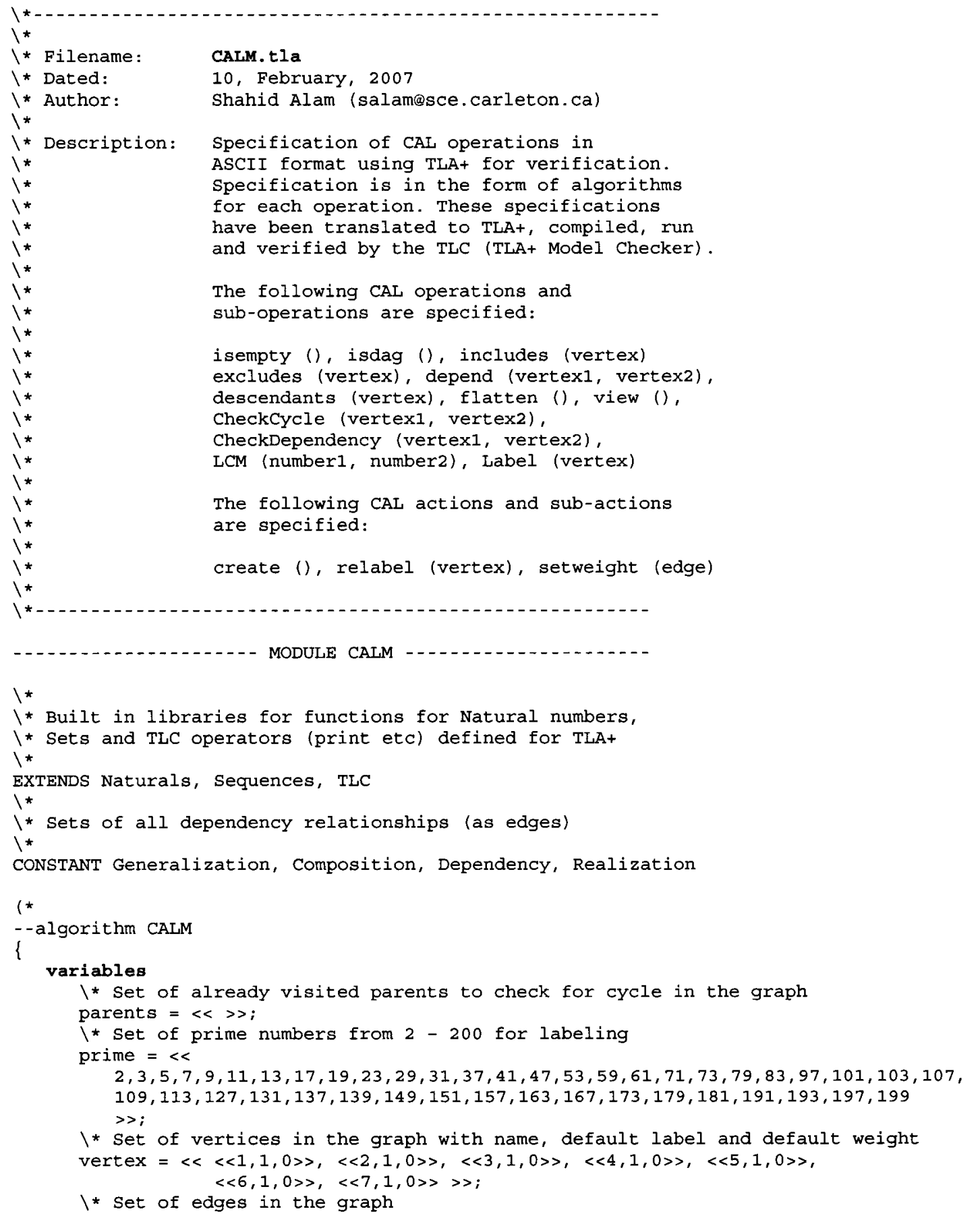




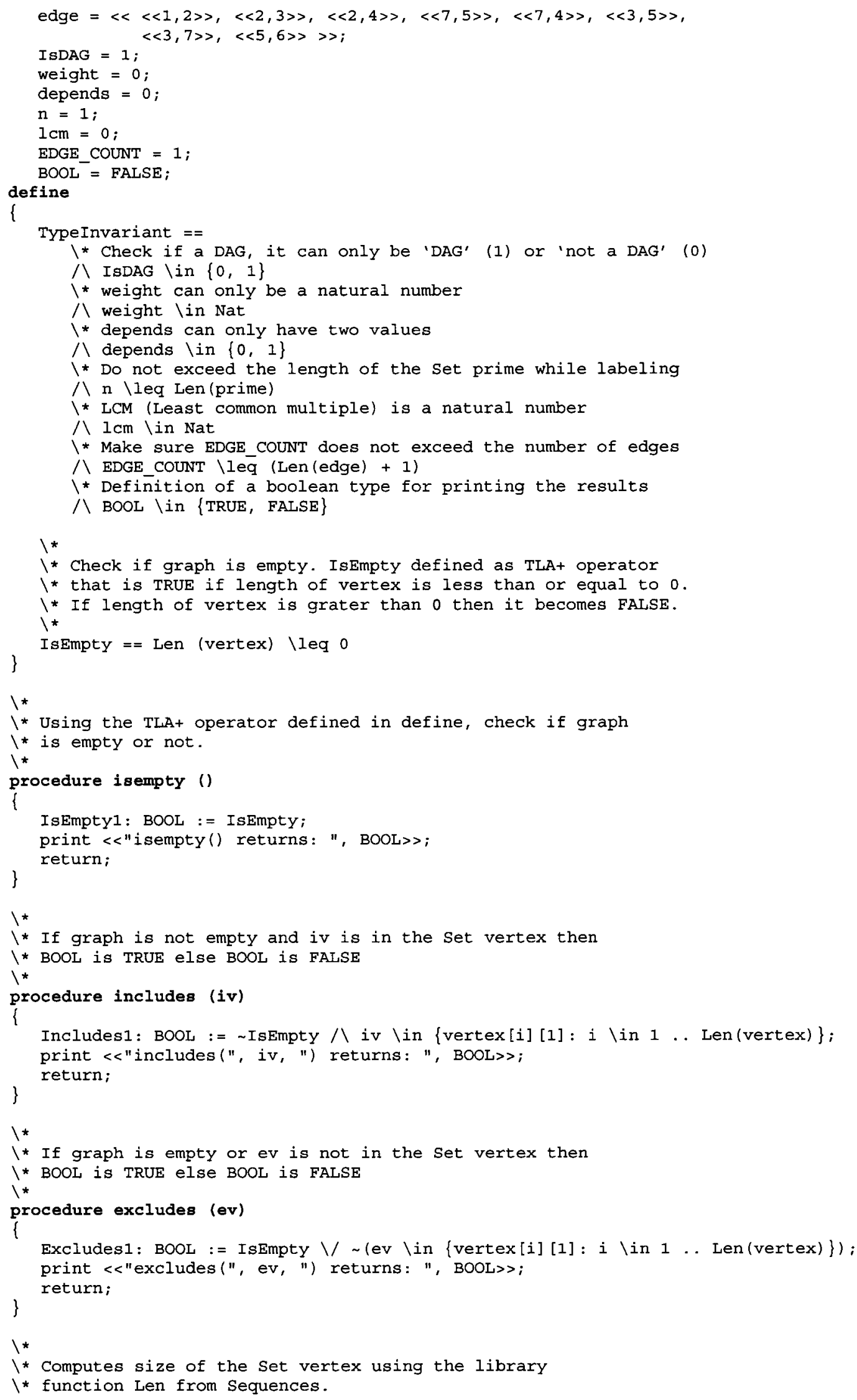




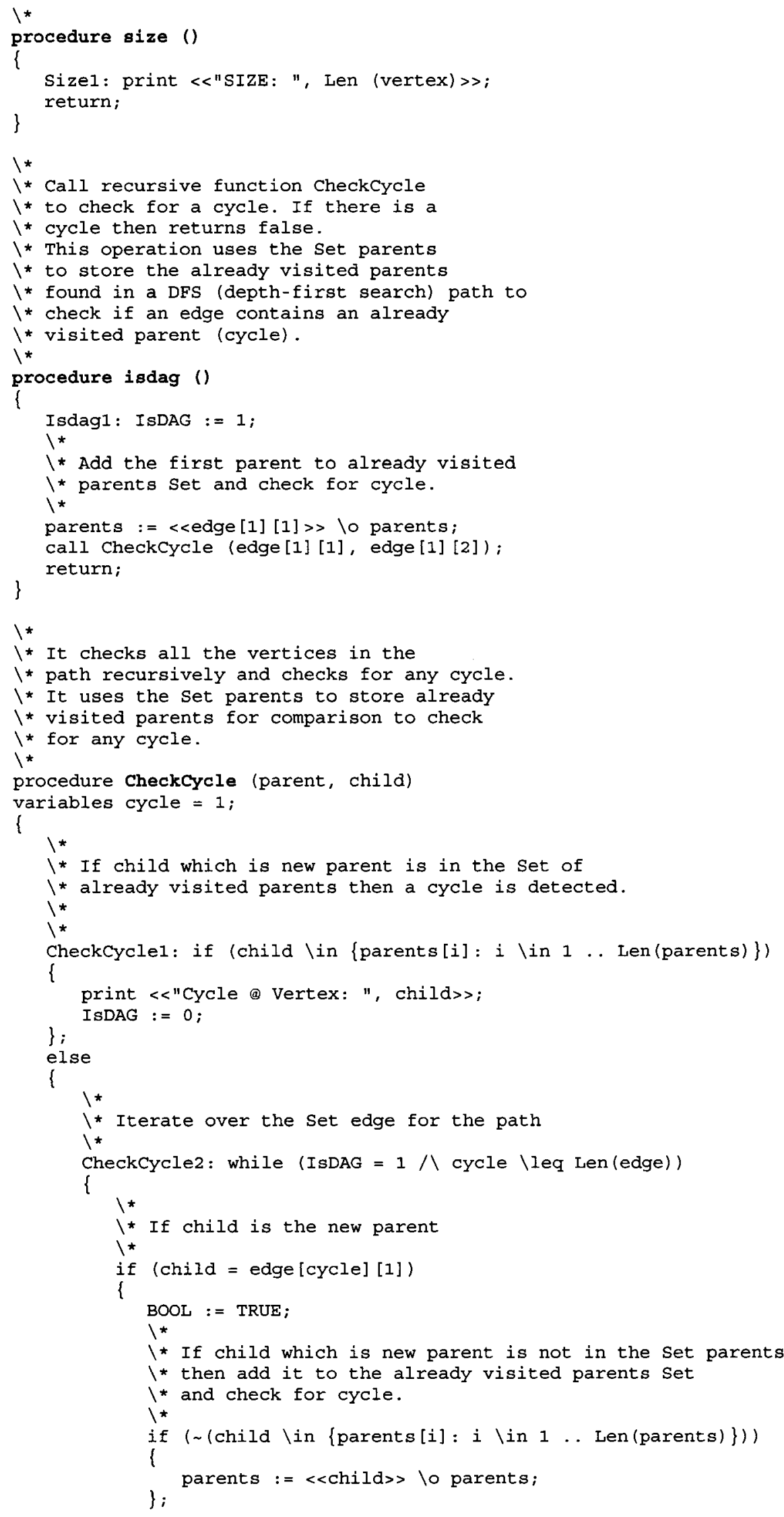




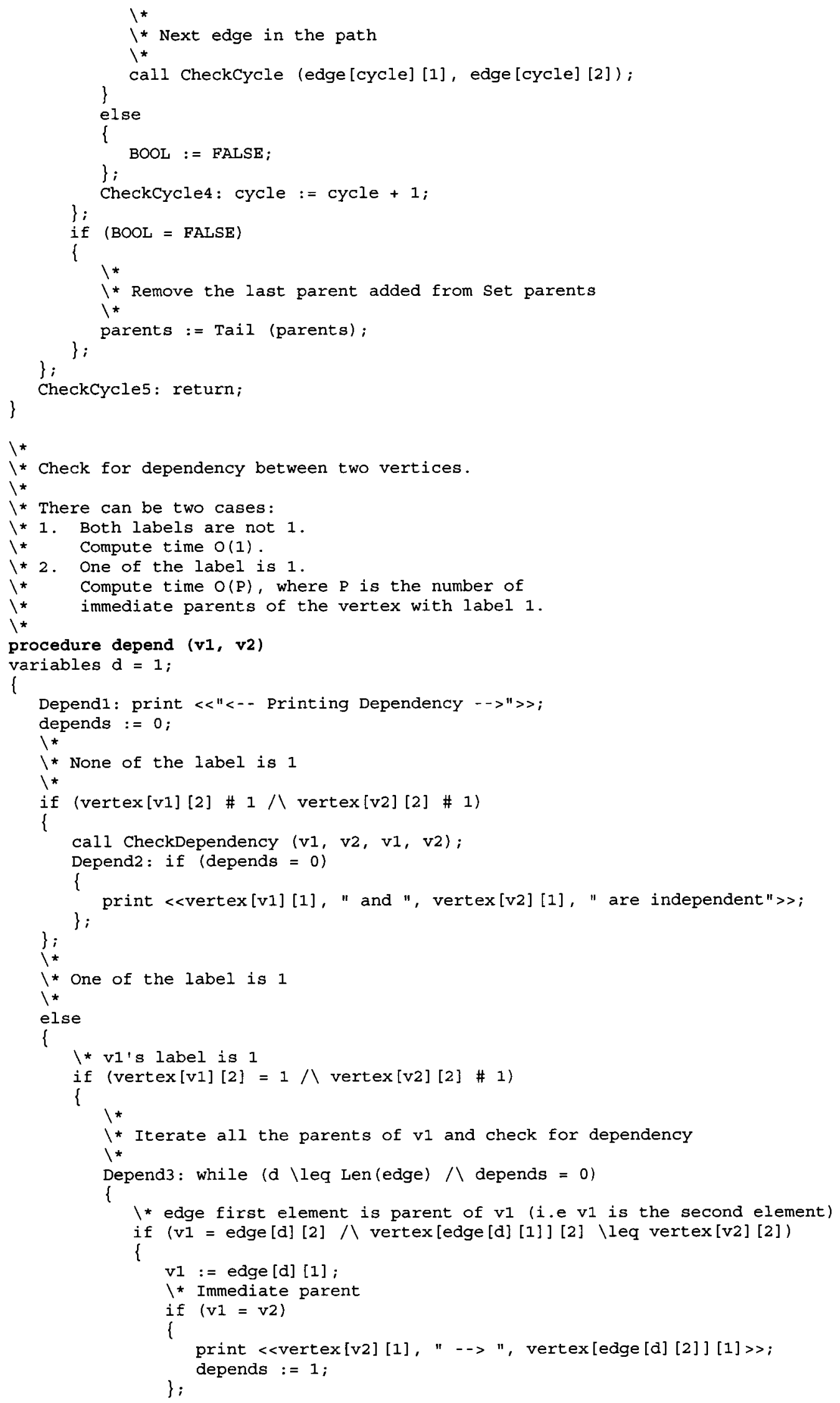




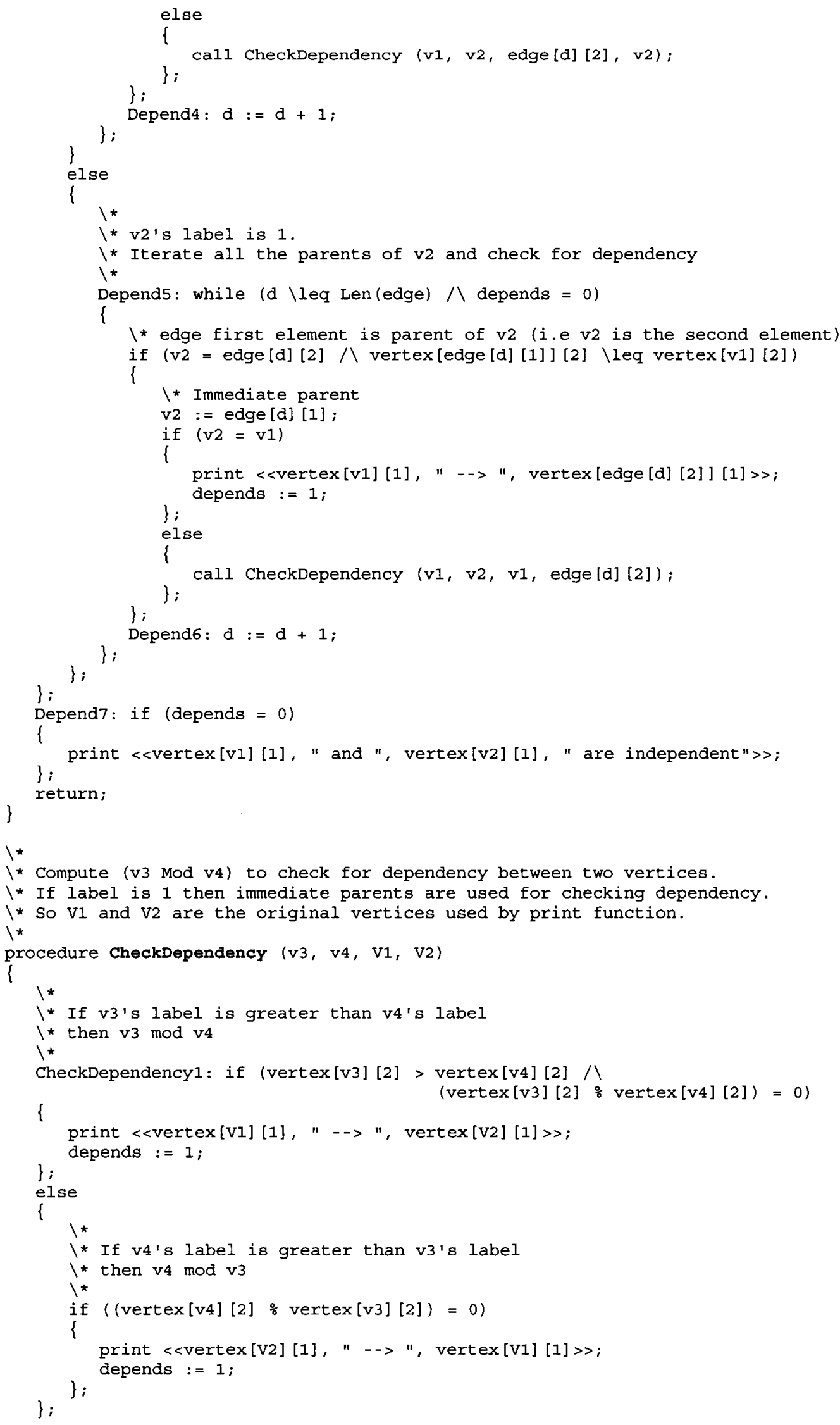




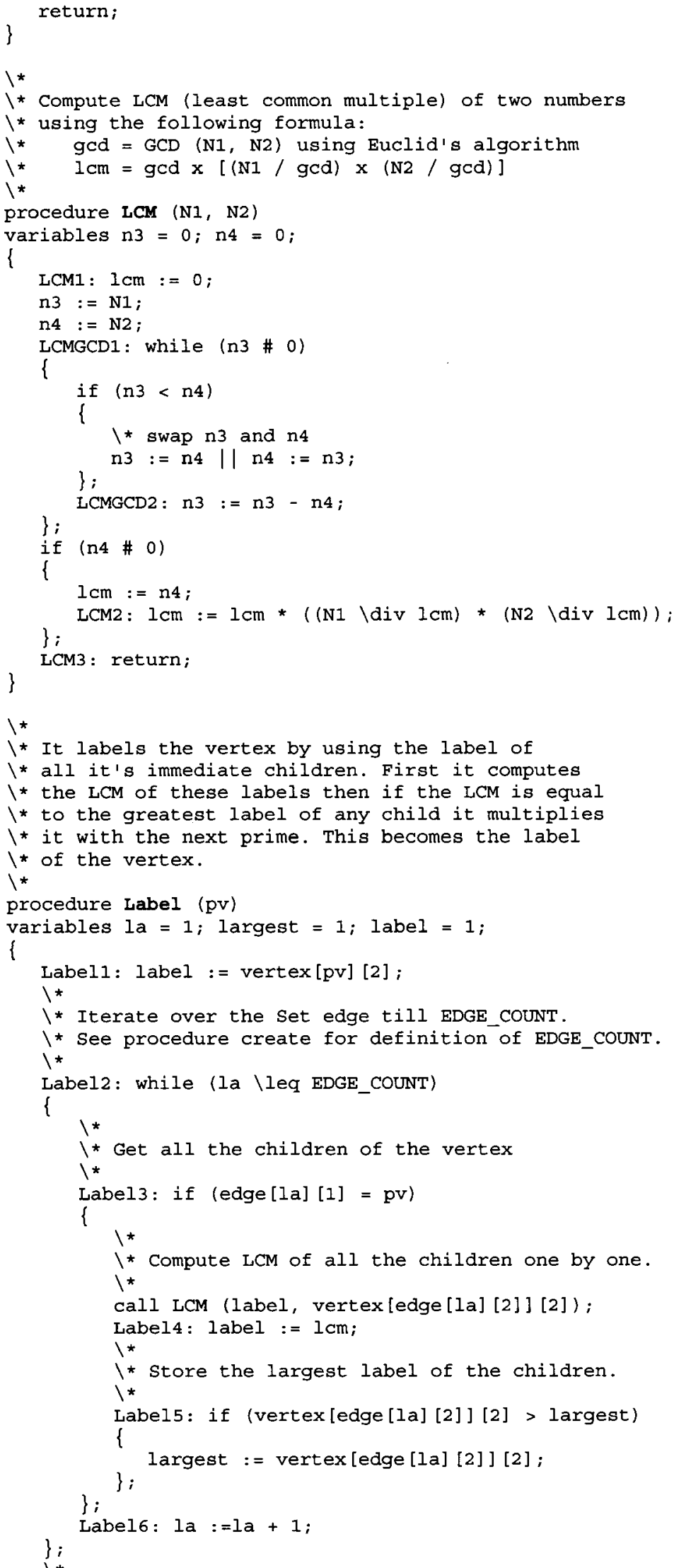




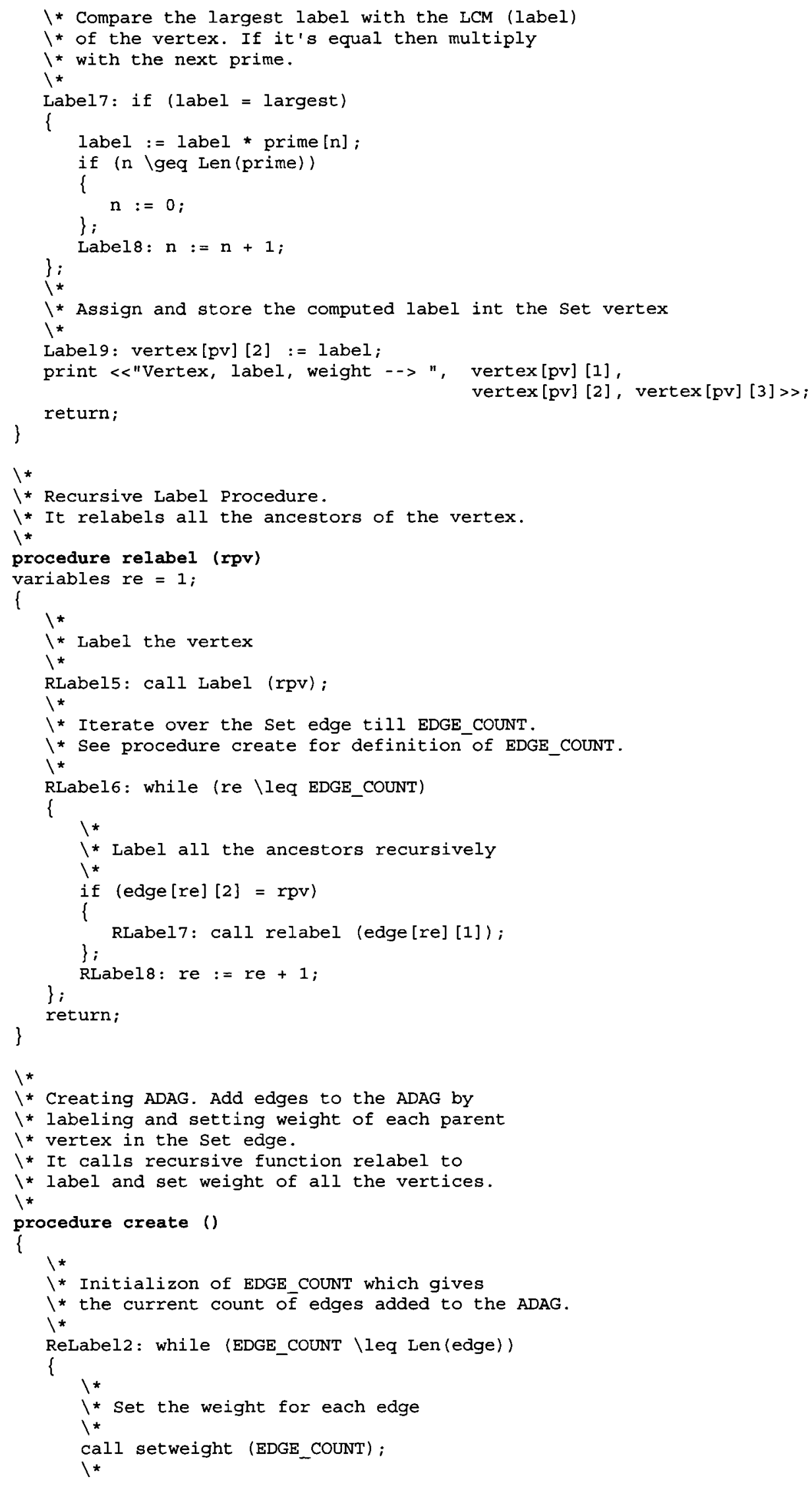




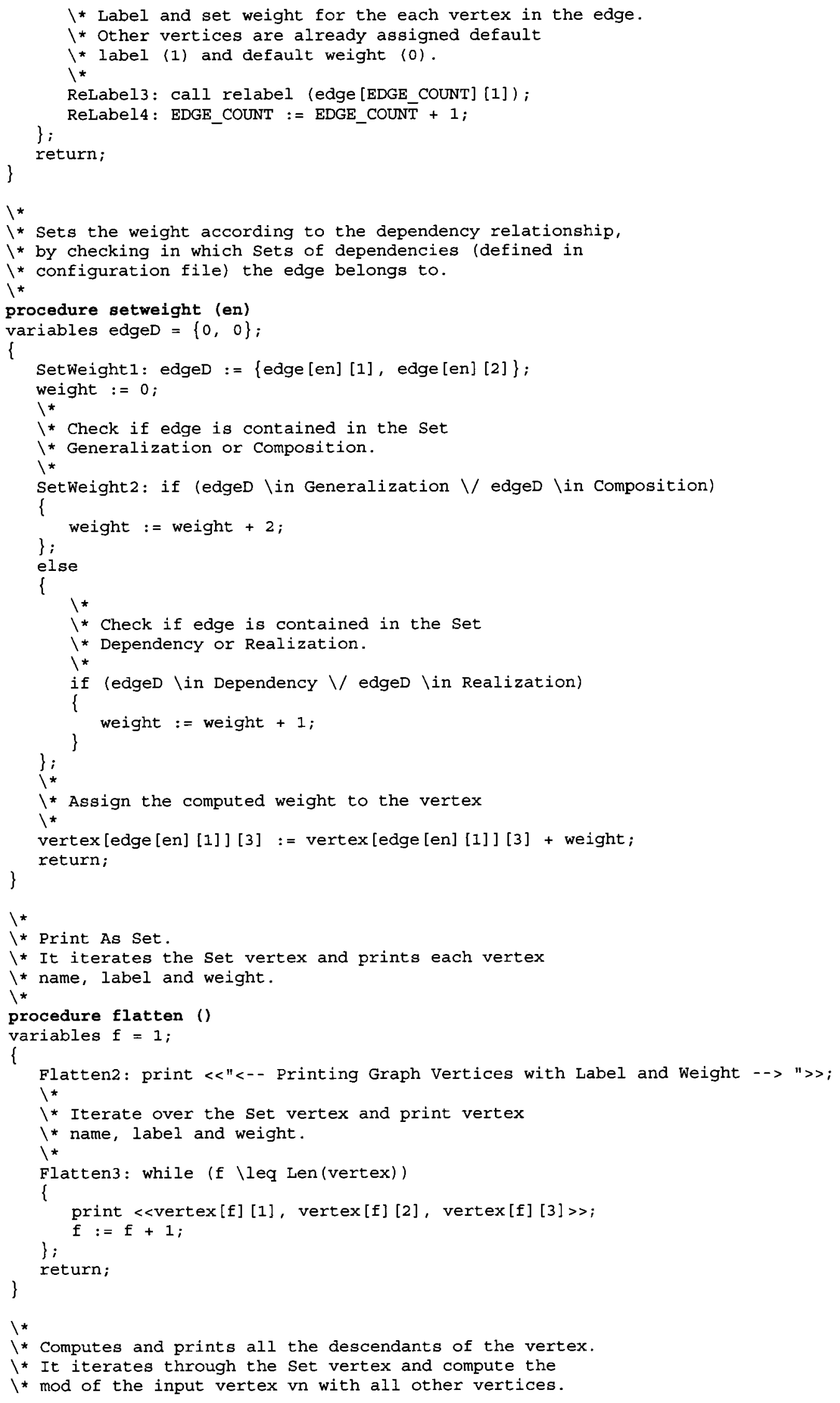




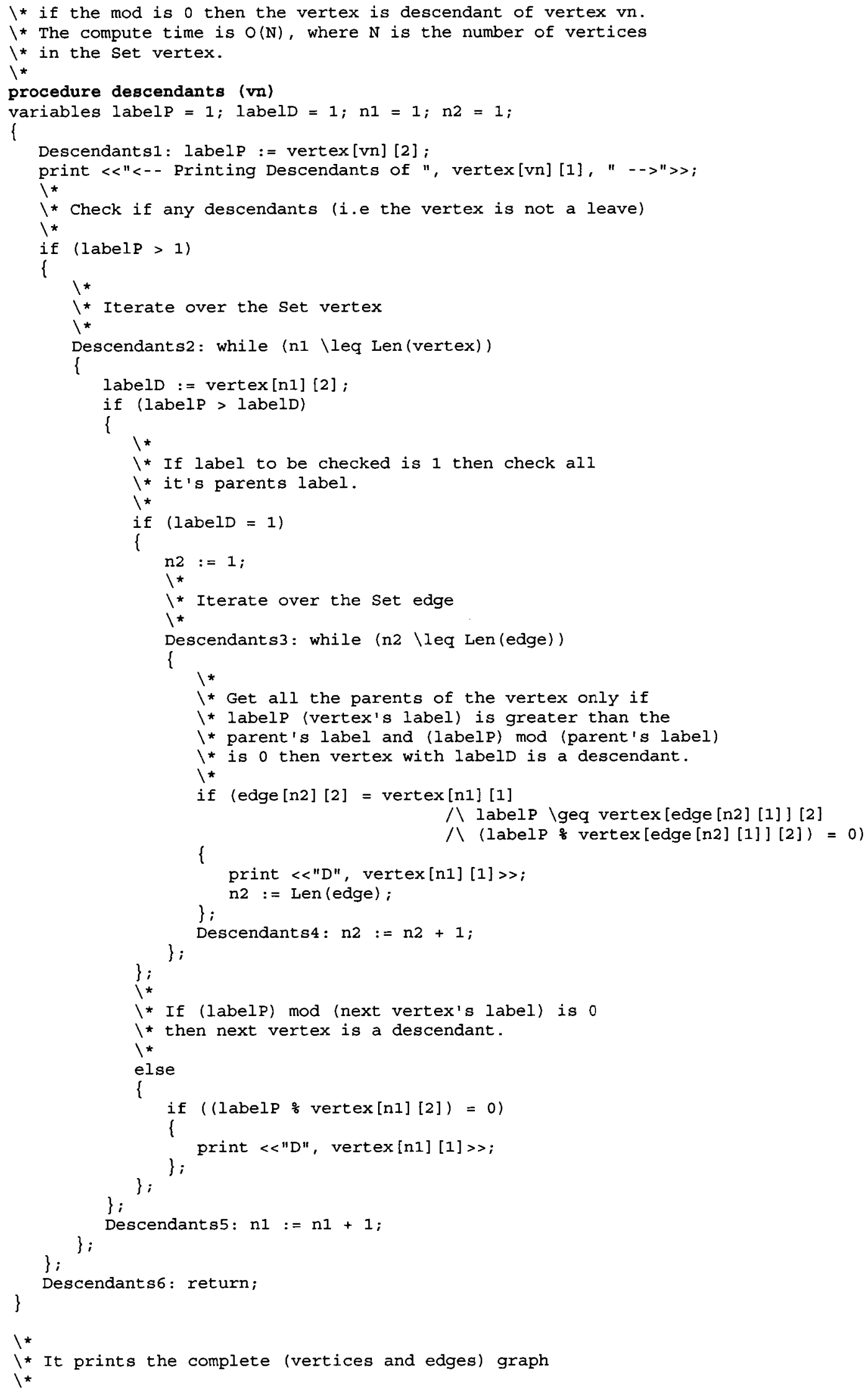




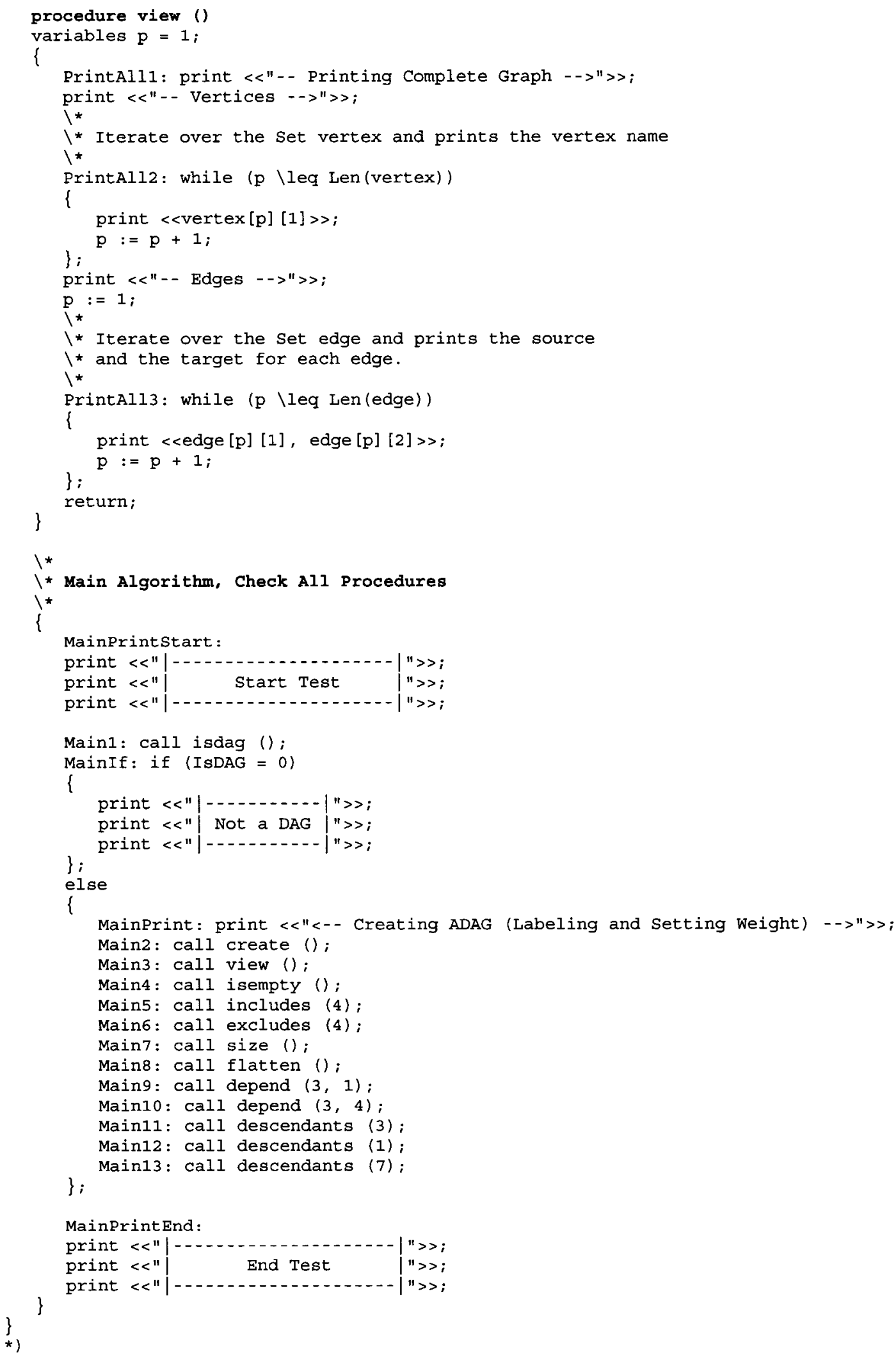




\section{Configuration File}

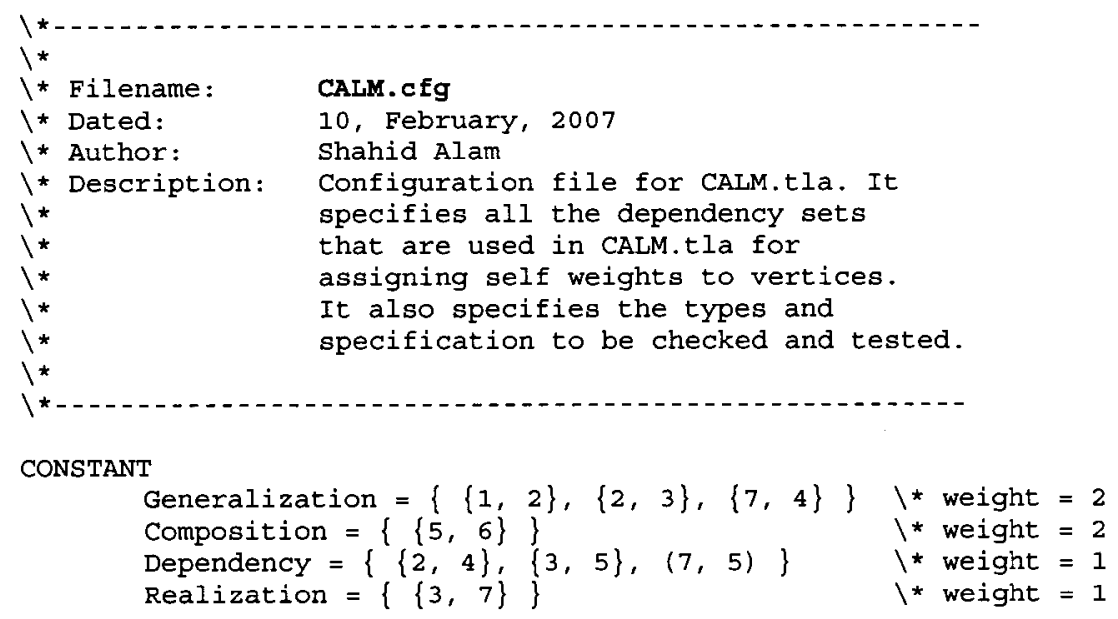

INVARIANT TYpeInvariant

SPECIFICATION Spec

\section{TLC Run for Model Checking}

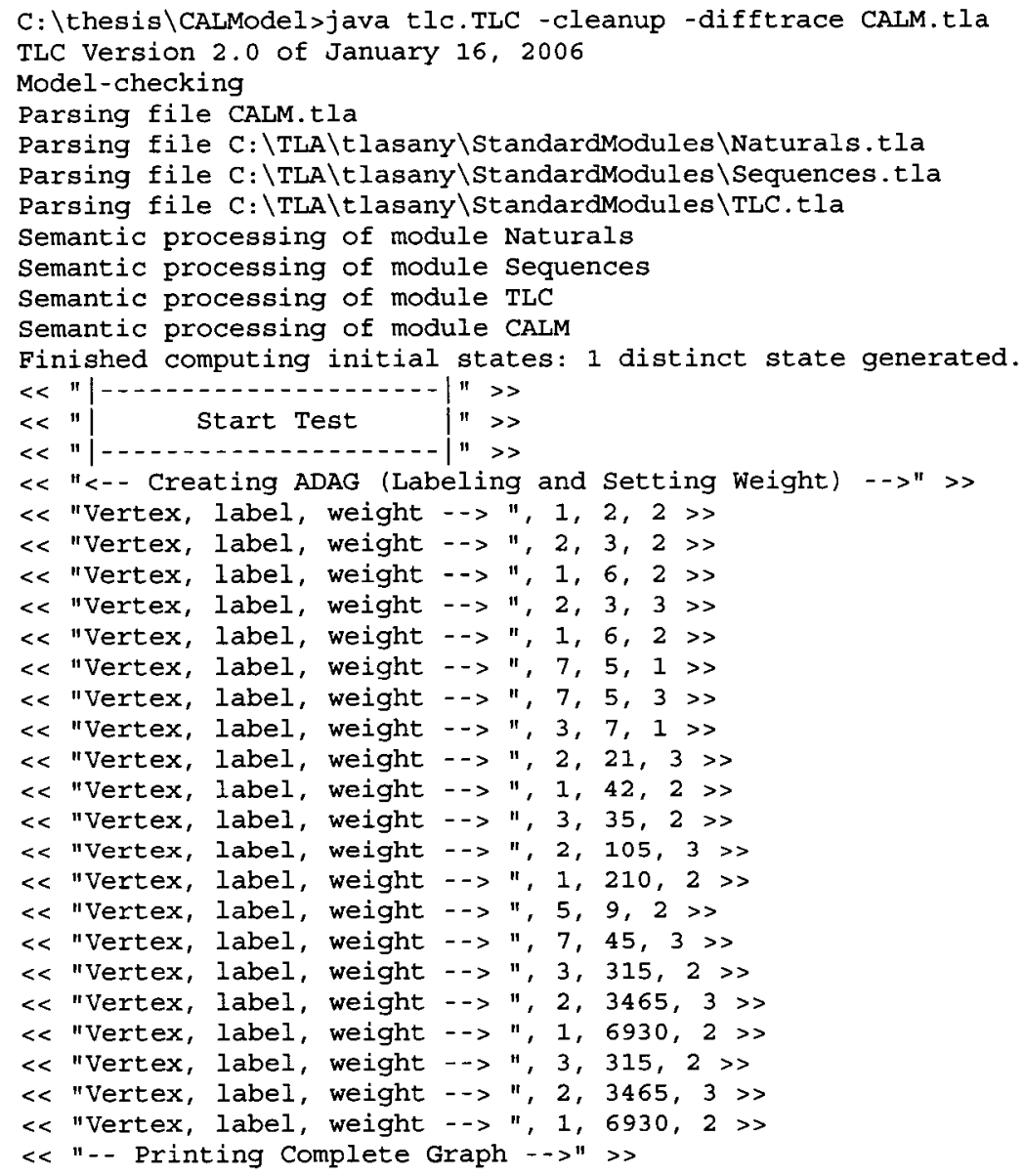




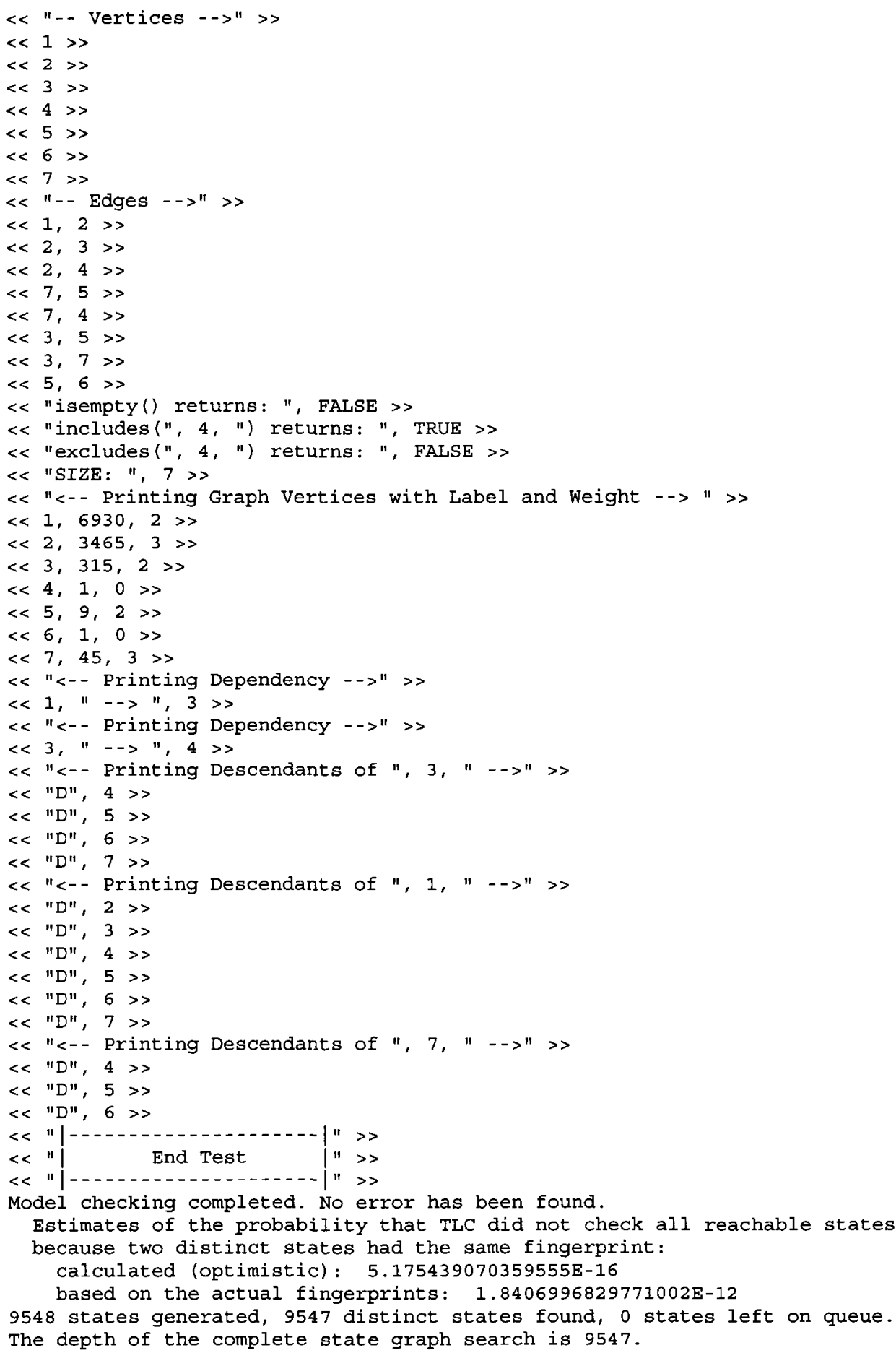




\section{UML Class Diagram in XMI for the Case Study}

This appendix lists the UML Class Diagram in XMI for the case study described in Chapter 6. An open source UML CASE tool StarUML [60] is used to generate the XMI from the UML Class Diagram of the CAL parser. We have not listed the complete XMI. Only the classes and their relationships are shown.

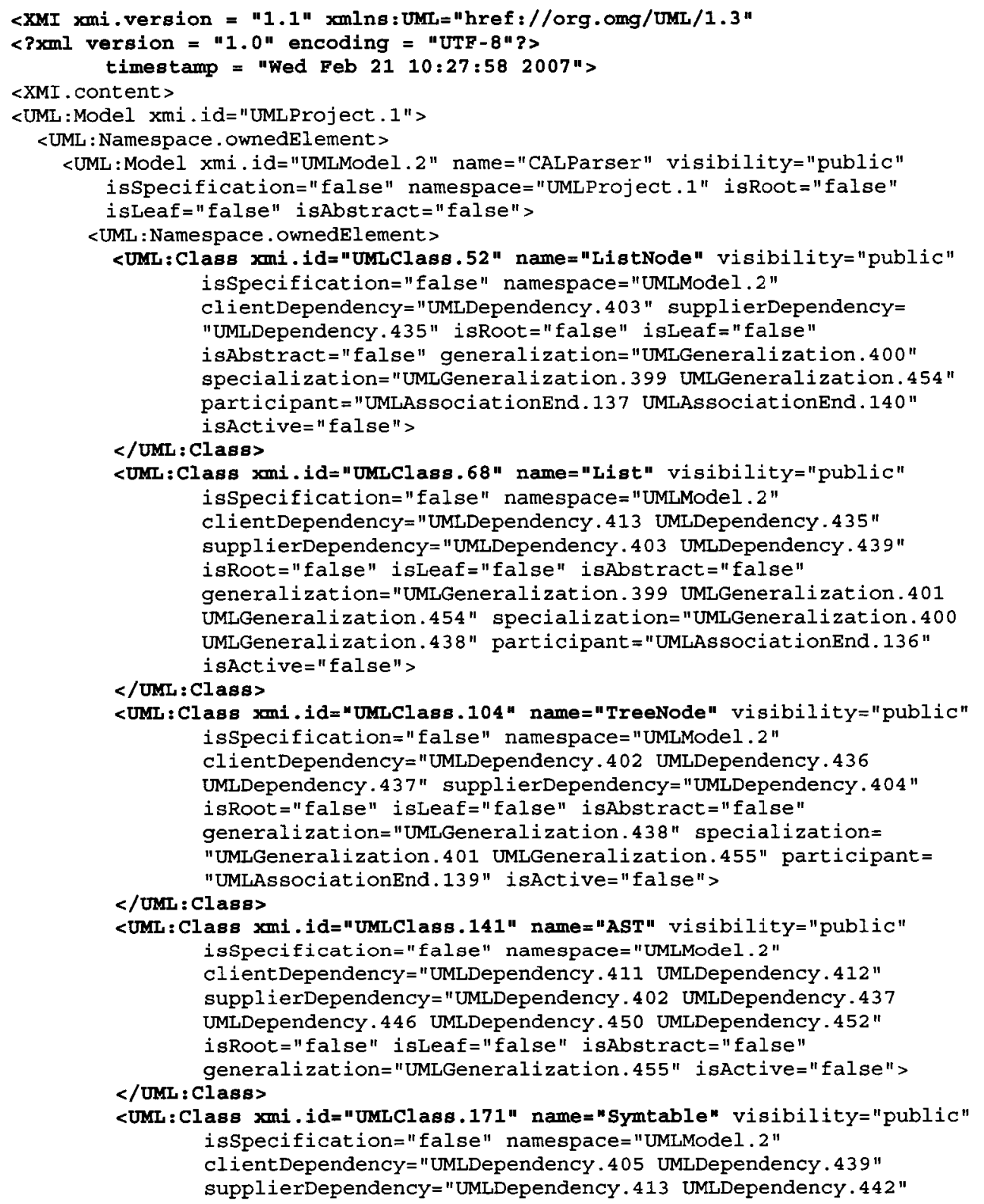




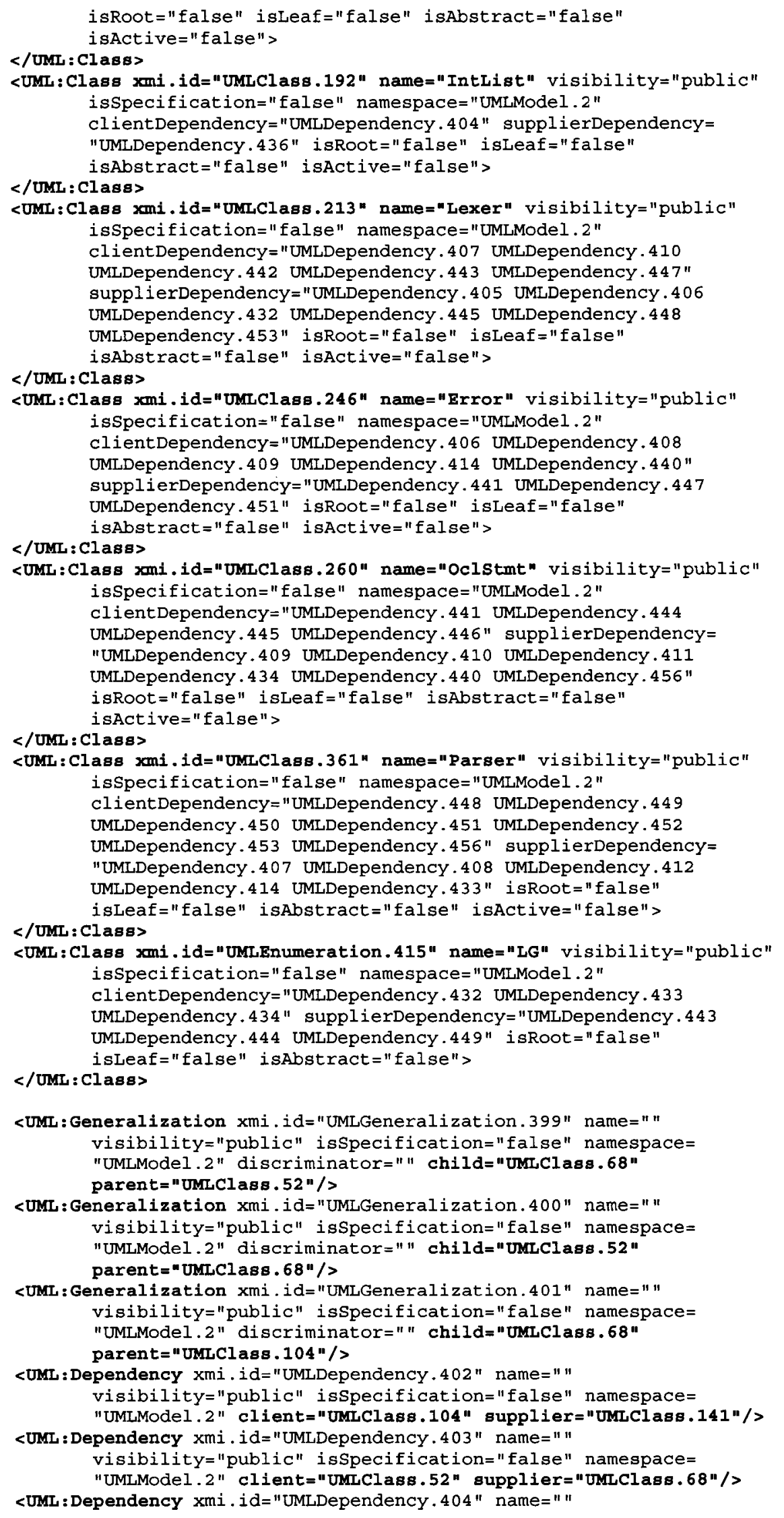




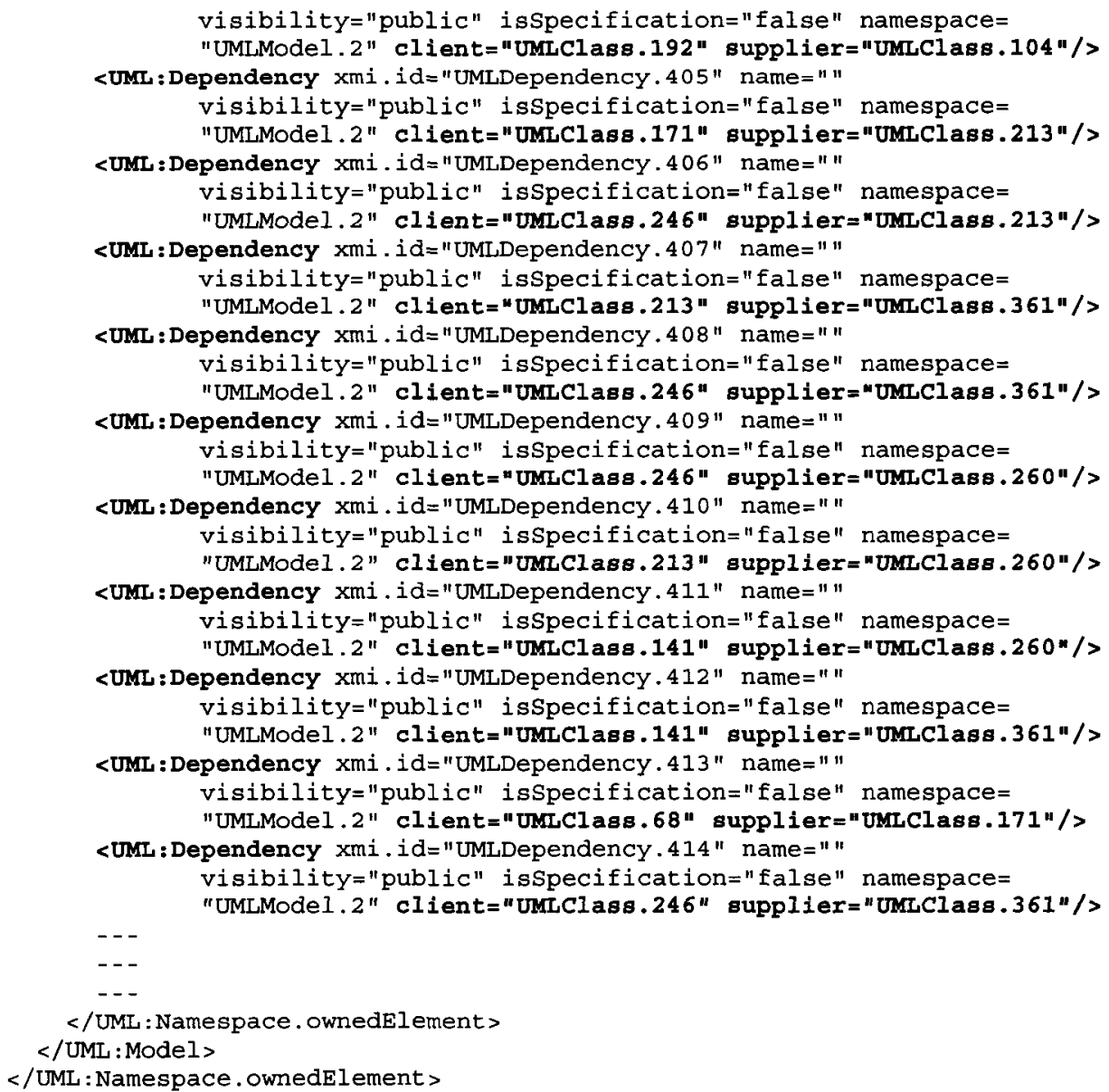




\section{References}

[1] International Standard Organization document reference ISO/IEC 14977:1996(E), "Information Technology - Syntactic Metalanguage - Extended Backus-Naur Form," 1996.

[2] Object Management Group (OMG) Inc, "OCL 2.0 Specification Version 2.0," OMG (C) 2005, document ptc/2005-06-06.

[3] Alfred V. A., Ravi S, and Jefrey D. U., "Compilers Principles, Techniques and Tools," Addison-Wesley $\odot, 1986$.

[4] Madhavji, N. H., Fernandez-Ramil, J., and Perry, D, "Software Evolution and Feedback: Theory and Practice," John Wiley \& Sons $\odot 2006$.

[5] Object Management Group (OMG) Inc, "Unified Modeling Language: Superstructure, version 2.0," OMG @ 2005, document formal/05-07-04.

[6] Alfred V. A., Jeffrey D. U., "The Theory of Parsing, Translation, and Compiling, Vol.1, Parsing”, (C) Prentice-Hall, Englewood Cliffs, New Jersey, 1972

[7] Robert A., Shawn B., "Software Change Impact Analysis", June 1996, (O Wiley-IEEE Computer Society Press.

[8] Thomas H. Cormen, Charles E. Leiserson, Ronald L. Rivest, and Clifford S., "Introduction to Algorithms, Second Edition," MIT Press and McGraw-Hill @ 2001.

[9] Object Management Group (OMG) Inc, "Semantics of a Foundational Subset for Executable UML Models", OMG ( $2005 / 2006$, RFP, [Online-June 7, 2007], Available: http://www.omg.org/docs/ad/05-04-02.pdf.

[10] Craig L., Victor R. B., "Iterative and Incremental Development: A Brief History," Computer, vol.36, no.6, pp. 47-56, Jun., 2003.

[11] Object Management Group (OMG) Architecture Board MDA Drafting Team, "Model Driven Architecture Guide Version 1.0", OMG ○ 2003, [Online-June 7, 2007], Available: http://www.omg.org/docs/omg/03-05-01.pdf.

[12] Sunye, G. (IRISA, Rennes, France), Le Guennec, A., Jezequel, J. M., "Using UML action semantics for model execution and transformation", Information Systems, v 27, Elsevier, UK @ Sept. 2002, p 445-57.

[13] Opdyke W. F., "Refactoring: A Program Restructuring Aid in Designing ObjectOriented Application Frameworks", PhD Thesis, University of Illinois at UrbanaChampaign, 1992. 
[14] Zhang J., Lin Y., and Gray J., "Generic and Domain-Specific Model Refactoring Using a Model Transformation Engine", Chapter 9 in Model-Driven Software Development, S. Beydeda, M. Book, and V. Gruhn, Editors, Springer-Verlag, Heidelberg, Germany, pp. 199-218, 2005.

[15] Łukasz D, Ludwik K, "An approach to refactoring of executable UML models," Proceedings of the 2006 ACM symposium on Applied computing, pp 1273 - 1279, 2006.

[16] Stephen J. Mellor, Marc J. Balcer, Stephen M, Marc B, "Executable UML: A Foundation for Model Driven Architecture", Addison-Wesley Professional, 1st edition, (C) 2002.

[17] Von K. A. (Fraunhofer IESE, Kaiserslautern, Germany), Grund M., "QuaTrace: a tool environment for (semi-) automatic impact analysis based on traces", Proceedings International Conference on Software Maintenance ICSM 2003, pp 246-55 IEEE Computer Society @ 2003.

[18] Ubayashi, N. (Kyushu Inst. of Technol., Japan), Tamai, T., Sano, S., Maeno, Y., Murakami, S., "Model compiler construction based on aspect-oriented mechanisms", Generative Programming and Component Engineering, 4th International Conference, GPCE 2005, Proceedings (Lecture Notes in Computer Science Vol.3676), 2005, pp 109124 Springer-Verlag $(2005$.

[19] Ubayashi, N. (Kyushu Inst. of Technol., Fukuoka, Japan), Tamai, T., Sano, S., Maeno, Y., Murakami, S., "Model evolution with aspect-oriented mechanisms", Proceedings, Eighth International Workshop on Principles of Software Evolution, 2005, pp 187-194, IEEE Computer Society (C 2005.

[20] Gray, J. (Dept. of Comput. \& Inf. Sci., Alabama Univ., Birmingham, AL, USA), Lin, Y., Zhang, J., "Automating change evolution in model-driven engineering", Computer, v 39, n 2, Feb. 2006, pp 51-58, IEEE Computer Society (C) 2006.

[21] Jeffrey G., "Aspect Oriented Domain Specific Modeling: A Generative Approach Using a Metaweaver Framework" PhD Thesis; Computer Science Department, Vanderbilt University (C) 2002.

[22] Briand L. C., Labiche Y., O'Sullivan L., Sówka M. M., "Automated impact analysis of UML models", Journal of Systems and Software, Volume 79, Issue 3, March 2006, Published by Elsevier Science Inc $\mathbb{C} 2006$.

[23] Briand L. C., Labiche Y., O'Sullivan L., "Impact Analysis and Change Management of UML Models," ICSM 2003, pp. 256, 19th IEEE International Conference on Software Maintenance, 2003.

[24] Accelerated Technology, Embedded Systems Division of Mentor Graphics Corporation, "Object Action Language Manual", SAP\# 222021, Mentor Graphics Corporation (c) 2004. 
[25] Ian W., Adrain K., Mike C., Chas W. and Chris R., "ASL Language Reference Level 2.5, Manual Revision C," Kennedy Carter Limited (C 2001.

[26] Pathfinder Solutions, "Platform Independent Action Language Version 2.2 Reference Manual", Pathfinder Solutions LLC (C 2004.

[27] OMG document, "Precise Action Semantics for UML", [Online-June 7, 2007], Available: http://www.omg.org/cgi-bin/doc?ptc/02-01-09, OMG Inc @ 2002.

[28] Tom M., and Theo D, "Automating support for software evolution in UML," Automated Software Engineering Journal, 7(1): pp 39--59, February 2000.

[29] Suguru S., Naoyasu U., Hideaki S., and Tetsuo T.: “An Extensible Contract Verifier for AspectJ," 2nd Asian Workshop on Aspect-Oriented Software Development (AOAsia 2) (Workshop at ASE 2006), pp.35-40 (2006).

[30] Kiczales G., Hilsdale E., Hugunin J., Kersten M., Palm J., and Griswold W. G. 2001. "An Overview of AspectJ," In Proceedings of the 15th European Conference on ObjectOriented Programming (June 18 - 22, 2001). J. L. Knudsen, Ed. Lecture Notes in Computer Science, vol. 2072. Springer-Verlag, London, 327-353.

[31] Briand, L. C., Labiche, Y., O'Sullivan, L., 2003. "Impact Analysis and Change Management of UML Models," SCE-03-01, Carleton University, [Online-June 7, 2007], Available: http://squall.sce.carleton.ca/pubs/tech_report/TR_SCE-03-01.pdf.

[32] Telelogic Rhapsody [Online-June 7, 2007], Available: http://modeling.telelogic.com/products/rhapsody/.

[33] IBM Rational Rose [Online-June 7, 2007], Available: http://www306.ibm.com/software/rational/.

[34] IBM XDE [Online-June 7, 2007], Available: http://www306.ibm.com/software/awdtools/developer/rosexde/.

[35] Telelogic Rhapsody Gateway technical brochure [Online-June 7, 2007], Available: http://modeling.telelogic.com/products/rhapsody/add-on/gateway.cfm.

[36] Santoro N. and Khatib R., "Labelling and implicit routing in networks", Journal of Computing., 28(1): pp. 5-8, 1985.

[37] Gavoille, C. and Peleg, D., 2003, "Compact and localized distributed data structures”. Journal of Distributed Computing, 16, 2-3 (September, 2003).

[38] Vassilis C., Gregory K., Dimitris P., Michel S., Sotirios T., “Optimizing taxonomic semantic web queries using labeling schemes", Journal of Web Semantics, Volume 1(2): pp. 207-228 (2004).

[39] Cohen, E., Halperin, E., Kaplan, H., and Zwick, "Reachability and Distance Queries via 2-Hop Labels,” SIAM Journal of Computing, 32, 5 (May. 2003), pp. 1338-1355. 
[40] Gang W., Kuo Z., Can L., Juan-Zi L., “Adapting Prime Number Labeling Scheme for Directed Acyclic Graphs", Lecture Notes in Computer Science Vol 3882, pp 787-796, Springer-Verlag @ 2006.

[41] Xiaodong W., Mong L. L., Wynne H., "A Prime Number Labeling Scheme for Dynamic Ordered XML Trees," ICDE'04, pp. 66, 20th International Conference on Data Engineering, 2004.

[42] Hongzhi W., Wei W., Xuemin L., Jianzhong L., "Labeling Scheme and Structural Joins for Graph-Structured XML Data," Lecture Notes in Computer Science Vol 3399, pp 277-288, Springer-Verlag (C) 2005.

[43] Li, C., Ling, T. W., Lu, J., and Yu, T. 2005. "On reducing redundancy and improving efficiency of XML labeling schemes", In Proceedings of the 14th ACM International Conference on information and Knowledge Management (Bremen, Germany, October 31 - November 05, 2005), CIKM '05.

[44] Mens, T., Wermelinger, M., Ducasse, S., Demeyer, S., Hirschfeld, R., Jazayeri, M., "Challenges in software evolution," Principles of Software Evolution, Eighth International Workshop on , pp. 13- 22, 5-6 Sept. 2005.

[45] Lindvall, M. and Sandahl, K, 1998, "How well do experienced software developers predict software change?" Journal of Systems and Software, 43, 1 (Oct. 1998), 19-27.

[46] Martin, R. C., 2003, "Agile Software Development: Principles, Patterns, and Practices,” Prentice Hall PTR.

[47] Mellor S. J., Kendall S., Uhl A., and Weise D., "MDA Distilled," Addison Wesley Longman Publishing Co., Inc, 2004.

[48] JGraph, Java Graph Visualization and Layout, [Online-June 7, 2007], Available: http://www.jgraph.com.

[49] OpenGL, Open Graphics Library, [Online-June 7, 2007], Available: http://www.opengl.org.

[50] Lamport, L. 2003, "Specifying Systems: the Tla+ Language and Tools for Hardware and Software Engineers," Addison-Wesley Longman Publishing Co., Inc, 2003.

[51] Elrad T., Filman R. E., and Bader A, 2001, "Aspect-oriented programming: Introduction," Communication of the ACM, Vol. 44, Issue. 10 (Oct. 2001), pp: 29-32.

[52] Jazayeri, M., 2005, "Species evolve, individuals age," In Proceedings of the Eighth international Workshop on Principles of Software Evolution (September 05 - 06, 2005). IWPSE, IEEE Computer Society, Washington, DC, 3-12.

[53] pluscal user manual, C-syntax version, by Lamport L., [Online-June 7, 2007], Available: http://research.microsoft.com/users/lamport/tla/c-manual.pdf. 
[54] SWT, Standard Widget Toolkit, [Online-June 7, 2007], Available: http://www.eclipse.org/swt.

[55] JNI, Java Native Interface, [Online-June 7, 2007], Available: http://java.sun.com/j2se/1.4.2/docs/guide/jni.

[56] Java AWT, Abstract Window Toolkit, [Online-June 7, 2007], Available: http://java.sun.com/j2se/1.4.2/docs/guide/awt/index.html.

[57] API documentation of CAL Parser, [Online-June 7, 2007], Available: http://chat.carleton.ca/ salam3/thesis/cal/docs/html/index.html.

[58] API documentation of VCAL, [Online-June 7, 2007], Available: http://chat.carleton.ca/ salam3/thesis/cal/docs/java/html/index.html.

[59] VCAL online user manual, [Online-June 7, 2007], Available: http://chat.carleton.ca/ salam3/thesis/cal/html/help.html

[60] StarUML, Open source UML Platform for Win32, [Online-June 7, 2007], Available: http://staruml.sourceforge.net/en/.

[61] RISE, Research Institue for Software Evolution, University of Durham, England, [Online-June 7, 2007], Available: http://www.dur.ac.uk/RISE.

[62] VCAL package download, [Online-June 7, 2007], Available: http://chat.carleton.ca/ salam3/thesis/cal/runvcal.zip.

[63] VCAL online demo, [Online-June 7, 2007], Available: http://chat.carleton.ca/ salam3/thesis/cal/demo/VCALDemo.html.

[64] CAL, Constraints with action language, [Online-June 7, 2007], Available: http://chat.carleton.ca/ salam3/thesis/cal/cal.html. 